Acta Crystallographica Section B

Structural

Science

ISSN 0108-7681

\title{
Structure of ureido-balhimycin
}

G. M. Sheldrick, E. Paulus, L. Vértesy and F. Hahn

This electronic document was scanned from an archival copy of material deposited to accompany a paper published in an IUCr journal. In many cases the only accessible copy was a microfilm of a poor-quality original. 


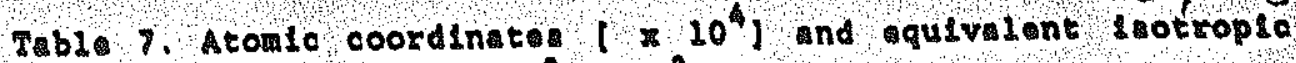
displacoment peraneters $\left(A^{2} \times 10^{3}\right)$ for $1, U(0 q)$ lo desined as one third of the trace of the orthogonalised $v_{1}$ t tonsor.

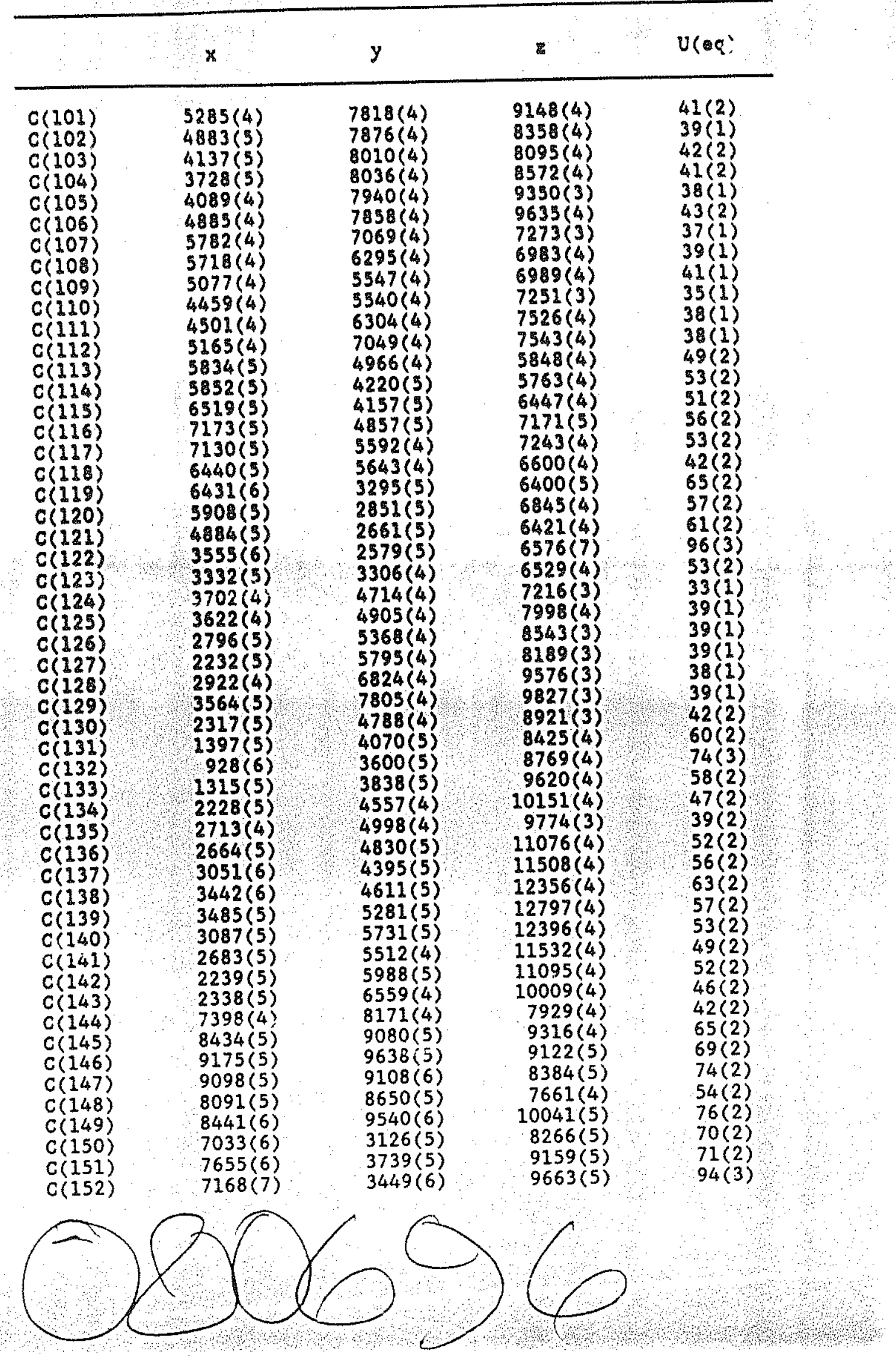




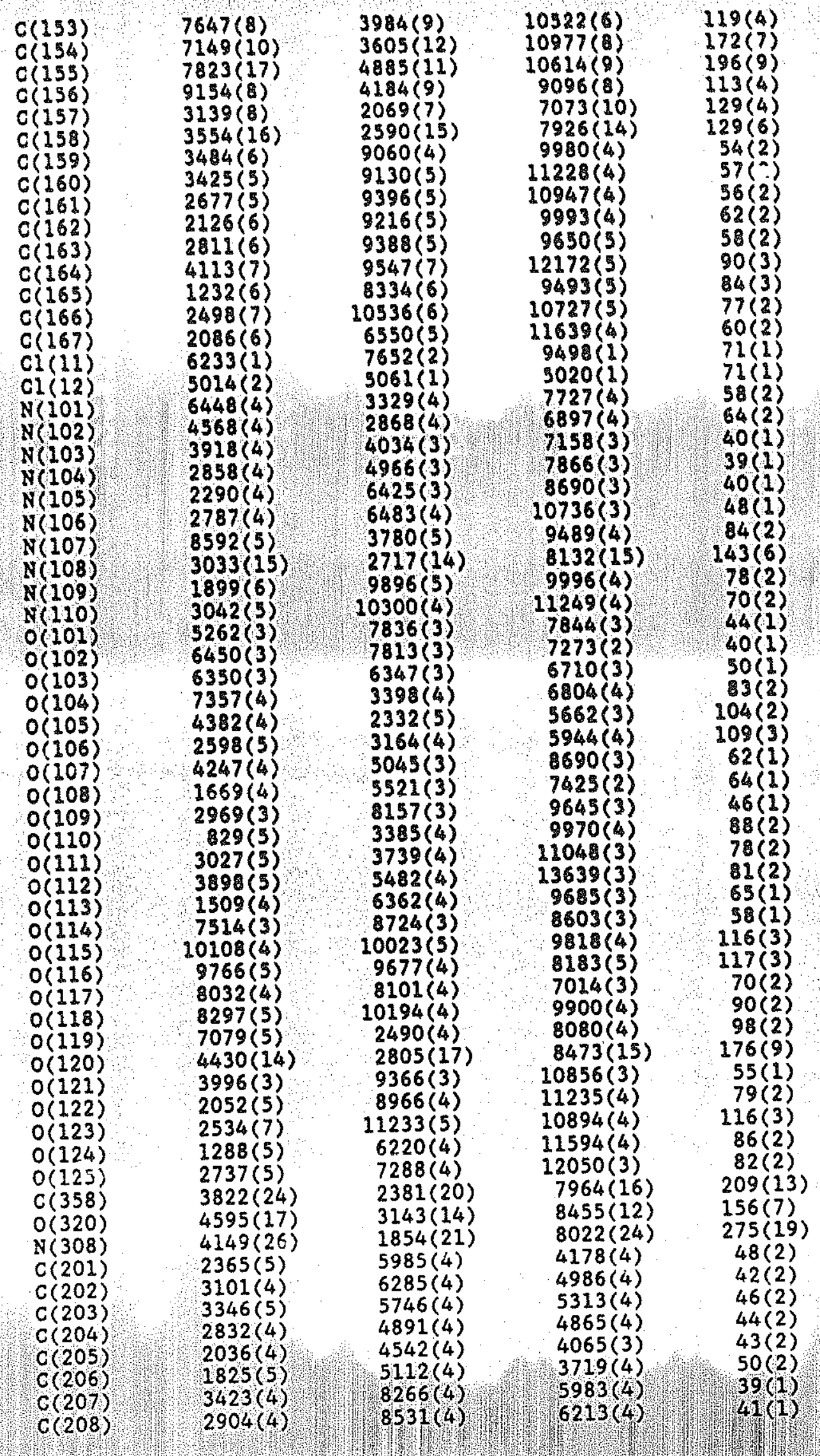




\begin{tabular}{|c|c|c|c|c|}
\hline $\begin{array}{l}c(209) \\
c(210) \\
c(211) \\
c(212) \\
c(213) \\
c(214) \\
c(215) \\
c(216) \\
c(217) \\
c(218) \\
c(219) \\
c(220) \\
c(221) \\
c(222) \\
c(223) \\
c(224) \\
c(225) \\
c(226) \\
c(227) \\
c(228) \\
c(229) \\
c(230) \\
c(231) \\
c(232) \\
c(233) \\
c(234) \\
c(235) \\
c(236) \\
c(237) \\
c(238) \\
c(239) \\
c(240) \\
c(241) \\
c(242) \\
c(243) \\
c(244) \\
c(245) \\
c(246) \\
c(247) \\
c(248) \\
c(249) \\
c(250) \\
c(251) \\
c(252) \\
c(253) \\
c(234) \\
c(255) \\
c(353) \\
c(354) \\
c(355) \\
c(256) \\
c(257) \\
c(258) \\
c(259) \\
c(260) \\
c(261) \\
c(262) \\
c(263) \\
c(264) \\
c(265) \\
c(266) \\
c(267) \\
c 1(21)\end{array}$ & $\begin{array}{l}2120(5) \\
1865(4) \\
2363(4) \\
3108(5) \\
2560(5) \\
1847(5) \\
1121(5) \\
1156(6) \\
1870(5) \\
2545(5) \\
263(6) \\
.591(5) \\
-299(5) \\
-599(6) \\
243(6) \\
1041(4) \\
442(4) \\
225(4) \\
949(4) \\
588(5) \\
1360(5) \\
-749(5) \\
-936(6) \\
-1782(7) \\
-2407(6) \\
2240(5) \\
-1415(4) \\
-2897(5) \\
-3735(5) \\
-4338(5) \\
-4118(5) \\
-3304(5) \\
-2710(5) \\
-1874(5) \\
-2111(5) \\
5089(4) \\
6083(5) \\
6880(5) \\
6808(5) \\
5827(5) \\
6044(6) \\
-1785(6) \\
-2074(7) \\
-2990(14) \\
-3288(19) \\
-3483(18) \\
-4105(25) \\
-3263(22) \\
-2610(29) \\
-4222(24) \\
-1470(13) \\
-1550(7) \\
-1778(9) \\
2472(5) \\
1369(6) \\
1842(5) \\
2444(6) \\
3019(5) \\
963(7) \\
1872(7) \\
3201(7) \\
1951(7) \\
2104(2)\end{array}$ & $\begin{array}{l}7970(4) \\
7115(4) \\
6841(4) \\
7400(4) \\
9801(4) \\
9910(5) \\
9842(5) \\
9714(5) \\
9598(5) \\
9606(4) \\
9836(5) \\
8921(5) \\
8412(5) \\
6987(5) \\
6866(5) \\
6491(4) \\
5656(4) \\
4238(4) \\
3983(4) \\
3309(4) \\
3598(4) \\
3489(4) \\
3016(6) \\
2238(7) \\
1891(5) \\
2318(5) \\
3135(4) \\
1960(5) \\
1990(6) \\
1669(6) \\
1345(5) \\
1259(5) \\
1357(5) \\
1403(5) \\
2368(5) \\
9090(4) \\
9845(5) \\
10457(5) \\
10025(5) \\
9653(5) \\
10192(5) \\
8490(6) \\
8129(7) \\
7246(10) \\
6563(14) \\
6583(16) \\
5733(18) \\
6631(18) \\
6354(28) \\
5820(19) \\
9585(10) \\
6081(7) \\
5699(9) \\
3214(5) \\
1971(5) \\
1481(5) \\
1824(5) \\
2810(5) \\
1732(6) \\
1482(6) \\
1415(6) \\
7734(6) \\
6680(2) \\
\end{array}$ & $\begin{array}{l}6255(3) \\
6081(4) \\
5834(4) \\
5752(4) \\
7138(4) \\
7156(5) \\
6432(5) \\
5705(5) \\
5693(4) \\
6409(4) \\
6426(5) \\
6117(4) \\
6645(4) \\
6668(5) \\
6812(4) \\
6177(4) \\
5437(3) \\
4942(4) \\
5284(3) \\
3865(3) \\
3618(4) \\
4608(4) \\
5113(5) \\
4769(5) \\
3926(5) \\
3402(4) \\
3767(4) \\
2481(4) \\
2135(5) \\
1300(5) \\
772(4) \\
1085(4) \\
1952(4) \\
2315(5) \\
3407(4) \\
6608(4) \\
8060(4) \\
7943(5) \\
7167(5) \\
6417(5) \\
8820(5) \\
4672(5) \\
3773(6) \\
3349(9) \\
3558(16) \\
4235(16) \\
2836(20) \\
2660(20) \\
2618(30) \\
2383(23) \\
3707(10) \\
6174(8) \\
5323(10) \\
3578(4) \\
2352(4) \\
2713(4) \\
3671(4) \\
3981(4) \\
1419(4) \\
4092(5) \\
3101(5) \\
1685(5) \\
3739(1) \\
\text { (4) }\end{array}$ & 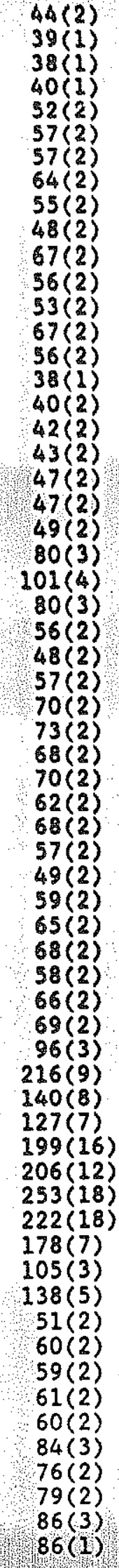 \\
\hline
\end{tabular}




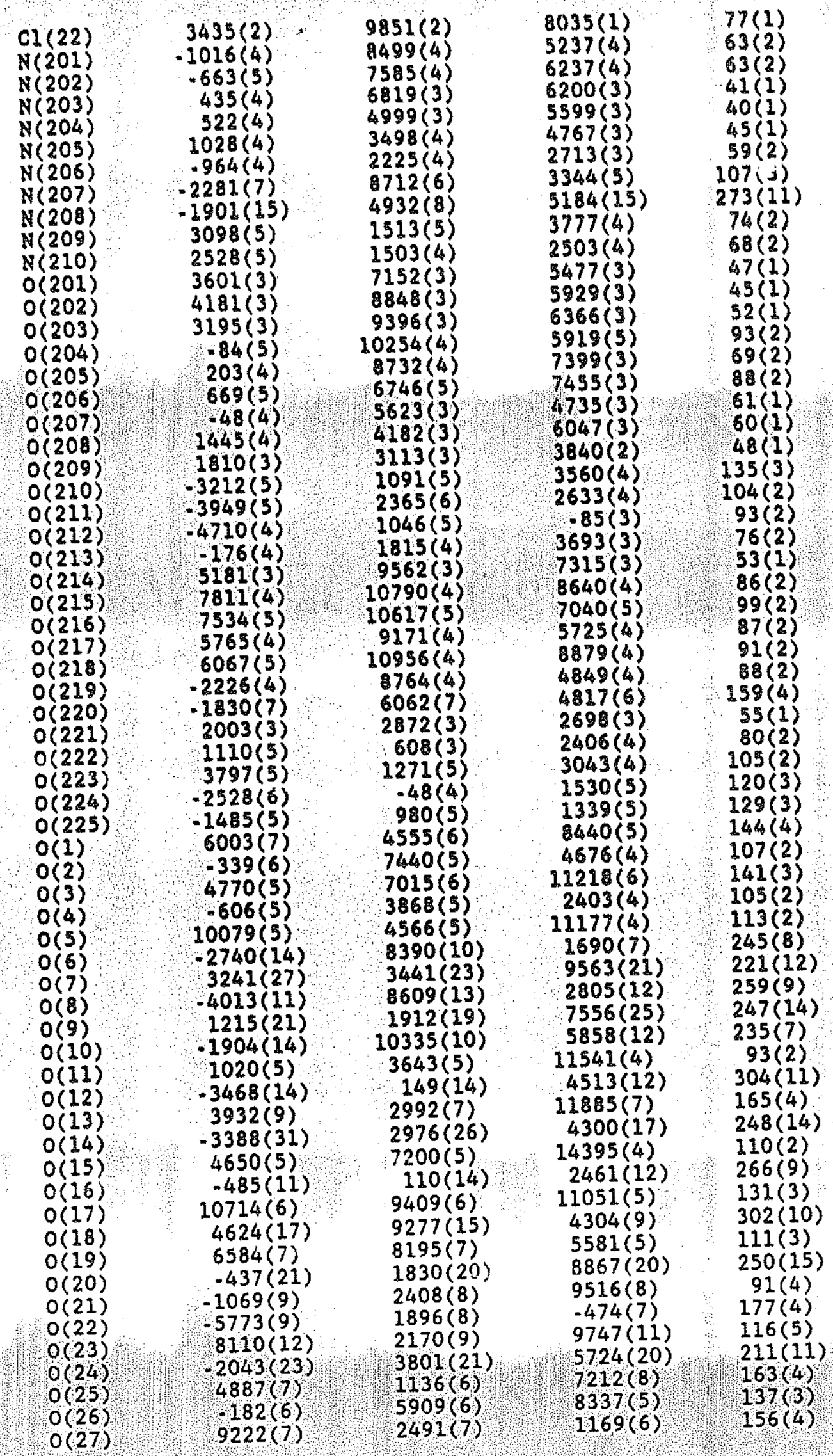




\begin{tabular}{|c|c|c|c|c|}
\hline $\begin{array}{l}0(28) \\
0(29) \\
0(30) \\
0(31) \\
0(32) \\
0(33) \\
0(34) \\
0(35) \\
0(36) \\
0(37) \\
0(38) \\
0(39) \\
0(40) \\
0(41) \\
0(42) \\
0(43) \\
0(44) \\
0(45) \\
0(46) \\
0(47) \\
0(48) \\
0(49) \\
0(50) \\
0(51) \\
0(52) \\
0(53) \\
0(54) \\
0(35) \\
0(56) \\
0(57) \\
0(58) \\
0(59) \\
0(60) \\
0(61) \\
0(62) \\
0(63) \\
0(64) \\
0(65) \\
0(66) \\
0(67) \\
0(68) \\
0(69) \\
0(70) \\
0(71) \\
0(72) \\
0(73) \\
\end{array}$ & $\begin{array}{l}6472(28) \\
7480(24) \\
4597(7) \\
3982(15) \\
9004(17) \\
7358(19) \\
6962(19) \\
2330(15) \\
2162(14) \\
624(10) \\
5239(10) \\
1254(14) \\
564(15) \\
9172(13) \\
5898(7) \\
4232(23) \\
1239(13) \\
11388(29) \\
9474(13) \\
10342(36) \\
10165(18) \\
-2937(28) \\
-4100(43) \\
5372(25) \\
5978(29) \\
-2384(36) \\
4138(25) \\
9037(12) \\
856(11) \\
9652(13) \\
8991(11) \\
2485(20) \\
-4924(20) \\
5240(15) \\
7340(23) \\
9481(27) \\
8147(23) \\
4818(32) \\
8295(39) \\
-1672(45) \\
9375(50) \\
9485(30) \\
6607(41) \\
8686(38) \\
10487(30) \\
9659(19) \\
\end{array}$ & $\begin{array}{c}5964(24) \\
6538(17) \\
8007(7) \\
461(11) \\
4478(13) \\
1913(13) \\
1716(13) \\
8351(10) \\
1089(10) \\
11598(10) \\
5984(11) \\
1769(9) \\
7202(14) \\
5207(9) \\
9496(8) \\
2289(27) \\
2620(15) \\
9338(20) \\
11144(13) \\
11249(31) \\
6522(17) \\
4179(16) \\
3809(34) \\
4301(29) \\
11090(28) \\
4969(38) \\
4094(20) \\
2947(11) \\
7658(11) \\
1925(12) \\
6147(13) \\
8878(15) \\
3431(18) \\
3549(18) \\
6900(20) \\
6496(22) \\
8777(20) \\
10848(31) \\
66664(41) \\
5849(43) \\
7346(39) \\
7802(33) \\
7251(42) \\
8183(36) \\
7051(26) \\
4629(18) \\
\end{array}$ & $\begin{array}{l}9313(25) \\
9121(11) \\
3162(7) \\
5349(12) \\
6769(13) \\
6608(16) \\
6119(14) \\
2696(11) \\
8925(11) \\
5245(8) \\
442(8) \\
9680(8) \\
833(14) \\
3008(6) \\
1776(7) \\
4074(16) \\
6664(13) \\
3152(28) \\
7922(13) \\
8171(28) \\
2403(13) \\
3008(23) \\
2134(42) \\
9483(20) \\
4980(24) \\
3176(33) \\
10175(18) \\
6920(10) \\
4042(9) \\
6768(12) \\
9270(9) \\
4192(13) \\
1026(23) \\
3624(11) \\
884(18) \\
2575(21) \\
3310(19) \\
4393(23) \\
12418(36) \\
3455(42) \\
1679(41) \\
964(31) \\
5373(35) \\
1094(38) \\
2038(27) \\
7227(19) \\
\end{array}$ & $\begin{array}{l}302(21) \\
191(10) \\
167(4) \\
245(7) \\
140(7) \\
170(9) \\
160(9) \\
244(7) \\
232(6) \\
242(8) \\
224(6) \\
236(7) \\
299(10) \\
236(7) \\
169(4) \\
228(13) \\
167(8) \\
452(19) \\
137(6) \\
320(18) \\
162(8) \\
227(12) \\
335(23) \\
262(15) \\
306(21) \\
304(17) \\
193(10) \\
238(7) \\
234(7) \\
267(8) \\
245(7) \\
320(11) \\
205(11) \\
311(11) \\
381(14) \\
253(14) \\
367(13) \\
291(18) \\
360(25) \\
378(24) \\
402(31) \\
304(19) \\
181(22) \\
383(28) \\
288(17) \\
196(10) \\
3 \\
\end{array}$ \\
\hline
\end{tabular}


Table Bond longths $[\mathrm{A}]$ and angl a $\left[{ }^{\circ}\right]$ for 1 .

\begin{tabular}{|c|c|c|c|}
\hline 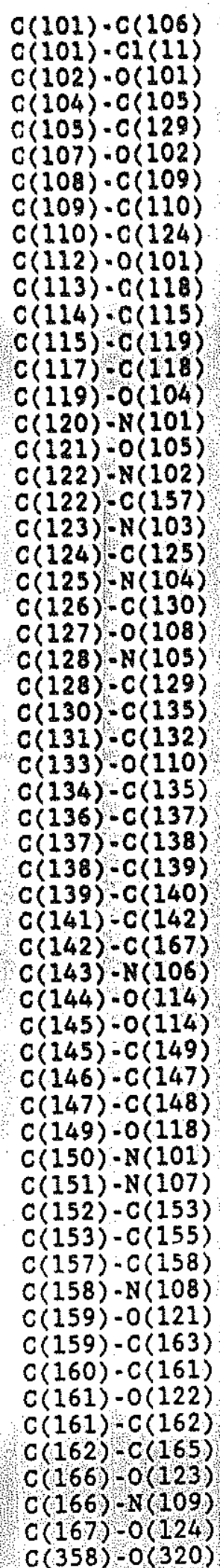 & $\begin{array}{l}1.390(9) \\
1.726(6) \\
1.404(7) \\
1.409(8) \\
1.520(9) \\
1.393(7) \\
1.384(9) \\
1.380(8) \\
1.529(8) \\
1.408(7) \\
1.395(9) \\
1.412(9) \\
1.521(10) \\
1.380(9) \\
1.426(9) \\
1.453(9) \\
1.231(9) \\
1.470(10) \\
1.58(2) \\
1.321(8) \\
1.556(8) \\
1.344(8) \\
1.517(9) \\
1.251(7) \\
1.445(8) \\
1.561(9) \\
1.390(8) \\
1.360(10) \\
1.371(8) \\
1.404(9) \\
1.396(9) \\
1.382(10) \\
1.363(10) \\
1.405(10) \\
1.517(9) \\
1.559(10) \\
1.336(8) \\
1.418(7) \\
1.426(8) \\
1.517(11) \\
1.526(10) \\
1.506(10) \\
1.369(10) \\
1.347(9) \\
1.496(11) \\
1.457(14) \\
1.53(2) \\
1.46(2) \\
1.24(2) \\
1.415(8) \\
1.513(10) \\
1.534(10) \\
1.426(9) \\
1.568(10) \\
1.525(12) \\
1.257(9) \\
1.340(11) \\
1.258(9) \\
1.33(2) \\
\end{array}$ & 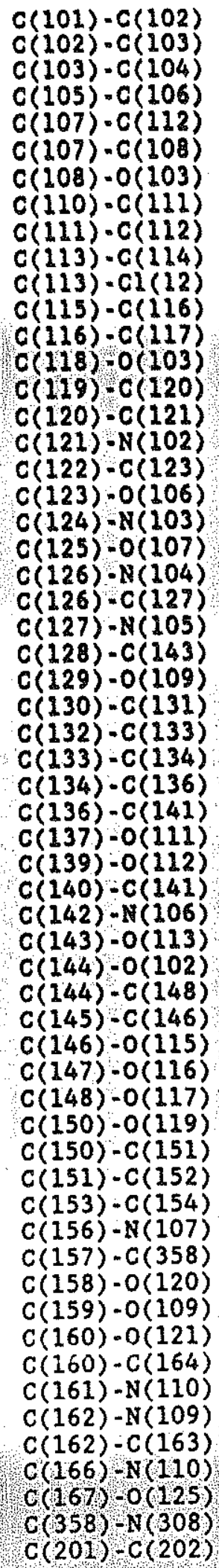 & $\begin{array}{l}1.405(8) \\
1.363(9) \\
1.37(8) \\
1.383(9) \\
1.384(8) \\
1.408(8) \\
1.397(7) \\
1.405(8) \\
1.377(9) \\
1.383(9) \\
1.741(7) \\
1.386(10) \\
1.384(10) \\
1.379(7) \\
1.355(1) \\
1.523(10) \\
1.339(9) \\
1.555(10) \\
1.213(8) \\
1.465(7) \\
1.228(7) \\
1.469(7) \\
1.526(9) \\
1.349(8) \\
1.538(9) \\
1.416(7) \\
1.403(10) \\
1.386(10) \\
1.404(10) \\
1.503(9) \\
1.420(10) \\
1.380(9) \\
1.371(8) \\
1.409(9) \\
1.479(9) \\
1.209(8) \\
1.416(7) \\
1.508(9) \\
1.515(11) \\
1.420(9) \\
1.446(11) \\
1.431(8) \\
1.232(9) \\
1.526(11) \\
1.536(13) \\
1.50(2) \\
1.493(12) \\
1.47(2) \\
1.32(2) \\
1.421(8) \\
1.420(9) \\
1.540(10) \\
1.449(10) \\
1.473(9) \\
1.549(10) \\
1.333(10) \\
1.226(9) \\
1.32(3) \\
1.384(9) \\
\end{array}$ \\
\hline
\end{tabular}






$\mathrm{C}(106) \cdot \mathrm{C}(101) \cdot \mathrm{C}(102)$ $c(102)-c(101)-c 1(1)$ $\mathrm{C}(103)-\mathrm{C}(102)-0(101)$ $C(102)-C(103)-C(104)$
$119.3(6), C(106)-C(101)-C 1(11)$ $120.8(5) \quad \mathrm{C}(103)-\mathrm{C}(102)-\mathrm{C}(101)$ $118.9(5) \quad C(101)-c(102)-0(101)$ $121,0(6) \mathrm{C}(103)-\mathrm{C}(104)-\mathrm{C}(105)$
$1.413(8)$
$1.507(9)$
. $395(9)$
(9)
$1.542(9)$
$1.386(20)$
$1.327(10)$
$1.389(9)$
$1,587(14)$
$1,333(8)$
1.506(9)
$1.247(7)$
$.542(9)$
$1388(9)$
$1.379(9)$
$1.412(9)$
$363(10)$
$.373(11)$
$1.418(11)$
$1.530(11)$
$.333(9)$
$1.457(8)$
$1.541(10)$
$1.512(11)$
$1.384(9)$
$1.346(9)$
$1.497(12)$
$1.46(2)$
$1.22(2)$
1. $493(10)$
1.519 (10)
$1.424(10)$
$1.523(11)$
1.244 (10)
1. 282(11)

$119.9(5)$

$119.8(5)$

$121.3(5)$

120.4 (6) 
$c(106)-c(105)-c(104)$

$c(104) \cdot c(105) \cdot c(129)$

$c(112)-c(107)-0(102)$

$O(102) \cdot c(107) \cdot c(108)$

$c(109) \cdot c(108) \cdot C(107)$

$c(110)-C(109)-C(108)$

$c(109)-C(110)-C(124)$

$c(112)-c(111)-c(110)$

$c(111) \cdot c(112) \cdot 0(101)$

$c(114)-C(113) \cdot C(118)$

$c(118) \cdot c(113)-c 1(12)$

$c(116)-c(115) \cdot c(114)$

$C(114) \cdot C(115) \cdot C(119)$

$c(118) \cdot c(117)-c(116)$

$0(103) \cdot c(118) \cdot c(113)$

$0(104) \cdot C(119) \cdot c(115)$

$c(115)-c(119)-c(120)$

$N(101)-C(120)-C(119)$

$\mathrm{O}(105) \cdot \mathrm{C}(121)-\mathrm{N}(102)$

$N(102)-C(121)-C(120)$

$N(102)-C(122)-C(157)$

$0(106)-C(123)-N(103)$

$\mathrm{N}(103)-\mathrm{C}(123)-\mathrm{C}(122)$

$N(103)-C(124)-C(125)$

$0(107)-c(123)-N(104)$

$N(104) \cdot C(125) \cdot C(124)$

$N(104) \cdot C(126) \cdot C(127)$

$0(108)-C(127)-N(105)$

$N(105) \cdot C(127) \cdot C(126)$

$N(105) \cdot C(128) \cdot C(129)$

$0(109)-c(129)-c(105)$

$C(105) \cdot C(129) \cdot C(128)$

$c(135) \cdot C(130) \cdot c(126)$

$c(132)-c(131)-c(130)$

$o(110)-c(133)-c(132)$

$c(132)-c(133) \cdot c(134)$

$C(133)-C(134)-C(136)$

$c(130) \cdot C(135) \cdot c(134)$

$c(137)-c(136)-c(134)$

$c(138) \cdot c(137)-0(111)$

$O(111)-c(137)-c(136)$

$c(138)-C(139)-0(112)$

$o(112)-C(139)-c(140)$

$C(140)-C(141)-C(136)$

$C(136)-C(141)-C(142)$

$N(106)-C(142)-C(167)$

$O(113)-C(143)-N(106)$

$N(106)-C(143)-C(128)$

$O(102)-C(144)-C(148)$

$O(114) \cdot C(145)-C(146)$

$C(146)-C(145)-C(149)$

$O(115)-C(146)-C(147)$

$o(116)-C(147)-C(148)$

$C(148)-C(147)-C(146)$

$O(117) \cdot C(148)-C(144)$

$O(118)-C(149)-C(145)$

$O(119)-C(150)-C(151)$

$N(107)-C(151)-C(150)$

$\mathrm{C}(150)-\mathrm{C}(151)-\mathrm{C}(152)$

$\mathrm{C}(152), \mathrm{C}(153), \mathrm{C}(154)$

$\mathrm{C}(154)-\mathrm{C}(153)-\mathrm{C}(155)$

$\mathrm{C}(358)-\mathrm{C}(157)-\mathrm{C}(122)$

$\mathrm{N}(108)-\mathrm{C}(158) \mathrm{C}(157)$
$118,3(6)$

$122.3(3)$

$122.9(5)$

$119.4(5)$

$121.0(5)$

$120.3(6)$

$121.5(5)$

$119.7(6)$

$122.0(3)$

$121.3(6)$

$118.5(5)$

$119.4(6)$

$118.8(6)$

$121,0(6)$

$121.2(6)$

$110.0(7)$

$1114(6)$

$111,4(6)$

$122.5(7)$

$117.9(6)$

$112,9(8)$

$122,7(6)$

$118,4(6)$

$110.9(5)$

$123.0(6)$

$114.9(5)$

$106.4(4)$

$119.9(6)$

$121.1(3)$

$111.2(3)$

$113.5(5)$

106.9(5)

$121.7(6)$

$121.0(6)$

$121.6(7)$

$120.4(6)$

$121.4(6)$

$123.7(6)$

$119.6(6)$

$121.6(6)$

$116.7(6)$

$118.0(7)$

121. $2(7)$

$119.8(6)$

$120.7(5)$

$111.7(6)$

$122.4(6)$

$114.8(6)$

$109.9(5)$

$108.3(6)$

$114.6(7)$

$110.0(7)$

$111.3(7)$

$110.2(6)$

$112,3(6)$

$110.5(7)$

$120.0(6)$

$106.2(7)$

$111,0(7)$

113,711

$109.8(14)$

$114,1(1$ $c(106) \cdot C(105) \cdot C(129)$

$C(105)-C(106)-C(101)$

$c(112) \cdot C(107) \cdot C(108)$

$c(109) \cdot C(108) \cdot 0(103)$

$0(103)-C(108)-C(107)$

$c(109) \cdot c(110) \cdot c(111)$

$c(111)-c(110)-c(124)$

$c(111)-c(112)-c(107)$

$c(107) \cdot c(112) \cdot 0(101)$

$c(114)-c(113)-C 1(12)$

$c(113)-c(114)-c(115)$

$c(116)-C(115)-C(119)$

$c(117)-c(116)-c(115)$

$0(103) \cdot c(118) \cdot c(117)$

$c(117)-c(118)-c(113)$

$0(104) \cdot c(119) \cdot c(120)$

$\mathrm{N}(101) \cdot \mathrm{C}(130) \cdot \mathrm{C}(121)$

$c(121) \cdot C(120)-C(119)$

$0(105)-C(121)-C(120)$

$N(102)-C(122)-C(123)$

$c(123)-c(122)-c(157)$

$o(106)-c(123)-c(122)$

$N(103)-C(124)-C(110)$

$c(110)-c(124)-c(125)$

$o(107)-c(125)-c(124)$

$N(104)-C(126)-C(130)$

$c(130)-c(126)-C(127)$

$o(108) \cdot c(127) \cdot c(126)$

$N(105)-C(128)-C(143)$

$C(143) \cdot C(128) \cdot C(129)$

$O(109)-C(129)-C(128)$

$c(135)-c(130)-c(131)$

$C(131)-C(130)-C(126)$

$c(131) \cdot c(132) \cdot c(133)$

$o(110) \cdot C(133) \cdot C(134)$

$c(133)-c(134)-c(135)$

$C(135)-C(134)-C(136)$

$c(137)-c(136)-c(141)$

$c(141)-C(136)-C(134)$

$c(138)-C(137)-C(136)$

$c(139)-c(138)-C(137)$

$c(138)-c(139)-C(140)$

$C(139)-C(140)-C(141)$

$c(140)-c(141)-c(142)$

$N(106)-C(142)-C(141)$

$c(141)-C(142)-C(167)$

$O(113)-C(143)-C(128)$

$O(102) \cdot C(144)-0(114)$

$0(114)-C(144)-C(148)$

$0(114)-C(145)-C(149)$

$O(115)-C(146)-C(145)$

$C(145)-C(146)-C(147)$

$O(116)-C(147)-C(146)$

$0(117)-C(148)-C(147)$

$C(147)-C(148)-C(144)$

$O(110)-C(150)-N(101)$

$N(101)-C(150)-C(151)$

$N(107)-C(151)-C(152)$

$c(153)-C(152)-C(151)$

$C(152)-c(153)-C(155)$

$c(158)-c(157)-c(122)$

$\mathrm{N}(108), \mathrm{C}(158), 0(120)$

$0(120)-c(158) \cdot c(157)$
$110,(3)$

$121.1(6)$

$117,7 \times 5$

$123.5(\$)$

$115: 5(5)$

$119,4(6)$

$119.1(5)$

$121.9(5)$

$116,1(5)$

$120.2(5)$

$119,0(6)$

$121,5(6)$

$120,3(6)$

$120.2(6)$

$118.6(6)$

$107,0(6)$

$113.4(6)$

$110.9(6)$

$119.6(7)$

$113,7(7)$

$109,4(8)$

$118.7(7)$

$112,4(5)$

$108,3(5)$

$121.9(6)$

$116.8(5)$

$107.9(5)$

$118,9(5)$

$109.5(5)$

$114.6(5)$

$108.2(5)$

$116.9(6)$

$120.9(5)$

$121.4(7)$

$118.0(6)$

$116.5(6)$

$122.0(6)$

$118.2(5)$

$122.3(6)$

$121.7(7)$

$120.2(7)$

$120.7(6)$

$119.3(6)$

$119.4(6)$

$110,6(6)$

$114.2(5)$

$122.5(6)$

$106.9(5)$

$110.9(5)$

$107.9(6)$

$110.7(7)$

$109.7(6)$

$107.7(6)$

$108.2(6)$

$110.0(6)$

$124.4(7)$

$115.7(7)$

113.3(7)

$118.2(8)$

$1128(9)$

$113,5(11)$

122 ( 2 )

$119(2)$ 


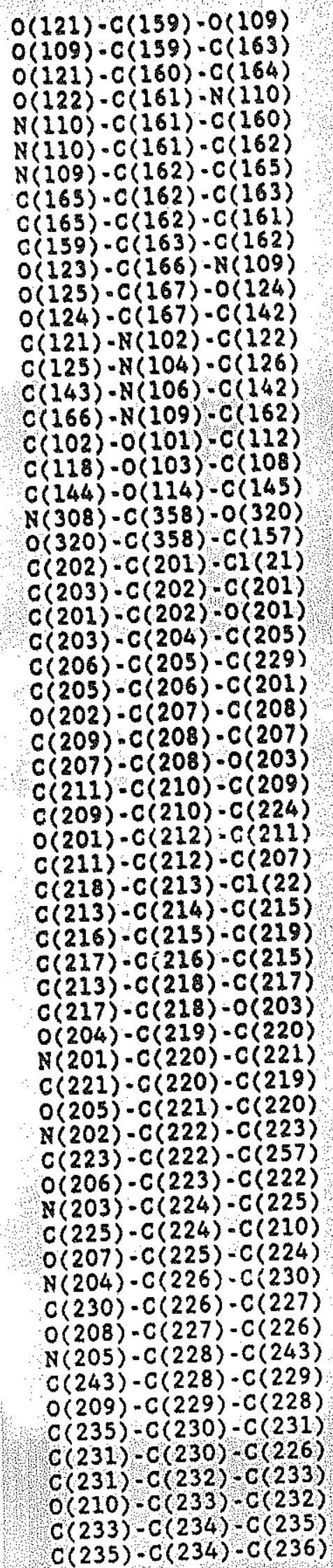

$112.0(3)$

$110.0(6)$

$106.7(7)$

$109.4(6)$

$115.9(7)$

$99.2(5)$

$112.2(7)$

$111.3(6)$

$114.5(7)$

$114.5(6)$

$126.0(8)$

$125.1(8)$

$117.3(7)$

$122,3(7)$

$122.4(5)$

$120,5(6)$

$111,3(6)$

$116.1(4)$

$115.6(5)$

111:3(5)

$104(3)$

$130(2)$

$120,2(5)$

$120,1(6)$

$120.3(6)$

$121.7(6)$

$119.8(5)$

$120.6(6)$

$119.9(5)$

$122.3(6)$

$116.0(5)$

$119.7(6)$

$119.5(5)$

$123.2(5)$

$120.1(6)$

$119.9(5)$

$120.0(7)$

$120.8(7)$

$120.2(7)$

$120.4(6)$

$118.9(6)$

$106.6(6)$

$113.0(6)$

$111.1(6)$

$121.2(7)$

$113.4(6)$

$108.0(7)$

$119.5(6)$

$109.6(5)$

$107.6(5)$

$120.6(6)$

$117.2(5)$

$107.4(5)$

$119.9(5)$

109.8(6)

$115.0(5)$

$106.4(5)$

$117.2(6)$

$121.0(6)$

$120.7(8)$

$121.8(8)$

$117.3(6)$

$119.4(7)$
$0(121) \cdot C(159)-C(163)$

$0(121)-C(160) \cdot C(161)$

$C(161)-C(160) \cdot C(164)$

$O(122) \cdot C(161) \cdot C(160)$

$o(122)-c(161)-C(162)$

$c(160)-C(161)-C(162)$

$N(109)-C(162)-C(163)$

$N(109) \cdot C(162) \cdot C(161)$

$c(163)-c(162)-c(161)$

$O(123)-C(166)-N(110)$

$N(110) \cdot C(166) \cdot N(109)$

$O(125)-C(167)-C(142)$

$C(150)-N(101)-C(120)$

$C(123)-N(103)-C(124)$

$C(127)-N(105)-C(128)$

$C(156) \cdot N(107) \cdot C(151)$

$C(166) \cdot N(110)-C(161)$

$C(107)-0(102)-C(144)$

$C(129)-0(109)-C(159)$

$C(159)-0(121)-C(160)$

$N(308)-C(358)-C(157)$

$C(202)-C(201) \cdot C(206)$

$C(206)-C(201)-C I(21)$

$c(203) \cdot c(202)-0(201)$

$c(202)-c(203)-c(204)$

$c(206)-c(205)-c(204)$

$c(204)-c(205)-c(229)$

$O(202)-C(207) \cdot C(212)$

$c(212)-C(207)-C(208)$

$C(209)-C(208) \cdot 0(203)$

$c(208)-c(209)-C(210)$

$c(211)-C(210) \cdot C(224)$

$c(210)-c(211)-c(212)$

$0(201)-C(212)-C(207)$

$C(218)-C(213)-C(214)$

$C(214) \cdot C(213) \cdot C L(22)$

$C(216)-C(215) \cdot C(214)$

$c(214)-C(215)-C(219)$

$C(216)-C(217)-C(218)$

$c(213)-c(218)-0(203)$

$o(204)-c(219)-c(215)$

$c(215)-C(219)-C(220)$

$N(201)-C(220)-C(219)$

$O(205)-C(221)-N(202)$

$N(202)-C(221)-C(220)$

$N(202)-C(222)-C(257)$

$O(206)-C(223)-N(203)$

$N(203)-C(223)-C(222)$

$N(203)-C(224)-C(210)$

$\mathrm{O}(207)-\mathrm{C}(225)-\mathrm{N}(204)$

$N(204)-C(225)-C(224)$

$\mathrm{N}(204)-\mathrm{C}(226)-\mathrm{C}(227)$

$O(208)-C(227)-N(205)$

$N(205)-C(227)-C(226)$

$N(205)-C(228)-C(229)$

$\mathrm{O}(209) \cdot C(\angle 29) \cdot C(205)$

$\mathrm{C}(205)-\mathrm{C}(229)-\mathrm{C}(228)$

$c(235)-c(230)-c(226)$

$c(232)-c(231)-c(230)$

$0(210)-c(233)-c(234)$

$c(234)-C(233)-c(232)$

$c(233)-C(234)-C(236)$

$C(230)-C(235)-C(234)$
$111,0(5)$

$112,8(6)$

$111,0(6)$

$105,4(6)$

$112.2(6)$

$114.8(5)$

$108.1(6)$

$99.9(6)$

$110.1(6)$

$125,9(8)$

$108.1(7)$

$117.5(7)$

$120.5(6)$

$122.1(5)$

$126.4(5)$

$114.6(7)$

$112,6(7)$

$114.8(4)$

$113.5(5)$

$115.1(6)$

$103(3)$

$119.8(6)$

$120.0(5)$

$119.6(5)$

$120,6(6)$

$117.0(6)$

$122.9(6)$

$121.8(5)$

$118.2(6)$

$121,7(6)$

$118.4(6)$

$120.8(5)$

$121.1(6)$

$116.6(5)$

$120.4(6)$

$119.6(5)$

$119.1(7)$

$119.9(7)$

$119.5(7)$

$120.7(6)$

$113.3(7)$

$112.4(6)$

$111.1(6)$

$122.2(6)$

$116.5(6)$

$111.1(7)$

$122.5(6)$

$117.8(6)$

$112.5(5)$

$122.9(6)$

$116.4(5)$

$107.3(5)$

$120.0(6)$

$120.0(5)$

$110.5(5)$

$113.2(5)$

108.2(5)

$120.8(6)$

$120.4(7)$

$116.6(7)$

$121.5(7)$

$123.2(6)$

$122,7(7)$ 


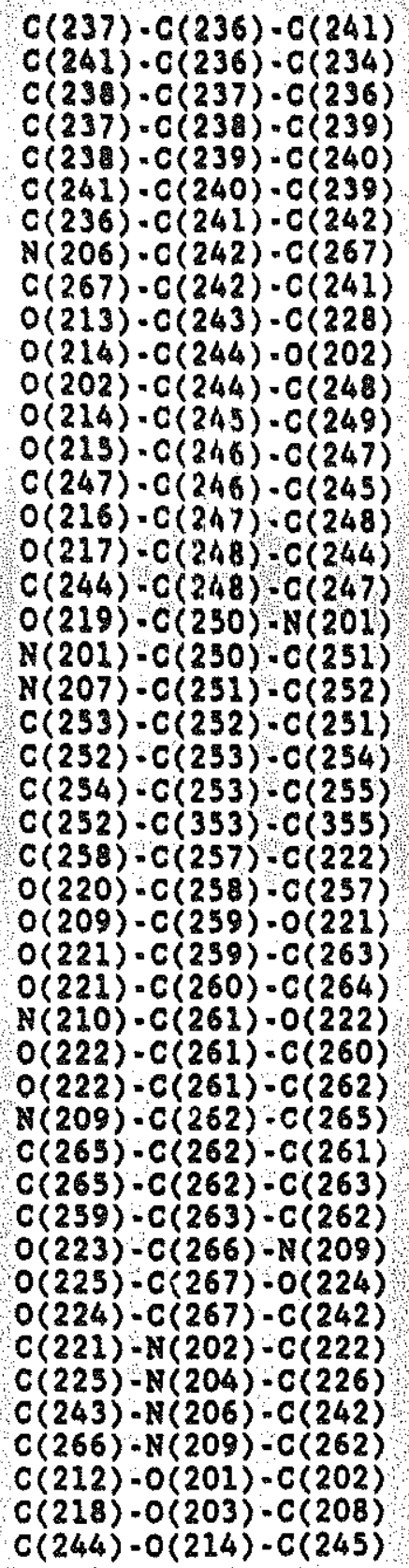

$118.6(6)$

$121,1(7)$

$120.9(7)$

$120.9(8)$

$120.7(7)$

$117.6(7)$

$120.1(6)$

$113.7(7)$

$112.9(6)$

$122.0(6)$

$106.4(5)$

$106.4(5)$

$108.8(6)$

$110.1(6)$

$110.1(6)$

$110,8(7)$

$110,3(6)$

$108.6(6)$

$123,8(8)$

$115,3(7)$

$108 \cdot 5(10)$

$135(2)$

$121(2)$

108 (2)

$112(3)$

$113.1(8)$

$123,9(13)$

$111.0(5)$

$111.4(5)$

$106.1(6)$

$106.7(6)$

$107,7(6)$

$111,1(6)$

$112.3(7)$

$114.3(7)$

$112.5(6)$

$115.4(6)$

$126.5(8)$

$124.4(9)$

$117.1(9)$

$122.2(6)$

$121.9(5)$

$120.6(7)$

111.0(6)

$115.5(5)$

$114.7(5)$

$112.0(5)$
$C(237)-C(236)-C(234)$

$c(238) \cdot c(237) \cdot 0(211)$

$O(211)-C(237)-C(236)$

$c(238)-C(239)=0(212)$

$O(212)-C(239)-C(240)$

$C(236)-C(241)-C(240)$

$C(240)-C(241) \cdot C(242)$

$N(206)-C(242) \cdot C(241)$

$O(213)-C(243)-N(206)$

$N(206)=C(243)=C(228)$

$O(214) \cdot C(244) \cdot C(248)$

$O(214) \cdot C(245) \cdot C(246)$

$C(246) \cdot C(245) \cdot C(249)$

$O(215)-C(246)-C(245)$

$O(216) \cdot C(247) \cdot c(246)$

$C(246)-C(247)-C(248)$

$0(217)-C(248)-C(247)$

$0(218) \cdot c(249) \cdot c(245)$

$o(219)-c(250)-c(251)$

$N(207) \cdot C(251) \cdot C(250)$

$c(250)-c(251)-c(252)$

$c(353)-c(252)-c(251)$

$c(252)-c(253)-c(255)$

$c(252)-c(353) \cdot c(354)$

$C(354)-C(353)-C(355)$

$O(220)-C(258)-N(208)$

$N(208)-C(258)-C(257)$

$O(209)=c(259)-C(263)$

$O(221)-C(260) \cdot C(261)$

$C(261) \cdot C(260) \cdot C(264)$

$N(210) \cdot C(261) \cdot C(260)$

$N(210)-C(261)-C(262)$

$C(260)-C(261)-C(262)$

$N(209)-C(262)-C(261)$

$N(209)-C(262)-C(263)$

$c(261)-C(262)-C(263)$

$O(223)-c(266)-N(210)$

$N(210)-C(266)-H(209)$

$0(225) \cdot c(267) \cdot c(242)$

$C(250)-N(201)-C(220)$

$C(223) \cdot N(203) \cdot C(224)$

$C(227)-N(205)-C(228)$

$C(256)-N(207)-C(251)$

$C(266)-N(210)-C(261)$

$C(207)-0(202)-C(244)$

$C(259) \cdot 0(209) \cdot C(229)$

$C(260) \cdot 0(221) \cdot C(259)$
$120,2(6)$

$118.8(7)$

$120,3(6)$

$121.6(7)$

$117.6(7)$

$120.8(7)$

$119.1(7)$

$109.4(6)$

$12 \div, 4(7)$

$114.4(7)$

$110.1(5)$

$107.3(6)$

$116,7(6)$

$113.0(6)$

$109.8(7)$

$111,8(6)$

$109,3(6)$

$112,8(7)$

$120.9(7)$

$107,8(8)$

$109,6(9)$

$124(2)$

$115(2)$

$122(3)$

$108(3)$

$124(2)$

112(2)

$109.7(6)$

$113.4(6)$

$112.1(6)$

$115.7(6)$

$101.1(6)$

$114.3(6)$

$99.1(6)$

$108,9(6)$

$108,9(6)$

$126,6(8)$

$106.9(7)$

$118.4(9)$

$121.0(6)$

$122.1(5)$

$125.9(6)$

$113.9(9)$

111.5(6)

$113.0(4)$

$115.3(5)$

$114.2(5)$

Symatry transformations used to generate equivalent atons: 
Tabl- Anlsotrople diaplecement parameters $\left(\lambda^{2}\right.$ a $\left.10^{3}\right)$ for 1 . The anisotrople dieplocoment faotor exponent takes the form:

( $\left(\mathrm{ha}^{*}\right)^{2} v_{11}+\ldots+2 h k{ }^{*} b^{*} v_{12}$ )

\begin{tabular}{|c|c|c|c|c|c|c|}
\hline & U11 & U22 & U33 & U23 & U13 & U12 \\
\hline $\begin{array}{l}c(101) \\
c(102) \\
c(103) \\
c(104) \\
c(105) \\
c(106) \\
c(107) \\
c(108) \\
c(109) \\
c(110) \\
c(111) \\
c(112) \\
c(113) \\
c(114) \\
c(115) \\
c(116) \\
c(117) \\
c(118) \\
c(119) \\
c(120) \\
c(121) \\
c(122) \\
c(123) \\
c(124) \\
c(125) \\
c(126) \\
c(127) \\
c(128) \\
c(129) \\
c(130) \\
c(131) \\
c(132) \\
c(133) \\
c(134) \\
c(135) \\
c(136) \\
c(137) \\
c(138) \\
c(139) \\
c(140) \\
c(141) \\
c(142) \\
c(143) \\
c(144) \\
c(145) \\
c(146) \\
c(147) \\
c(148) \\
c(149) \\
c(150) \\
c(151)\end{array}$ & $\begin{array}{l}40(4) \\
52(4) \\
30(4) \\
45(4) \\
47(4) \\
41(3) \\
38(3) \\
42(4) \\
42(4) \\
33(3) \\
36(3) \\
44(4) \\
43(4) \\
64(5) \\
59(4) \\
43(4) \\
43(4) \\
46(4) \\
76(5) \\
66(4) \\
63(4) \\
56(4) \\
56(4) \\
30(3) \\
42(4) \\
44(4) \\
47(4) \\
49(4) \\
49(4) \\
47(4) \\
58(5) \\
51(5) \\
61(5) \\
51(4) \\
34(3) \\
50(4) \\
70(5) \\
78(6) \\
54(4) \\
62(5) \\
52(4) \\
58(4) \\
57(4) \\
34(3) \\
53(4) \\
44(4) \\
45(4) \\
45(4) \\
59(5) \\
60(5) \\
666(5) \\
72(6)\end{array}$ & $\begin{array}{l}31(4) \\
30(4) \\
45(4) \\
43(4) \\
33(4) \\
39(4) \\
34(3) \\
41(4) \\
32(4) \\
34(3) \\
35(3) \\
30(3) \\
49(4) \\
52(4) \\
64(4) \\
53(4) \\
47(4) \\
37(4) \\
65(5) \\
45(4) \\
52(5) \\
45(5) \\
48(4) \\
33(3) \\
35(4) \\
35(4) \\
40(4) \\
49(4) \\
40(4) \\
35(4) \\
63(5) \\
58(5) \\
59(5) \\
47(4) \\
43(4) \\
62(4) \\
63(5) \\
73(5) \\
65(5) \\
61(5) \\
57(4) \\
65(5) \\
53(4) \\
35(4) \\
72(5) \\
58(5) \\
67(6) \\
49(5) \\
73(5) \\
59(5) \\
56(5) \\
88(7) \\
\end{array}$ & $\begin{array}{l}48(3) \\
47(3) \\
45(4) \\
42(3) \\
36(3) \\
43(3) \\
32(3) \\
43(3) \\
50(4) \\
37(3) \\
45(3) \\
46(4) \\
60(4) \\
52(4) \\
55(4) \\
66(4) \\
54(4) \\
59(4) \\
64(5) \\
56(4) \\
61(4) \\
138(7) \\
52(4) \\
36(3) \\
37(3) \\
32(3) \\
38(3) \\
32(3) \\
28(3) \\
33(3) \\
39(4) \\
55(4) \\
54(4) \\
39(3) \\
39(3) \\
38(3) \\
55(4) \\
51(4) \\
36(4) \\
38(3) \\
39(3) \\
39(4) \\
38(3) \\
49(4) \\
48(4) \\
78(5) \\
76(5) \\
64(4) \\
55(4) \\
69(5) \\
77(5) \\
66(5) \\
\end{array}$ & $\begin{array}{c}8(3) \\
13(3) \\
21(3) \\
16(3) \\
9(3) \\
9(3) \\
7(3) \\
14(3) \\
10(3) \\
8(3) \\
10(3) \\
10(3) \\
20(3) \\
15(3) \\
27(3) \\
19(4) \\
9(3) \\
14(3) \\
22(4) \\
11(3) \\
16(4) \\
-6(5) \\
7(3) \\
8(3) \\
10(3) \\
6(3) \\
11(3) \\
18(3) \\
10(3) \\
7(3) \\
14(3) \\
4(4) \\
25(4) \\
17(3) \\
16(3) \\
23(3) \\
34(4) \\
33(4) \\
22(3) \\
15(3) \\
24(3) \\
24(3) \\
17(3) \\
22(3) \\
2(4) \\
-7(4) \\
-15(4) \\
4(3) \\
-11(4) \\
24(4) \\
25(4) \\
24(5)\end{array}$ & $\begin{array}{l}21(3) \\
37(3) \\
32(3) \\
24(3) \\
22(3) \\
23(3) \\
19(3) \\
28(3) \\
25(3) \\
17(3) \\
24(3) \\
26(3) \\
30(3) \\
34(4) \\
33(4) \\
24(4) \\
26(3) \\
38(3) \\
33(4) \\
22(4) \\
23(4) \\
15(5) \\
19(4) \\
19(3) \\
22(3) \\
21(3) \\
27(3) \\
23(3) \\
20(3) \\
20(3) \\
25(4) \\
20(4) \\
35(4) \\
22(3) \\
22(3) \\
23(3) \\
38(4) \\
34(4) \\
17(3) \\
30(3) \\
24(3) \\
29(3) \\
25(3) \\
19(3) \\
13(4) \\
26(4) \\
28(4) \\
33(3) \\
19(4) \\
15(4) \\
19(4) \\
13(5) \\
\end{array}$ & $\begin{array}{l}18(3) \\
20(3) \\
26(3) \\
30(3) \\
23(3) \\
16(3) \\
13(3) \\
22(3) \\
20(3) \\
18(3) \\
17(3) \\
21(3) \\
25(3) \\
33(4) \\
45(4) \\
25(4) \\
13(4) \\
23(3) \\
47(4) \\
34(4) \\
33(4) \\
31(4) \\
34(4) \\
15(3) \\
17(3) \\
16(3) \\
24(3) \\
34(3) \\
23(3) \\
16(3) \\
15(4) \\
87(4) \\
24(4) \\
22(3) \\
16(3) \\
22(4) \\
40(4) \\
45(5) \\
21(4) \\
28(4) \\
25(4) \\
31(4) \\
33(4) \\
17(3) \\
31(4) \\
18(4) \\
14(4) \\
20(4) \\
19(5) \\
28(4) \\
31(4) \\
18(5)\end{array}$ \\
\hline
\end{tabular}




\begin{tabular}{|c|c|c|c|c|c|c|}
\hline 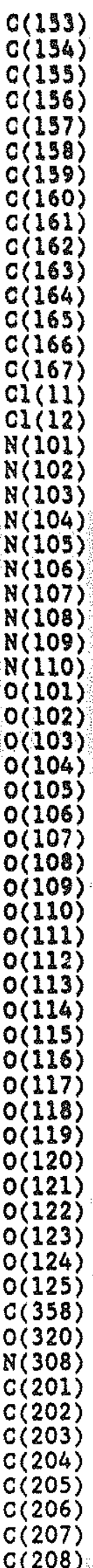 & $\begin{array}{c}94(8) \\
102(9) \\
301(24) \\
85(7) \\
77(7) \\
120(11) \\
73(3) \\
69(4) \\
73(5) \\
81(5) \\
93(5) \\
117(8) \\
70(5) \\
127(8) \\
76(5) \\
57(1) \\
72(1) \\
58(4) \\
54(3) \\
39(3) \\
37(3) \\
51(3) \\
48(3) \\
67(4) \\
129(13) \\
104(5) \\
98(5) \\
55(3) \\
33(2) \\
52(3) \\
81(4) \\
66(4) \\
85(4) \\
39(3) \\
88(4) \\
55(3) \\
91(4) \\
111(5) \\
93(4) \\
39(3) \\
40(3) \\
47(3) \\
58(4) \\
50(3) \\
113(3) \\
106(5) \\
126(11) \\
63(3) \\
95(4) \\
219(9) \\
88(4) \\
113(5) \\
192(21) \\
196(17) \\
205(25) \\
64(5) \\
42(4) \\
46(4) \\
39(4) \\
45(4) \\
57(4) \\
39(3) \\
42(4)\end{array}$ & $\begin{array}{c}160(9) \\
248(17) \\
136(10) \\
157(12) \\
62(7) \\
170(16) \\
41(4) \\
61(5) \\
72(5) \\
84(3) \\
64(5) \\
121(8) \\
115(7) \\
100(6) \\
74(5) \\
102(2) \\
63(1) \\
53(4) \\
46(4) \\
27(3) \\
36(3) \\
46(3) \\
58(4) \\
80(5) \\
196(18) \\
109(5) \\
75(4) \\
31(2) \\
33(2) \\
39(3) \\
87(4) \\
128(6) \\
79(4) \\
79(4) \\
79(4) \\
45(3) \\
73(4) \\
83(4) \\
108(5) \\
97(4) \\
58(3) \\
127(6) \\
80(5) \\
73(4) \\
90(4) \\
76(4) \\
210(19) \\
49(3) \\
107(5) \\
118(5) \\
118(5) \\
69(4) \\
189(19) \\
194(16) \\
177(19) \\
45(4) \\
47(4) \\
51(4) \\
42(4) \\
50(4) \\
55(4) \\
43(4) \\
35(4)\end{array}$ & $\begin{array}{c}64(5) \\
94(8) \\
87(9) \\
122(8) \\
241(9) \\
202(11) \\
67(4) \\
45(4) \\
48(4) \\
56(4) \\
59(4) \\
45(4) \\
69(5) \\
71(5) \\
44(4) \\
71(1) \\
61(1) \\
63(3) \\
69(4) \\
47(3) \\
34(3) \\
30(3) \\
35(3) \\
76(5) \\
246(18) \\
67(4) \\
62(4) \\
59(3) \\
48(2) \\
77(3) \\
114(5) \\
52(3) \\
91(4) \\
41(3) \\
26(2) \\
52(3) \\
74(4) \\
70(3) \\
37(3) \\
58(3) \\
54(3) \\
82(4) \\
154(6) \\
65(3) \\
68(4) \\
91(4) \\
210(16) \\
62(3) \\
79(4) \\
110(5) \\
83(4) \\
71(4) \\
205(12) \\
136(13) \\
254(27) \\
48(4) \\
39(3) \\
38(3) \\
37(3) \\
32(3) \\
35(3)\end{array}$ & $\begin{array}{c}42(6) \\
53(10) \\
23(9) \\
69(8) \\
83(8) \\
169(11) \\
18(3) \\
14(3) \\
24(3) \\
29(4) \\
33(4) \\
22(5) \\
19(5) \\
42(5) \\
27(4) \\
34(1) \\
18(1) \\
20(3) \\
0(3) \\
8(2) \\
2(2) \\
14(2) \\
16(3) \\
21(4) \\
184(15) \\
38(4) \\
16(3) \\
14(2) \\
13(2) \\
17(2) \\
42(4) \\
11(3) \\
-27(3) \\
15(2) \\
10(2) \\
18(2) \\
30(3) \\
38(3) \\
37(3) \\
42(3) \\
44(2) \\
466(4) \\
39(4) \\
88(3) \\
8(3) \\
25(3) \\
148(14) \\
11(2) \\
51(3) \\
61(4) \\
39(4) \\
15(3) \\
133(17) \\
94(11) \\
86(22) \\
20(3) \\
11(3) \\
8(3) \\
3(3) \\
4(3) \\
12(3) \\
14(3) \\
13(3) \\
\end{array}$ & $\begin{array}{c}23(6) \\
53(8) \\
79(13) \\
64(7) \\
75(8) \\
109(11) \\
47(4) \\
25(4) \\
38(4) \\
41(4) \\
54(4) \\
28(5) \\
33(4) \\
66(5) \\
38(4) \\
34(1) \\
16(1) \\
24(3) \\
12(3) \\
18(3) \\
16(2) \\
21(2) \\
24(3) \\
13(4) \\
152(14) \\
43(4) \\
46(4) \\
40(2) \\
21(2) \\
47(3) \\
54(4) \\
8(3) \\
218(4) \\
15(2) \\
15(2) \\
32(2) \\
54(3) \\
48(3) \\
25(3) \\
33(3) \\
22(2) \\
-2(3) \\
74(4) \\
34(3) \\
37(4) \\
19(4) \\
82(11) \\
39(3) \\
62(3) \\
108(6) \\
60(4) \\
54(4) \\
107(16) \\
102(12) \\
15(23) \\
34(3) \\
26(3) \\
22(3) \\
12(3) \\
17(3) \\
20(3) \\
25(3) \\
23(3) \\
\end{array}$ & $\begin{array}{l}50(8) \\
31(11) \\
106(16) \\
66(8) \\
28(6) \\
105(12) \\
33(4) \\
41(4) \\
51(4) \\
63(5) \\
56(4) \\
86(7) \\
55(5) \\
94(6) \\
41(4) \\
53(1) \\
40(1) \\
35(3) \\
31(3) \\
17(3) \\
17(3) \\
30(3) \\
22(3) \\
38(4) \\
115(14) \\
88(5) \\
60(4) \\
21(2) \\
13(2) \\
24(2) \\
63(4) \\
26(4) \\
59(4) \\
42(3) \\
62(3) \\
32(2) \\
11(3) \\
68(4) \\
53(4) \\
47(3) \\
16(2) \\
25(4) \\
-12(3) \\
16(3) \\
67(4) \\
62(4) \\
85(14) \\
\text { (3) }\end{array}$ \\
\hline
\end{tabular}




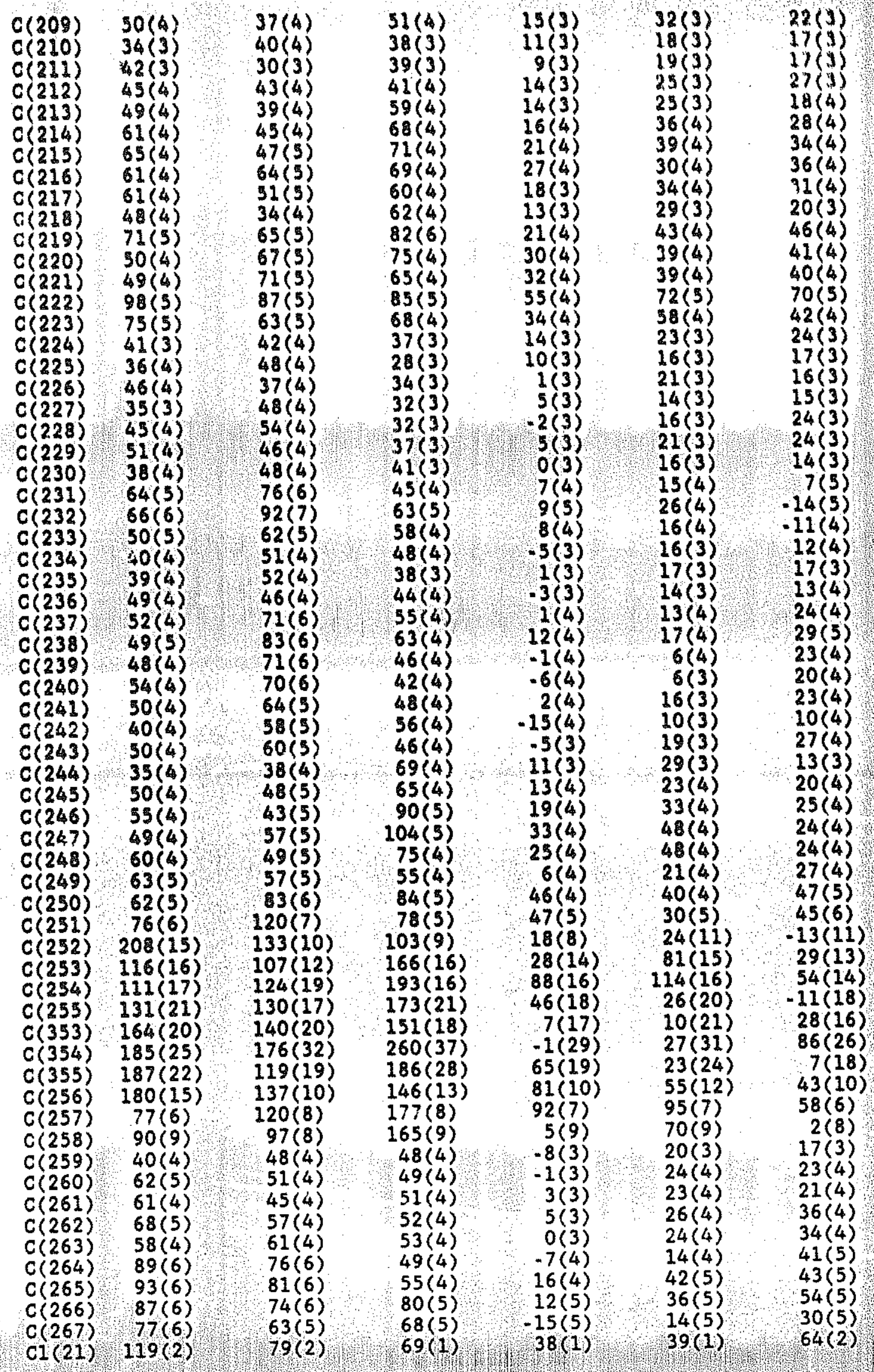




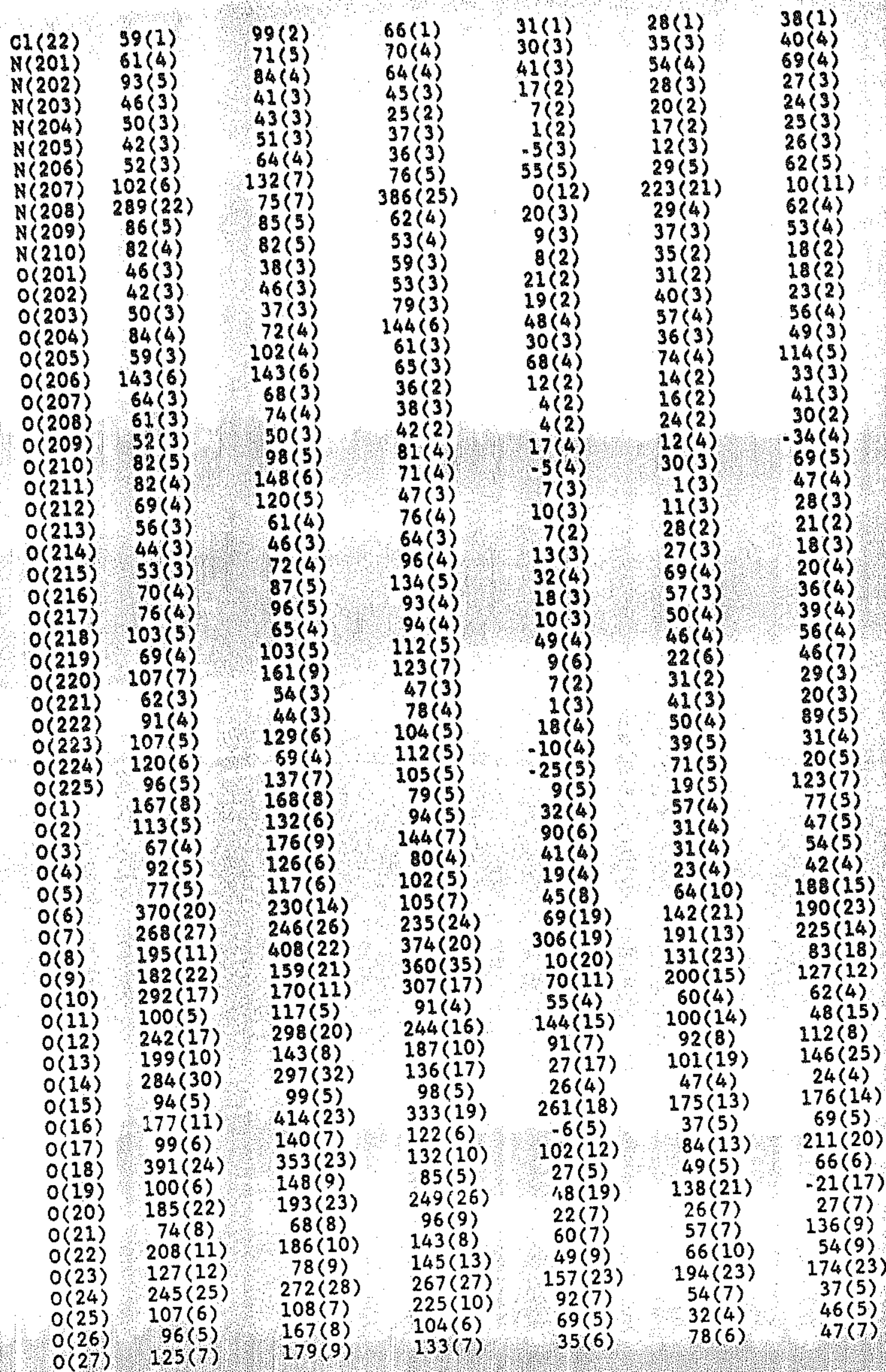




\begin{tabular}{|c|c|c|c|c|c|c|}
\hline 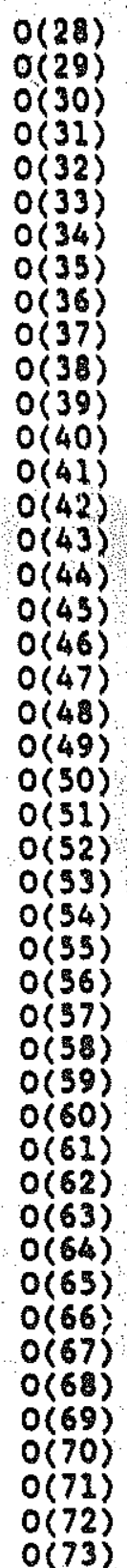 & $\begin{array}{l}260(30) \\
327(29) \\
128(7) \\
323(19) \\
162(18) \\
223(21) \\
203(20) \\
376(21) \\
286(16) \\
169(10) \\
181(11) \\
332(19) \\
250(17) \\
367(18) \\
111(7) \\
182(22) \\
131(15) \\
479(37) \\
93(11) \\
292(31) \\
173(18) \\
288(28) \\
351(40) \\
229(27) \\
262(31) \\
272(28) \\
232(25) \\
216(14) \\
209(13) \\
218(15) \\
194(13) \\
384(25) \\
179(19) \\
281(18) \\
391(28) \\
221(26) \\
414(30) \\
324(35) \\
301(38) \\
323(36) \\
467(32) \\
239(31) \\
148(34) \\
347(41) \\
253(28) \\
127(17)\end{array}$ & $\begin{array}{l}266(31) \\
212(21) \\
156(8) \\
195(13) \\
120(13) \\
99(13) \\
109(14) \\
2199(13) \\
201(13) \\
213(13) \\
288(17) \\
159(11) \\
276(19) \\
227(13) \\
224(11) \\
336(33) \\
171(18) \\
288(23) \\
143(14) \\
287(30) \\
194(19) \\
97(15) \\
306(38) \\
378(39) \\
337(38) \\
271(30) \\
211(23) \\
237(17) \\
263(16) \\
240(17) \\
310(19) \\
314(22) \\
180(20) \\
433(27) \\
372(28) \\
226(25) \\
366(27) \\
389(41) \\
415(48) \\
322(37) \\
338(40) \\
324(40) \\
209(44) \\
383(44) \\
241(29) \\
188(20)\end{array}$ & $\begin{array}{l}298(32) \\
100(12) \\
148(8) \\
289(17) \\
154(16) \\
223(23) \\
178(19) \\
291(16) \\
290(16) \\
181(11) \\
131(8) \\
164(10) \\
306(21) \\
68(5) \\
194(10) \\
135(17) \\
152(16) \\
606(42) \\
191(16) \\
302(30) \\
131(13) \\
309(27) \\
444(47) \\
201(25) \\
260(33) \\
382(31) \\
193(20) \\
227(14) \\
199(12) \\
266(18) \\
187(12) \\
268(18) \\
335(32) \\
202(14) \\
350(27) \\
211(23) \\
398(29) \\
243(30) \\
349(44) \\
455(40) \\
420(49) \\
326(38) \\
162(35) \\
428(49) \\
293(30) \\
216(23)\end{array}$ & $\begin{array}{r}15(23) \\
46(12) \\
88(7) \\
112(12) \\
57(12) \\
47(13) \\
26(12) \\
92(12) \\
123(12) \\
115(10) \\
53(9) \\
32(8) \\
108(16) \\
29(6) \\
142(9) \\
76(19) \\
39(13) \\
166(25) \\
99(13) \\
31(24) \\
58(12) \\
50(17) \\
83(30) \\
89(24) \\
185(30) \\
75(26) \\
99(17) \\
59(12) \\
24(10) \\
50(13) \\
55(12) \\
81(16) \\
79(20) \\
14(15) \\
89(21) \\
43(19) \\
161(22) \\
90(26) \\
116(34) \\
108(31) \\
168(35) \\
106(30) \\
8(28) \\
161(35) \\
35(23) \\
68(17)\end{array}$ & $\begin{array}{c}23(22) \\
92(15) \\
36(6) \\
195(16) \\
79(14) \\
127(19) \\
134(17) \\
252(16) \\
202(14) \\
41(8) \\
48(8) \\
108(12) \\
71(15) \\
27(8) \\
75(7) \\
58(16) \\
61(13) \\
223(29) \\
81(12) \\
142(26) \\
83(12) \\
194(23) \\
250(35) \\
77(20) \\
55(22) \\
203(24) \\
158(19) \\
103(11) \\
125(11) \\
101(13) \\
87(10) \\
217(19) \\
172(21) \\
119(13) \\
112(21) \\
86(20) \\
238(24) \\
129(25) \\
119(30) \\
168(29) \\
141(33) \\
151(28) \\
98(29) \\
109(30) \\
97(23) \\
75(16)\end{array}$ & $\begin{array}{r}215(213) \\
210(23) \\
44(6) \\
145(14) \\
85(13) \\
102(14) \\
57(14) \\
195(15) \\
196(12) \\
2(9) \\
100(11) \\
108(12) \\
150(16) \\
202(14) \\
88(7) \\
131(22) \\
53(14) \\
236(26) \\
60(11) \\
111(26) \\
99(16) \\
75(17) \\
210(32) \\
207(28) \\
162(27) \\
124(24) \\
129(19) \\
125(13) \\
98(12) \\
87(13) \\
111(13) \\
138(19) \\
116(17) \\
199(19) \\
255(25) \\
66(21) \\
224(24) \\
268(33) \\
221(36) \\
175(30) \\
295(40) \\
120(27) \\
699(30) \\
276(38) \\
121(24) \\
50(15)\end{array}$ \\
\hline
\end{tabular}


Toblo 10. Hydrogen coordinates ( $10^{4}$ ) and leotropla displocoment parameter $\left(A^{2} \times 10^{3}\right)$ for 1 .

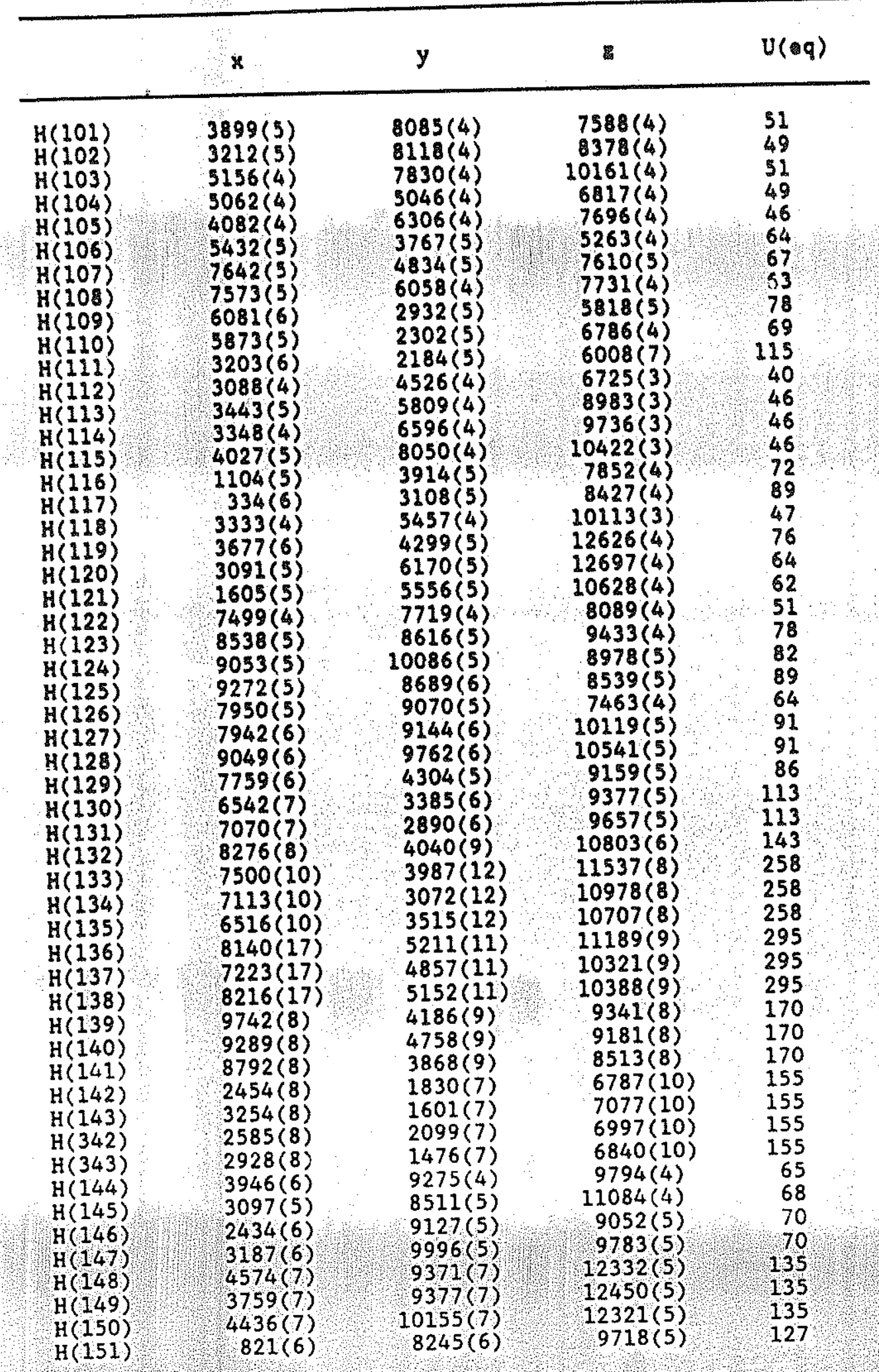




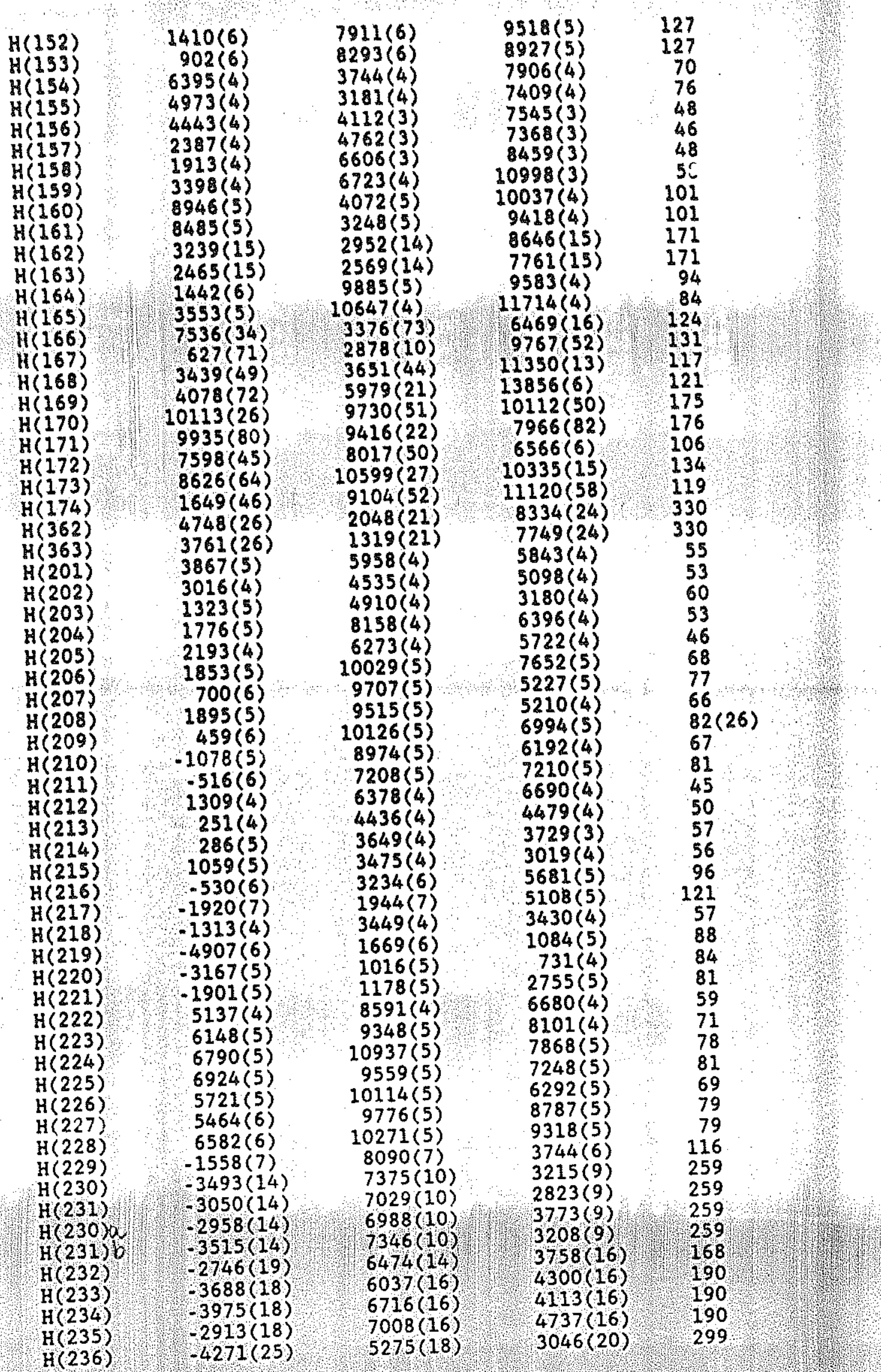




\begin{tabular}{|c|c|c|c|c|}
\hline 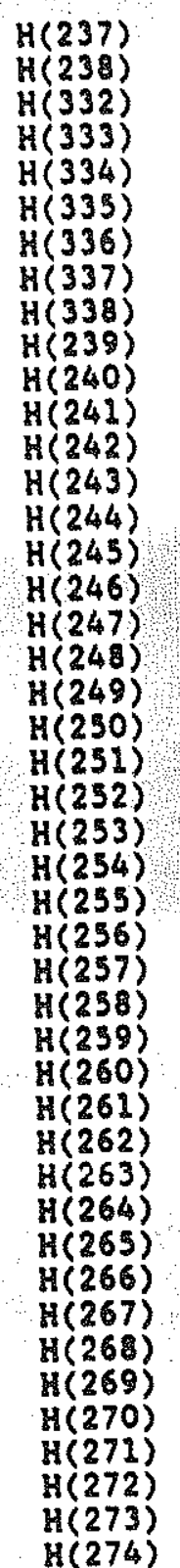 & 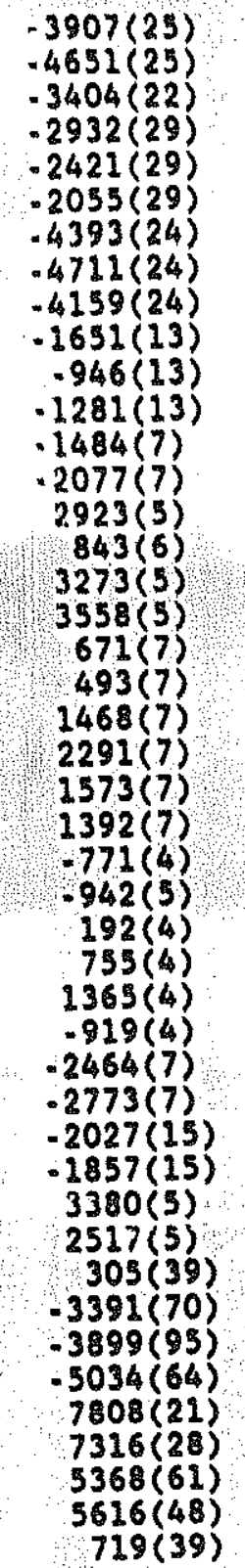 & $\begin{array}{l}5614(18) \\
5790(18) \\
6866(18) \\
5919(28) \\
6134(28) \\
6831(28) \\
5405(19) \\
5961(19) \\
5592(19) \\
9923(10) \\
9384(10) \\
9822(10) \\
5701(7) \\
6137(7) \\
3824(5) \\
1835(5) \\
3022(3) \\
2990(5) \\
2054(6) \\
1136(6) \\
1857(6) \\
1721(6) \\
874(6) \\
1634(6) \\
8251(4) \\
7406(4) \\
6988(3) \\
5011(3) \\
3277(4) \\
2614(4) \\
8502(6) \\
8722(6) \\
4654(8) \\
4709(8) \\
1406(5) \\
1564(4) \\
10767(10) \\
982(44) \\
2177(64) \\
1268(60) \\
10922(67) \\
10540(55) \\
9158(63) \\
10918(24) \\
568(8) \\
\end{array}$ & $\begin{array}{l}2458(20) \\
2554(20) \\
2215(20) \\
2092(30) \\
3054(30) \\
2681(30) \\
1902(23) \\
2253(23) \\
2823(23) \\
3404(10) \\
3682(10) \\
4274(10) \\
6477(8) \\
6143(8) \\
3758(4) \\
2460(4) \\
4373(4) \\
3894(4) \\
1217(4) \\
1152(4) \\
1302(4) \\
4682(5) \\
3917(5) \\
3943(5) \\
5075(4) \\
5705(4) \\
5807(3) \\
6102(3) \\
4980(3) \\
2496(3) \\
2809(5) \\
3360(5) \\
4712(15) \\
5363(15) \\
4207(4) \\
2053(4) \\
6127(38) \\
3891(35) \\
3012(49) \\
5204(6) \\
9068(4) \\
6544(9) \\
5296(5) \\
8922(74) \\
2544(55) \\
\text { (5) }\end{array}$ & $\begin{array}{l}299 \\
299 \\
247 \\
380 \\
380 \\
380 \\
333 \\
333 \\
333 \\
268 \\
268 \\
268 \\
126 \\
126 \\
61 \\
72 \\
72 \\
72 \\
126 \\
126 \\
126 \\
114 \\
114 \\
114 \\
76 \\
75 \\
49 \\
48 \\
55 \\
70 \\
128 \\
128 \\
328 \\
328 \\
89 \\
82 \\
139 \\
202 \\
156 \\
140 \\
129 \\
149 \\
131 \\
136 \\
119 \\
\end{array}$ \\
\hline
\end{tabular}




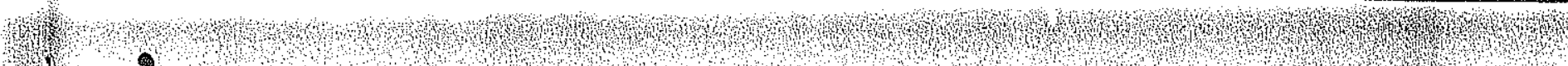

70011.

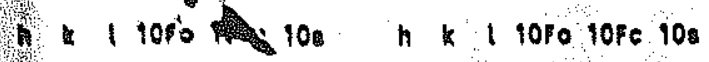

8. $0 \quad 0$ 461 470 15

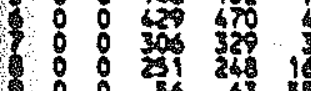
19.

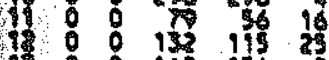

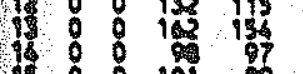
+1 8 8 101 \%

19.10019010011

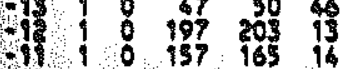

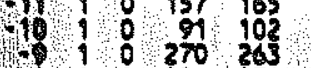

0.270
0
0

$-10023219$

$-3 \quad 0.85358$

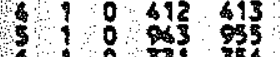

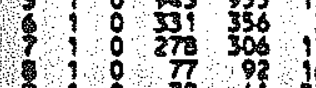

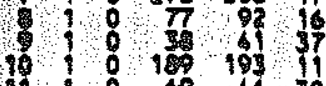

11.00090

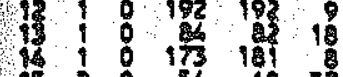

$\begin{array}{llllll}14 & 1 & 0 & 17 & 181 & \\ 3 & 0 & 5 & 0 & 57\end{array}$

-1320919 90 14

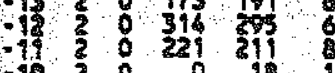

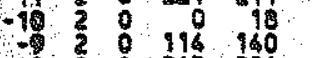

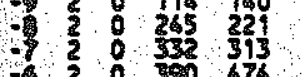

$-9{ }^{2} 012021226$

- 20 10 11 20

3 2 0 of 10352

$3 y^{2} \quad 972 \quad 503$

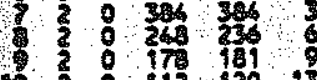

$102 \quad 912 \quad 42013$

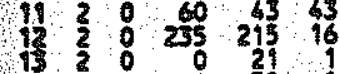

$\begin{array}{rrrrrr}14 & 2 & 0 & 0 & \frac{2}{38} & 1 \\ & 3 & 0 & 61 & 59 & 39\end{array}$

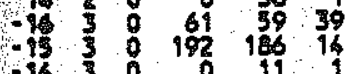

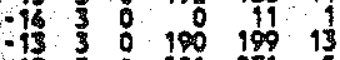

$\begin{array}{rrrrrr}-12 & 3 & 0 & 281 & 271 & 5 \\ -11 & 3 & 0 & 241 & 223 & 10\end{array}$

$\begin{array}{llllll}-11 & 3 & 0 & 261 & 225 & 10 \\ -10 & 3 & 0 & 287 & 297 & 5\end{array}$

$\begin{array}{lllll}-9 & 3 & 0 & 226 & 221 \\ -9 & 3 & 0 & 535 & 556 \\ -7 & 3 & 0 & 577 & 581\end{array}$

$\begin{array}{lllll}-7 & 3 & 0 & 577 & 581 \\ -6 & 5 & 0 & 230 & 230\end{array}$

$\begin{array}{lllll}-5 & 3 & 0 & 248 & 806 \\ -3 & 3 & 0 & 388 & 377\end{array}$

$\begin{array}{llllll}1 & 3 & 0 & 1197 & 1102 & 4 \\ 2 & 3 & 0 & 1160 & 1096 & 26\end{array}$

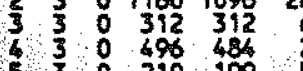

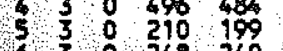

$\begin{array}{lllll}63 & 0 & 248 & 240 & 4\end{array}$

8 उ 3 : $176 \quad 159$ 22

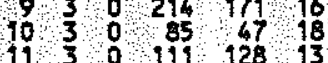

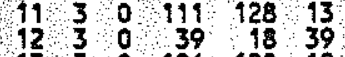

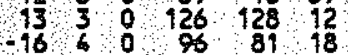

$\begin{array}{rlll}-16 & 0 & 9 & 318\end{array}$

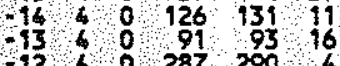

$\begin{array}{rlllll}-12 & 0 & 0 & 287 & 290 & 4 \\ 4 & 0 & 33 & 449 & 3\end{array}$

- 11550221210.20

-17.70
16,7

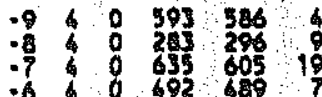

- 1049169

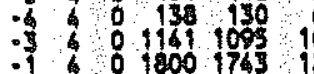

0 o 0216

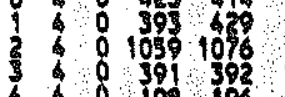

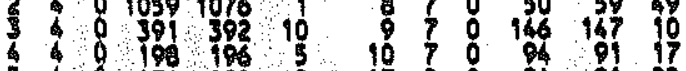

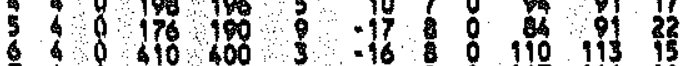

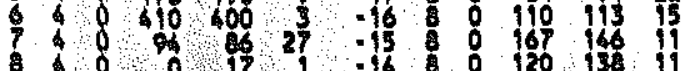

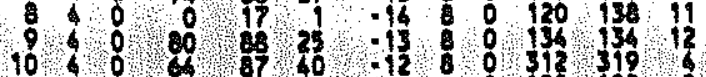

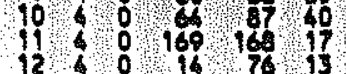

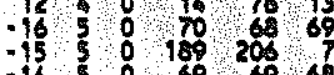

-16 506960

- 13 o 0108

- 5 5 0 \% 969

-9
-7

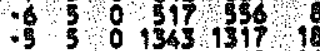

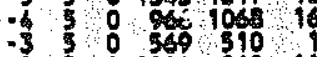

- $\begin{aligned} & 0 \\ & -100\end{aligned}$

- 50 o 072

$350.46 \%$

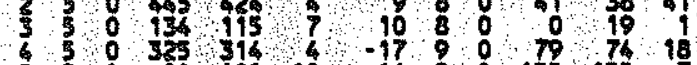

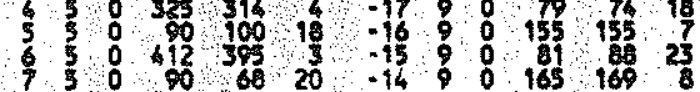

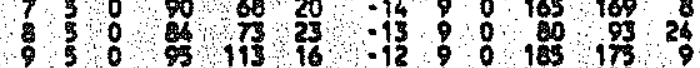

10 ह 019200 \&

-17 50 . 5191917

-15:8. 109100100

$\begin{array}{llllll}5 & 6 & 0 & 50 & 30 & 59\end{array}$

$-126081898$

$-11.60170198$

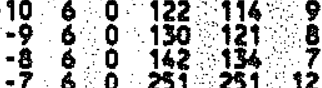

$\begin{array}{llll}-7 & 6 & 0 & 251 \\ -6 & 6 & 0\end{array}$

- 60 o 76874

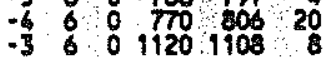

-2 $6 \quad 0102309011$

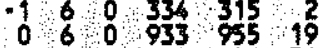

1 $601419,1396 \quad 36$

$260 \quad 548 \quad 530 \quad 5$

$3.0 \quad 449 \quad 256 \quad 9$
$6 \quad 0 \quad 277 \quad 271$

$\begin{array}{llllll}5 & 6 & 0 & 0 & 48 & 1 \\ 6 & 6 & 0 & 88 & 94 & 14 \\ 7 & 6 & 0 & 56 & 93 & 55\end{array}$

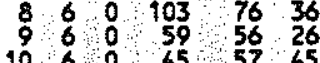

$10 \quad 6 \quad 0 \quad 45 \quad 57 \quad 45$

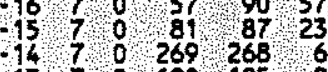

$-147 \% 0$ 269 268 136

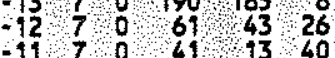

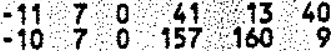

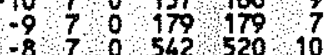

870054252010

$-6770 \quad 419,426,3$

$\begin{array}{lllll}-5 & 7 & 0 & 647 \\ -3 & 7 & 0 & 605 & 0\end{array}$

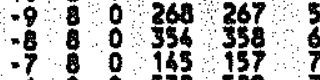

$\begin{array}{lllll}-16 & 10 & 0 & 103 & 60 \\ -15 & 10 & 0 & 42 & 16\end{array}$

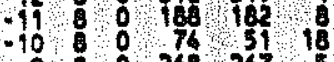

-6 80535297

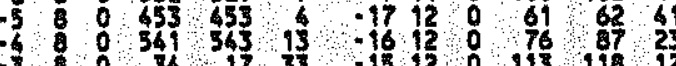

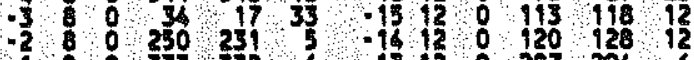

$\begin{array}{lll}1 & 0\end{array}$

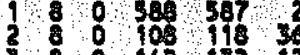

$6 \quad 0 \quad 142 \quad 132 \quad 12$

$\frac{6}{7} 8$ 111 100 26

- 1201201314

$\begin{array}{llllll}-612 & 0 & 240 & 261 & 7 \\ -7 & 12 & 0 & 190 & 100 & 9\end{array}$

$\begin{array}{llllll}-6 & 12 & 0 & 74 & 66 & 25 \\ -5 & 12 & 0 & 190 & 163 & 5\end{array}$

-3 12 0 126 125 12

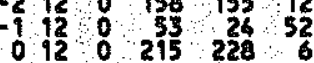

$\begin{array}{llll}1 & 0 & 215 & 228 \\ 1 & 0 & 255 & 262\end{array}$

312009015199

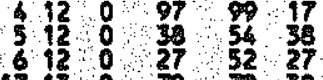

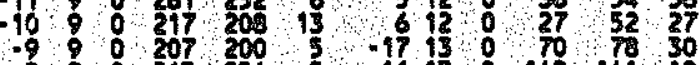

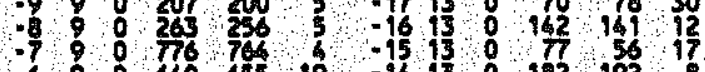

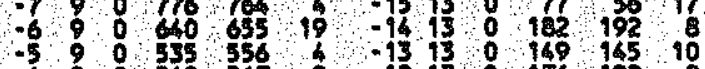

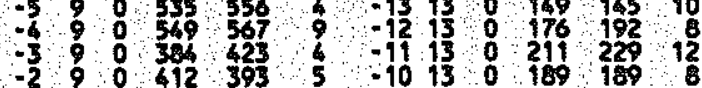

$-5 \quad 9 \quad 0 \quad 412 \quad 393$

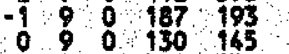

$\begin{array}{llll}1 & 0 & 325 & 359\end{array}$

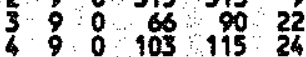

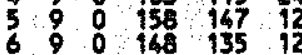

790012210818 890 95 81 it

-1710 o $142{ }^{2} 13412$

$\begin{array}{rrrrrr}-15 & 10 & 0 & 42 & 52 & 41 \\ -14 & 10 & 0 & 165 & 173 & 8\end{array}$

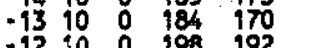

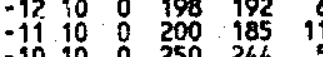

$\begin{array}{rrrrrr}-10 & 10 & 0 & 250 & 264 & 5 \\ -9 & 10 & 0 & 256 & 270 & 8\end{array}$

$\begin{array}{lllll}-8 & 10 & 0 & 334 & 316 \\ -7 & 10 & 0 & 332 & 343\end{array}$

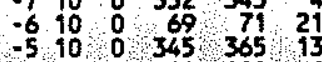

$\begin{array}{lllll}4 & 10 & 0 & 199 & 188 \\ -3 & 10 & 0 & 387\end{array}$

$\begin{array}{lllll}3 & 10 & 0 & 387 & 404\end{array}$

$-1100350,337,6$

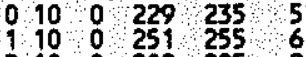

$\begin{array}{lllll}2 & 10 & 0 & 209 & 225\end{array}$

410 0 180 163 10

$51000 \% 989328$

6100 163 16311

$=9130$ 241 232

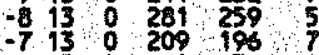

$\begin{array}{lllllllll}-6 & 13 & 0 & 0 & 34 & 1 \\ -5 & 13 & 0 & 164 & 154 & 16\end{array}$

$\begin{array}{lllllll}-5 & 13 & 0 & 164 & 154 & 16 \\ -4 & 13 & 0 & 168 & 150 & 22\end{array}$

$\begin{array}{llllll}-3 & 13 & 0 & 204 & 189 & 7 \\ -2 & 13 & 0 & 143 & 158 & 24\end{array}$

$\begin{array}{llllllllll}-1 & 15 & 0 & 117 & 145 & 29\end{array}$

$\begin{array}{lllll}013 & 0 & 265 & 268 & 10 \\ 113 & 0 & 239 & 260 & 7\end{array}$

$\begin{array}{lllll}2 & 13 & 0 & 0 & 26\end{array}$

$\frac{3}{6} \quad \begin{array}{lllll}13 & 0 & 65 & 61\end{array}$

$\begin{array}{lllll}513 & 0 & 46 & 18 & 45\end{array}$

$\begin{array}{lllll}-1614 & 0 & 135 & 119 & 15\end{array}$

$\begin{array}{lllll}-1514 & 0 & 0 & 42 & 1\end{array}$

$\begin{array}{rrrrr}-1314 & 0 & 234 & 226 & 6\end{array}$

$\begin{array}{lllll}-12 & 14 & 0 & 58 & 36 \\ -11 & 58\end{array}$

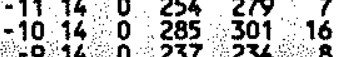

\begin{tabular}{lllll}
-914 & 0 & 237 & 234 & 8 \\
\hline & 14 & 0 & 314 & 303
\end{tabular}

$-714,0,135,122,9$

-544 o 224 19,

-414 o 91 प8 45

$\begin{array}{llllll}-3 & 14 & 0 & 254 & 254 & \\ -2 & 14 & 0 & 200 & 187 & 11\end{array}$

$-114 \quad 0 \quad 104 \quad 134 \quad 22$

$\begin{array}{lllll}0 & 14 & 0 & 221 & 225 \\ 1 & 14 & 0 & 144 & 150 \\ 2 & 14 & 0 & 125 & 137\end{array}$

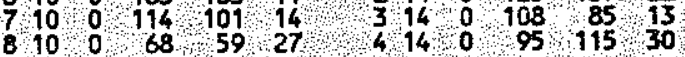

h $10 \% 10 \mathrm{Fo} 10$

$-1619000018$

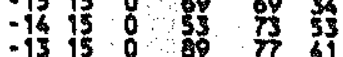

.1215 o 191.138 iा

- $1015 \quad 0 \quad 90 \quad \frac{139}{75} 24$

-8 is 0 264 238 16

$\begin{array}{llllll}-7 & 15 & 0 & 259 & 278 & 6 \\ .6 & 15 & 0 & 3 & 350 & 6\end{array}$

-515007 16 19

.3 is 01521429

- 15 ono 49

1150 12 196217

-1616\% 152 160 22

-16 160 05046

-131600911822
-12.60

-11.160 o 152,150

1016 0 114 102 12

- 160226216

- 716 0 2913 302

- 1610203208

- 16 on 115 log 14

-2 $160055 \quad 5538$

0160 0 42

$\begin{array}{rllll}1 & 16 & 0 & 8 & 32 \\ -15 & 17 & 0 & 38 & 64\end{array}$

- 1317 o 108 191 18

-12170106 , 15

- 1017 0 120 133 22

- 170 100 142 11

$\begin{array}{llll}-7 & 17 & 2010195 & 12\end{array}$

$\begin{array}{llll}-610 & 108 & 92 & 18\end{array}$

$-\$ 170$ a 16016512

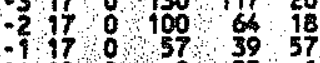

$-14180+0055$

-15180.129 161 15

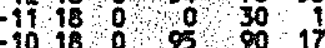

-9 18 0 168 161 11

- $18005150 \quad 50$

$-618026 \quad 46 \quad 26$

$\begin{array}{llllll}-5 & 18 & 0 & 64 & 68 & 37 \\ -4 & 18 & 0 & 32 & 29\end{array}$

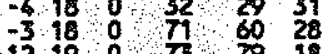

-121907379 18

$\begin{array}{lllll}-10 & 19 & 0 & 45 & 37\end{array}$

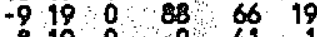

$\begin{array}{llllll}-8 & 19 & 0 & 0 & 41 \\ -7 & 19 & 0 & 36 & 51 & 35\end{array}$

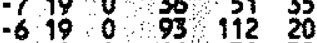

$\begin{array}{lllllll}-519 & 0 & 66 & 30 & 32\end{array}$

$\begin{array}{lll}4-19 \quad 1 \quad 61 & 35 & 42\end{array}$

$6-19176 \quad 8021$

$8-191009$

$9-191104$ ए 7919

$\begin{array}{llll}10-19 & 26 & 58 & 26\end{array}$

$12-19,118063$

$2-18,170 \% 7$

$3-18.1$ 118, 109,16

$5-181,55$ 82 54

$6-181190$ 199 21

$7-181+76$ 105 76

$8-181 ? 55,94 \quad 36$

$10-181276 \quad 92 \quad 20$

$11-18,170032$

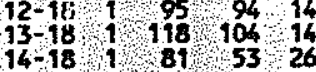


.

PQ

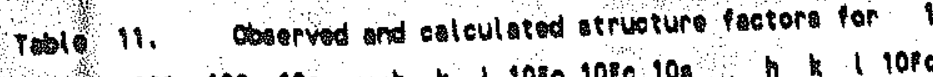

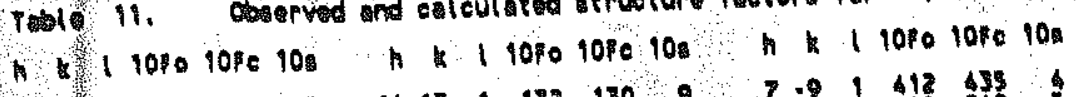
0.17 10 25

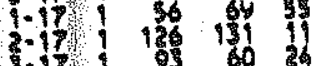
3.17, 93190 3.17\% 2835 . 3.17 0 की 10-17\% 15 140 $11.171 \%$ 121 14 $13-17$ 1 33 39 35

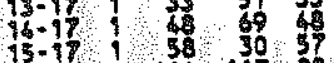
$15-71,158$
-2.161972
-19 +1.96101 19 30

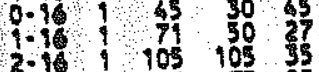
$\begin{array}{lll}3.1 & 105 & 105 \\ 73 & 75 \\ 130 & 116\end{array}$

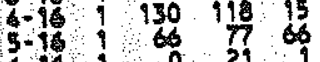

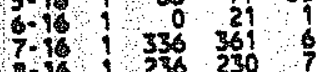
$\begin{array}{lll}9-16 & 236 & 30 \\ 2 & 0\end{array}$ $10-19 \quad 2692515$ $12-10,169$ 171 11 $16-861$ 4 1696

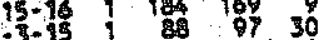
$-2-15$ 9 113 130 13

$-0-15$ i 155 in 19

2-15 $10^{5}-1276^{4}$

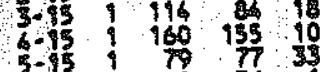

015
$7-65$ 190976

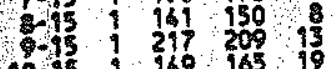

$10-95$ 1 149 165 19

$12-75$
$13-15$$\quad 213 \quad 222 \quad 10$

18-35 6527

15-15 1062

$\begin{array}{lll}-8-16 & 0 \\ -3-16 & 28\end{array}$

$-2-16,947617$

-156 1 253 251

214 $1314227 \quad 9239$

3it 190910516

9. I6 236 212

$614 \quad 264237 \quad 6$

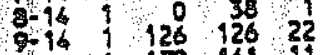

$10-16,172 \quad 169$ 212

$\begin{array}{llll}11.14 & 1 & 208 & 212 \\ 12-14 & 29 & 56 & 28\end{array}$

$\begin{array}{ccccc}13-16 & 1 & 190 & 984 & 7 \\ 10-16 & 1 & 184 & 179 & 16\end{array}$

15-14 16 i6 16

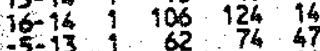

$-6-13+1421381$

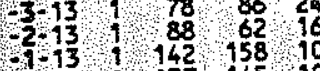

$\begin{array}{lll}1-13 & 13\end{array} 137,145$ का 10

$2-13$ । 299 319

$\begin{array}{lllll}3 & -13 & 1 & 336 & 327\end{array}$

$\begin{array}{llll}5-13 & 116 \quad 10813\end{array}$

$\begin{array}{lllll}6-13 & 1 & 167 & 139 & 9\end{array}$

$8-13 \quad 1241 \quad 23912$

9.13 $1231 \quad 226 \%$

10-13 $1161 \quad 161 \% 9$

$\begin{array}{lllllll}19 & 13 & 163 & 146 & 10 \\ 12 & 13 & 1 & 264 & 258 & 7\end{array}$

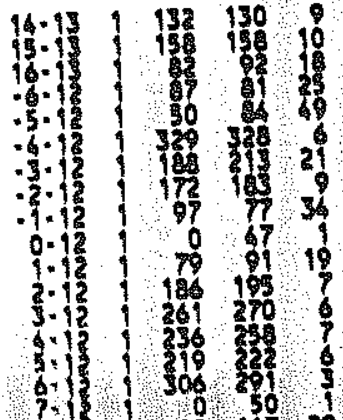

ब. 1 1 100,117 ,

$10 \cdot 12,4003013$

$11-12,209210$

$13 \cdot 12,184 \%$

$16.12 ! 25,563$

$-7.11,1$ दी 48 , 52

- $5.119196,16410$

- $-1919 \frac{257}{75} 157$ ?

$0.11 \quad 656625$

1:11 1250 250 10

3.11 .269231

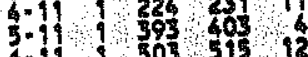

7.11 1110108

9.1 113616 .

$11-11.258271$ \%

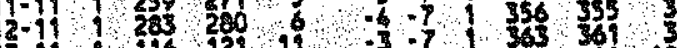

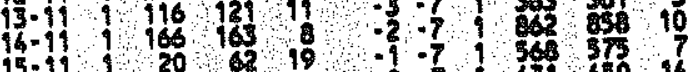

$15.120 \% 19$

$17-11,180 \% 22$

$-8=10,5639$

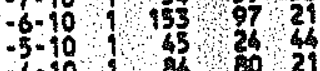

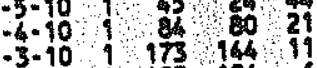

$-2+10,193196$

$-1.10,1342,352$

$0-10$
$1-10,312$ 38 ?

$\begin{array}{llll}2.10 & 307 & 322 & 6\end{array}$

$410,61,5520$

$6-10 \quad 673470$

$7-10 \quad 435402$

9.10 1 165156

10.10 : $154 \quad 131,7$

$12=10+134,130,12$

$13-10$
$14-10$ $128121 \quad 13$

\begin{tabular}{l|ll}
$16-10$ & 134 & 126 \\
$15-10$ & 127 & 126
\end{tabular}

$\begin{array}{lll}16-10 & 1 & 99 \\ 17-10 & 61 & 16\end{array}$

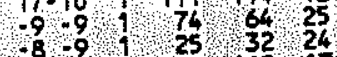
79914 19. 17 $6.9,1181$ 257 24517 $-40,1,137$ 158 11 $-3-9,1208206$ ? -2.91100 \%2,14

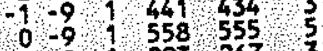
$1.9,1,293,267$ ?

$2,9,1216,213,49$

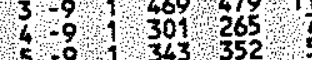

5.9.1, 343,352
8-11 196176,10

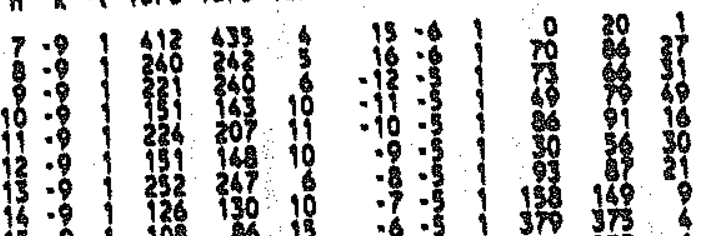

$13=108$ \%

19.9019

-9.8 1150 14

- 10171010

- 6.6117810

- -6113260

0.8135

$2=10671049$

$3-8,160 \mathrm{~T}$

8.81263 25

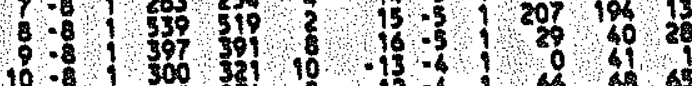

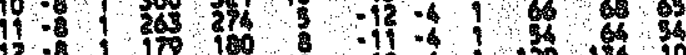

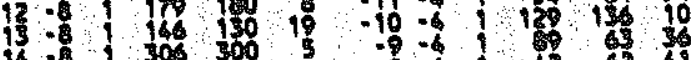

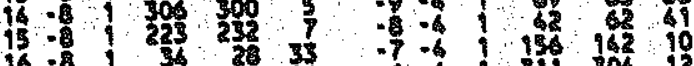

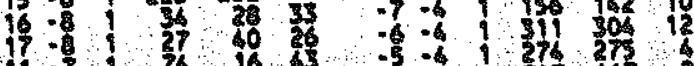

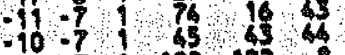

$-9-7,182182$.

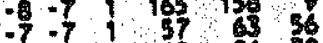

-6 -7 $\quad 02 \quad 30$

$1-7 \quad 212$

$3-7,10051011,12$

61009

$\begin{array}{lll}5-7 & 105 & 517 \\ 6 & -7 & 447\end{array}$

7.7129328

$8-7 \quad 4765515$

$\begin{array}{llll}9 & -7 & 522 & 52 \\ 0 & -7 & 132\end{array}$

$\begin{array}{lll}11 & -7 & 243224 \\ 12-7 & 104\end{array}$

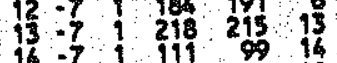

$15-7,12914410$

$\begin{array}{rrr}16 & -6 & 1\end{array} 9129624$

$-10-6 \quad 100453$

$\begin{array}{llllll}-9 & -6 & 1 & 121 & 123 & 13 \\ -8 & -6 & 289 & 27 & 5\end{array}$

$-7.616555: 38$

$\begin{array}{llllll}-6 & -6 & 1 & 266 & 259 & 13 \\ .5 & -6 & 1 & 430 & 427 & 10\end{array}$

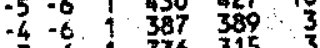

$-3-61336 \quad 315$

$\begin{array}{llll}-2 & -6 & 1 & 369 \\ -1 & -6 & 1 & 605\end{array}$

166,567 542,

2.6 1170 रा 11

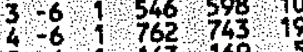

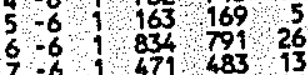

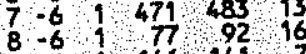

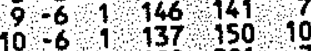

$116,6199,2019,7$

$12.6,172,39,31$

136,1419199612

$12-3$. 152

13.517879

$-6-6,170^{2}{ }^{2}$

$-3-127535$

- $-4 \quad 040$ h 2 1 10FA 16\% 108

-3

\%. 15

7.2 37 196

$10.2,5515 \%$

11.51200

1

$15: 2$ 13 39

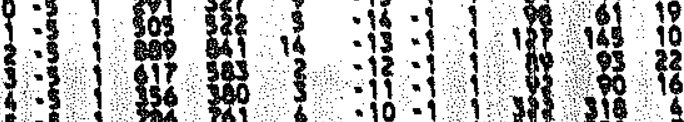

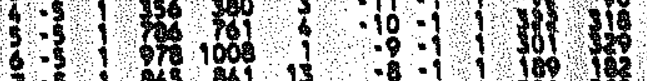

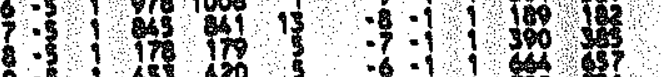

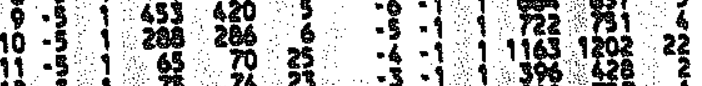

3. 74306

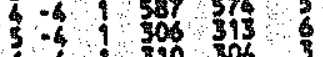

7.613702010

$7-6.508525$

$10-4137$ is 8

$12-51306325 \%$

$13-2177 \pi 7$

$\begin{array}{llll}14 & -4 & 0 & 22 \\ 15 & -4 & 58\end{array}$

$16-6 \quad 1008223$

$\begin{array}{lllll}-13 & -5 & 122 & 112 & 16\end{array}$

$\begin{array}{llll}-12 & -3 & 200 \quad 201 \\ -11 & -3 & 6\end{array}$

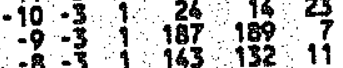

$\begin{array}{lllll}-8 & -5 & 163 & 132 & 11 \\ -7 & -3 & 92 & 8 & 17\end{array}$

$-6-3 \quad 576563$

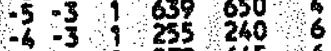

-3.3 15786596

\begin{tabular}{rr|rrr}
-2 & -5 & 810 & 826 & 95 \\
-1 & -3 & 1076 & 1066 & 1 \\
6 & -3 & 1739 & 1655 & 17
\end{tabular}

$\begin{array}{llllll}5 & -3 & 601 & 581 & 1 \\ 6 & -3 & 347 & 345 & 5\end{array}$

\begin{tabular}{ll|lll}
$6-3$ & 1 & 347 & 345 & 5
\end{tabular}

$\begin{array}{lllll}7 & -3 & 465 & 590 & 11 \\ 8 & -3 & 565 & 597 & 4\end{array}$

$9-3 \quad 146$ 144 8

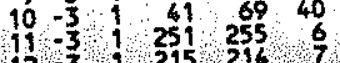

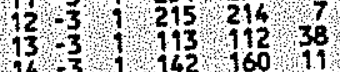

$14=3,19142,160$. 11

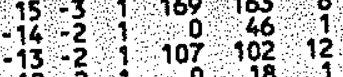

$3-11979375$

$5-1,165191,10$

6.1169515

-1 153048

$11=1-201$ is

$13-1 \quad 100$ \% 19

is 15109164 i?

- 1301301269

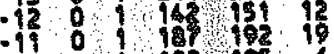

-10 0 1019

$-70153327$

-6 0 310 $535 \%$

-5 0 225 $226 \quad \frac{5}{35}$

\$ 0.62761916

5 0168193

$601300 \%$

80142150720

9 0125025716

11012835 ?

$\begin{array}{lllll}15 & 0 & 116 & 121 & 12 \\ 14 & 0 & 164 & 158 & 8\end{array}$

$-1511231072$

$\begin{array}{lll}-13 & 137 & 25 \\ 157 & 20\end{array}$

-11 $107 \%$

- $10 \quad 1100 \%$

$-9111491469$

$-7 \quad 1359 \quad 341$

$\begin{array}{lll}-6 & 1619603 & 2\end{array}$

- $1 \frac{537}{750} 706$

31.4684097

$511336 \quad 351 \quad 13$

6. 11176 171 6

$8.11735,424,7$

$101,1128,9612$

$12,121,150$ \% 1449

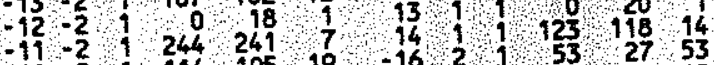

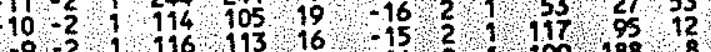

$\begin{array}{lllllllllll}-8 & -2 & 144 & 199 & 21 & -14 & 2 & 199 & 188 & 8\end{array}$

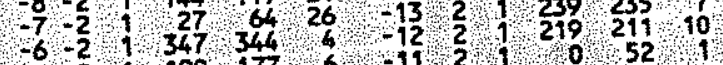










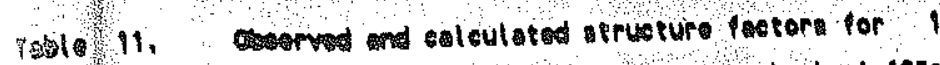

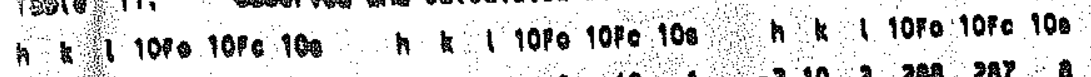

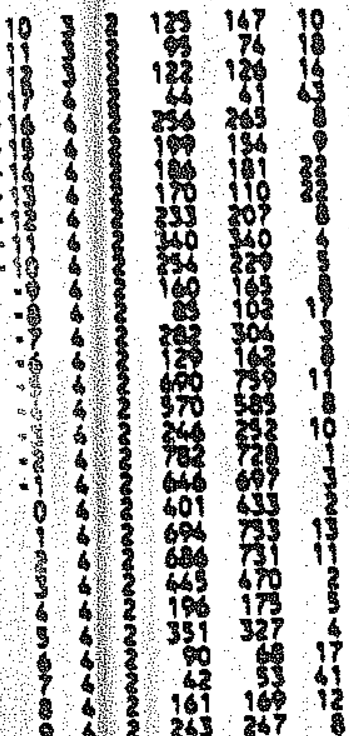

1073

-115 19

- $19.5 \%$

-19 10 . 13

-19.0.

$-7.150$

- I I1 \$

-1) 19

$10 \%$

31519

31 भी 17

1. 200

10 \% 109

$170 \quad 1490210$

-1516 100 1092

.130 2 120 15 12

.1916 2 25 245 11

-9 6 19

- 70 ह 3519

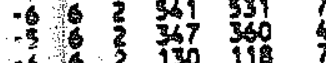

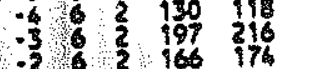

$\begin{array}{llll}-2 & 6 & 2 & 166 \\ 0 & 6 & 2 & 179\end{array}$

2. 6 6 ? 360 , 382

36255.53 . 3

662252234

$6162,137,137$

6. 6 2 185 , 95

$10162+165$, 160,10

$1977 \%$ ? $126 \quad 63$, 26

$1657,2,106,95,33$

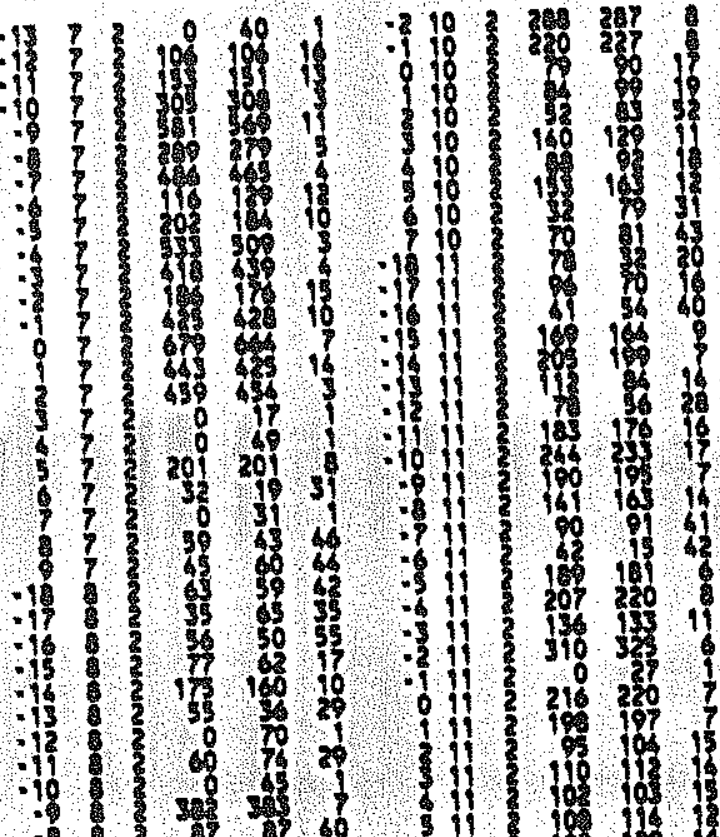

-1) 10

- 516

- 15

- 21,15

2 1010

3 मी

7.

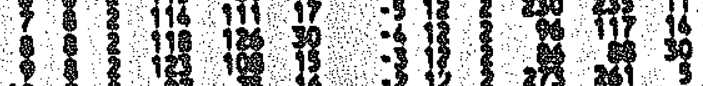

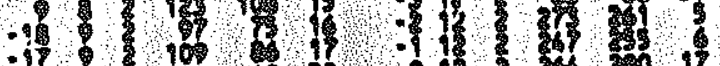

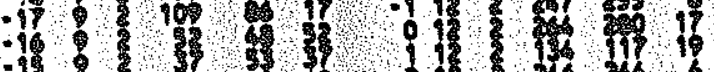

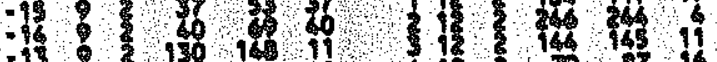

-12 10171

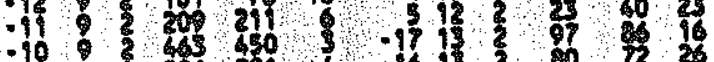

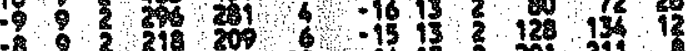

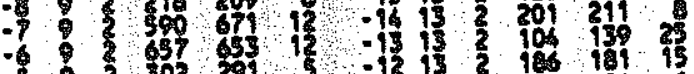

- $52 \quad 30281$

.4251116

-2 9220262

09176,164

301902008

$\begin{array}{lllll}3 & 2 & 104 & 139 & 18 \\ 4 & 2 & 33 & 33\end{array}$

52 2 3527

$\begin{array}{lll}6 & 2 & 36 \\ 7 & 100 & 26\end{array}$

$\begin{array}{rlll}8 & 2 & 0 & 25 \\ -1810 & 28\end{array}$

1710 ? 130,160 , 11

15102 2 1531510

1610 ? 28427310

-1210 2 $264,245,22$

-1010 ? 215 211 20

$\begin{array}{rl}-810 & 2399,388 \\ -7 & 210\end{array}$

$-610,2242248$.

-510 ? 1762 174.
- IJ n 11010 10\%o 100

- 15 o 19

114 of

.17 i 1

-11 137 167 15

-1 1 I I I

-15 1 100 11

- I का ।

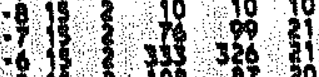

31321030

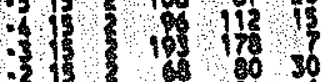

- 1300

113 10 169

-1616 \% 67

-16 12 121

1616 क 60

- 10 का 12

- 16 I10 210

- 1 151

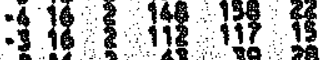

$-16 \% 00$

-15 19 15169

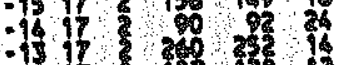

- 11 का का का

- 17,200 os

-1 17 का11

- 17 15 15

- 17 10 110

- 17 क 97 is

-19 18 o

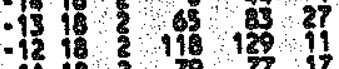

$.111827 \% 17$

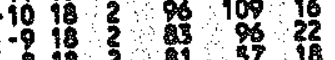

$\begin{array}{llll}-7 & 10 & 81 & 97\end{array}$

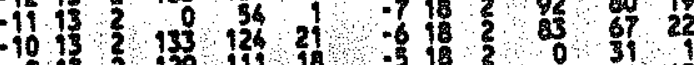

- 13212111

-8 $13 \quad 2 \quad 00 \%$

- 6132 20 1976

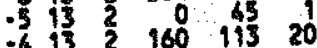

$\begin{array}{llllll}-5 & 3 & 2 & 51 & 82 & 50 \\ -3 & 13 & 63 & 105 & 62\end{array}$

$\begin{array}{lllll}-2 & 13 & 2 & 63 & 105 \\ -1 & 13 & 2 & 273 & 278\end{array}$

$013 \quad 2 \quad 121 \quad 116 \quad 14$

$\begin{array}{lllll}1 & 13 & 203 & 103 & 27 \\ 3 & 13 & 56 & 66 & 32\end{array}$

613 $3,67,60,30$

1714 ? 80,8027

-1512 2 14914512

-1314 2 $293,296,0$

-11162 , 4233 $012,245,236,13$ 814 ? $154 \quad 15411$

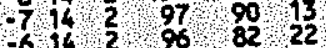
$614,2,96,134,13$

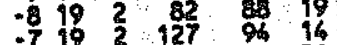

$3-19 \quad 3 \quad 79 \quad 9651$

$\begin{array}{lll}5-19 & 3 & 82\end{array}$

$\begin{array}{ccc}7.19 & 3 \\ 8.19 & 3 & 0 \\ 0 & 30\end{array}$

919.106 . 62 2 18

10.19 3 86 . 18

$8-183,5511202$
- 108 . 1080109

$19=180^{\circ} \mathrm{ol}^{\circ}$

1.

2.16 का 40

๕.17 10 हा

5.17 195

-17 197010

3. 101010

$10=17$ og 15

11-17 9010

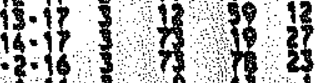

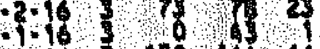

0.16 lis $^{16} 16$

2.16 ग 54

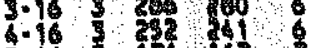

5.16 176 16215

7.16 \% 72

8.16 190165

$10.161151511 \%$

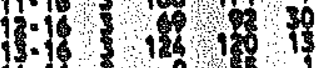

16.10103

- 1512100

-. .15 का

0.15352

2.15 1010,10

- 15100117

5.15273075

8. 13101

- 101111

$10-1$ \% of 29

11.13 ।

$14-15$ का की

15.15 32 का 15

- -143 o 95

- -14 - 126 12 17

- 16 10 12215

i-1 3503

2.16
$3-14$ 38016212

4. 14 3 190200 ?

3.16 3 85 25 26

7.14 रा8 111 15

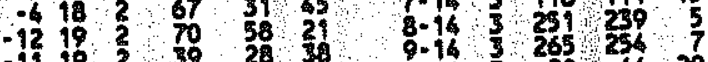

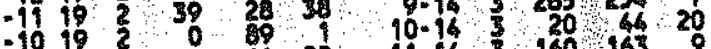

$\begin{array}{llllllllll}-9 & 19 & 2 & 23 & 61 & 22 & 11-16 & 3 & 2001612 & 7\end{array}$

6.193515751

$\begin{array}{llllll}178 & 3 & 0 & 41 & 1 \\ 2 & -18 & 43 & 55 & 43\end{array}$

$3-183,82,51,21$

$5-18,3,94,75,36$

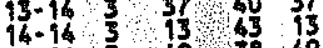

$15-16 \quad 3 \quad 69 \quad 38 \quad 49$

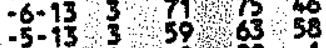

$-6-13,3, \quad 09128,25$

$-2-13,196201$.

$-113,1897130,12$

$1.13,160.189 .8$

$2.13,3,209,194,39$

$4-13,48$, 65,67

$6-133$. 51 ,

$\begin{array}{lllll}6-18 & 3 & 54 & 44 & 54 \\ 7-18 & 100 & 61\end{array}$

$713,3,17,163,7$

$10-18 \quad 3 \quad 124$ प 42 . 10

$\begin{array}{ccccc}9-13 & 3 & 185 & 180 & 14 \\ 10-13 & 3 & 124 & 19 & 13\end{array}$ 
1610 11, orved calculated structure factor for 1

T. 1100 iofo 108

$11 \cdot 13$ 3 1319523

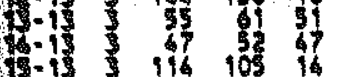

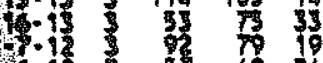

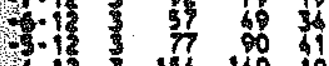

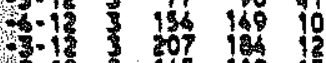

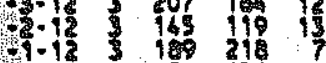

$0.12 \quad 3 \quad 171$ 151 11

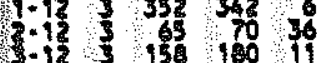

$\begin{array}{lll}-1 & 3 \\ -15 & 3 & 30\end{array}$

5.12 $34 \quad 210$

Q.12 2020

10.12 32720

$17.12 \quad 3$ रा 3

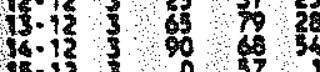

55.12 3 39 37 39

8.11 16917

- 6.113176 if

$5-193235$

E.11 376270

$4.11 \quad 112110$.

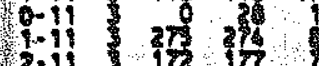

2.11 1701797

1.11 क5 65 7

-11 29525

- I1 19010

10-11 22820

11.11 $199^{9} 100$

13.1 12 121

15.11 71 23

19-11 71

-8.10 भा 11130

7.10 \% 100

$6-10$
$5-10 \quad 36123$

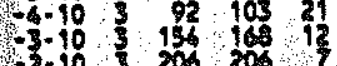

2.10 260306

$0.10 \quad 200262$.

$1.10 \quad 262 \quad 274$ \%

8.10
$3.90 \quad 30519$
6.10

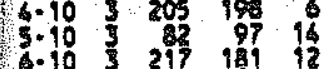

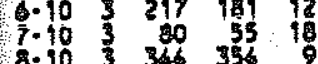

$\begin{array}{llll}8.10 & 3 & 346 & 354 \\ 9-10 & 3 & 173 & 131\end{array}$

$10-10$
110

$92-10$ 3 66 ह9 20

1310 3 132.165 . 18

$16-10.3496$, 9511

$\begin{array}{cccc}16 \cdot 10 & 3 & 98 & 98 \\ 90 & -9 & 109 & 87\end{array}$

$\begin{array}{ll}-9 & 9 \\ 3 & 53 \\ \end{array} \quad 76 \quad 33$

$7.93188 \quad 177$ \&

60930471

$\begin{array}{cccc}5.9 & 324 & 115 & 19\end{array}$

$-309 \quad 78 \quad 6619$

$\begin{array}{lllll}-2 & 9 & 3 & 542 & 527 \\ -1 & 9 & 3 & 150 & 138\end{array}$

$\begin{array}{llllll}0 & 9 & 3 & 551 & 564 & 7 \\ 1.9 & 3 & 392 & 404 & 3\end{array}$

$2.9 \quad 3504500$

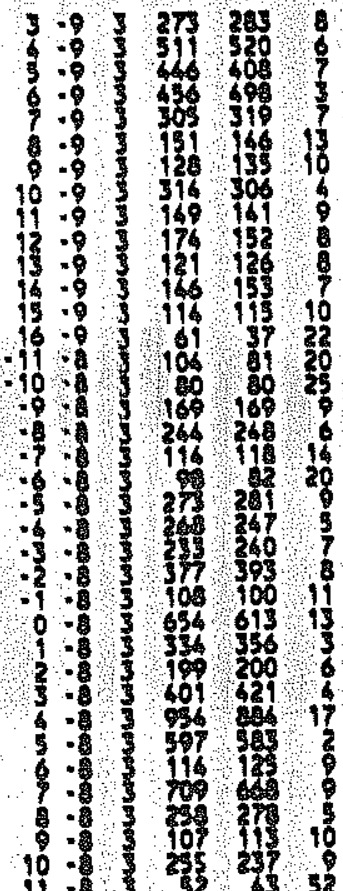

10.5253

$13-1010$ रा

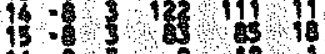

$16-910019$

-11 $-7 \quad 101309$

$\begin{array}{ccc}-9 & -7 & 203 \\ -8 & -7 & 169\end{array}$

$\begin{array}{ccc}-7 & -7 & 169\end{array}$

$-6-7 \quad 0033$

- $-7 \quad-7 \quad 17911612$

$-.713786$

0.7 s 696

$-7 \quad 00515$

$3.7 \quad 5805$

$-7 \quad 64617$

$\begin{array}{llll}3 & 7 & 3 & 27 \\ 8 & -7 & 382\end{array}$

$7.7 \quad 205205$

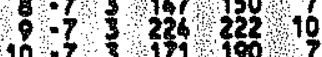

$11.7,3139,13629$

$\begin{array}{llll}12 & -7 & 3 & 63\end{array} 69$

$16-7 \quad 75 \quad 5920$

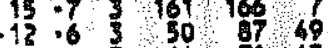

$\begin{array}{lllll}-11 & -6 & 3 & 3 & 71\end{array} 43$

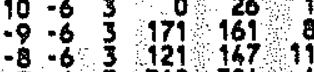

$-7.63319,306,6$

-5.6 31649372

$\begin{array}{lllll}-4 & -6 & 3 & 647 & 698 \\ 3 & 690\end{array}$

$\begin{array}{ccccc}-2 & -6 & 3 & 536 & 577 \\ -1 & -6 & 3 & 1056 & 1064\end{array}$

163 342 84 ।

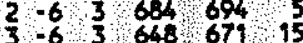

463 3 449651 ?

$5-6,3,4674477$ ?

6.634484 .459

$8.63,323192$

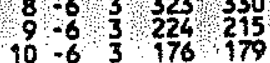

0.63 39 405 . 24

$\begin{array}{lllll}90 & -6 & 3 & 176 & 179\end{array}$ h $110 \% 0$ 10\% o 100

$12.6 \quad 202 \quad 192$

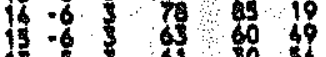

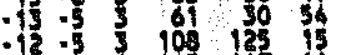

-12 -15310816713

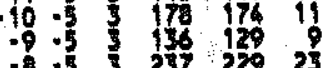

- $7.5 \quad 3 \quad 25 \quad 29025$

- 6 .5 218236

- -5335 35 3

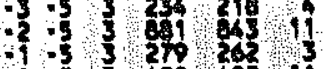

$03340 \%$ का

$3=5313000$

6 3 64016

$\begin{array}{llll}5 & 5 & 51 & 650 \\ 7 & 5 & 38\end{array}$

$\begin{array}{llll}7 & 5 & 5 & 28 \\ 8 & 5 & 3 & 223\end{array}$

$10-53135$ is 170

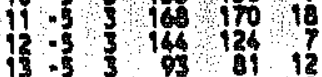

16.5169

- 14 \%

$-11=4 \quad 155160$

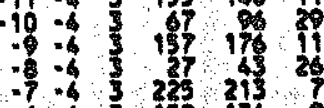

- 5 - 6 15 131

- 6 -4 53157 12

$-3-6130212311$

0.45319

2. 6145139

3.67617

3 - 12

6.3515

8 - 3 2 31

$10.4 \quad 112$ । 127

$\begin{array}{llll}11 & -6 & 3 & 129 \\ 3 & -4 & 35\end{array}$

$12-4 \quad 1271271$

$15-3 \quad 811910$

$\begin{array}{rrr}-16 & 3 \\ -13 & 3 & 02\end{array}$

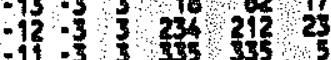

- 10 - $\quad 176 \quad 18010$

$\begin{array}{lllll}-9 & -3 & 3 & 239 & 218 \\ -8 & -5 & 3 & 212 & 218\end{array}$

$\begin{array}{llll}-7 & -5 & 5 & 212 \\ -7 & -5 & 356\end{array}$

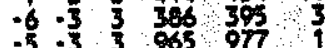

$\begin{array}{llllll}-4 & -3 & 3 & 602 & 648 & 8\end{array}$

$\begin{array}{llllll}-3 & -3 & 3 & 680 & 493 & 15\end{array}$

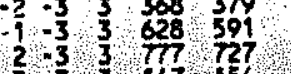

$\begin{array}{lllll}3 & -3 \\ -3 & 3 & 974 & 153\end{array}$

$5.3,3452,460$. 8

6.3
7 3 2731272910

$\begin{array}{llllll}8 & -3 & 3 & 135 & 116 & 7 \\ 9 & -3 & 3 & 136 & 149 & 15\end{array}$

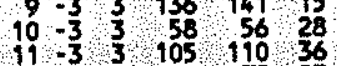

$\begin{array}{llllll}11 & -3 & 3 & 105 & 110 & 36\end{array}$

$\begin{array}{cccccc}13 & -3 & 3 & 160 & 153 & 7\end{array}$

h $110 \%$ 10\% 10

91.2129127

$10.3 \quad 1319$

-

- 0 2 1132

$-5=2 \quad 11481100$ \%

$-3.2 \quad 79073$

3.2 91685

1.2 40.07

\begin{tabular}{ll}
6.2 & 535 \\
\hline & 559
\end{tabular}

$6.2 \quad 169137$

$10-2$ 3 1315120

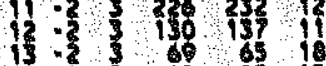

$15=3$ क

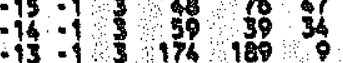

$12-13107160$

$10-135353515$

9.13536728

- - -1 318 317

-6 -1 573

$-13169509$

- -135451911

5.1 65 30 67

\% :-1 35543

-1 10 16 ?

$10-1 \quad 130$ I15

$12=1$ क 19

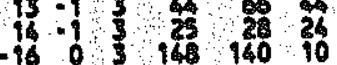

- 15001751751

$\begin{array}{llll}-16 & 0 \quad 120 & 130 & 13 \\ -13 & 0\end{array}$

-12 03 o 16

- $10.300 \%$

- 0327025018

-7035 311

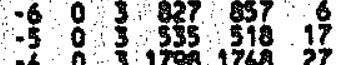

- $0 \quad 179$ 176 27

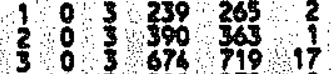

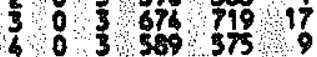

$50367 \quad 6212$

$60 \quad 450739$

$80 \quad 354026$

$90 \quad 3 \quad 11910411$

$1103 \quad 15191210$

$\begin{array}{lllll}12 & 0 & 3 & 39 & 38\end{array}$

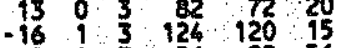

- 1513565

-14 $13240251 \% 5$

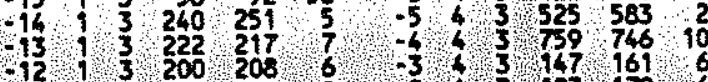

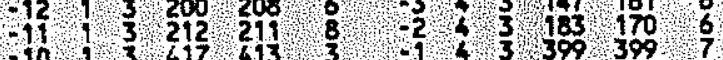

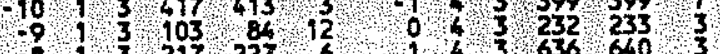

$\begin{array}{lllll}1.8 & 3 & 217 & 227 & 6 \\ -7 & 3 & 322 & 360\end{array}$

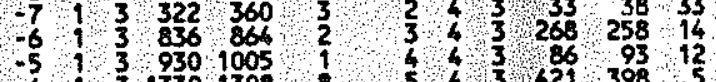

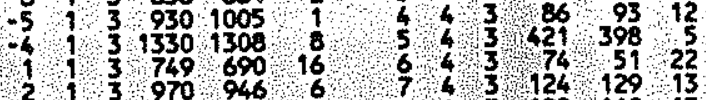

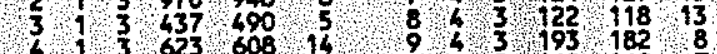

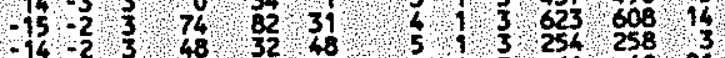

$\begin{array}{lllll}-14 & -2 & 3 & 48 & 32 \\ -13 & -2 & 3 & 48\end{array}$

$\begin{array}{lllll}-13 & -2 & 3 & 0 & 33 \\ -12 & -2 & 3 & 270 & 265\end{array}$ h 1 10Fo 10Fe 100

9010 का ?

$17 \quad 3 \quad 107$ so 51

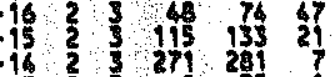

$12{ }^{2} \quad 0$ os 25

-10 2 37200

- 31010

3340

5231271207

723234

$\begin{array}{llll}2 & 3 & 65 & 16\end{array}$

12 2 $117 \quad 21190$

- 16 3 3 जा1 103 18

-13 3109 I07 17

- 5 J 395 302 is

-73) 353

13006

134056

4341641916

$73{ }^{126} \quad 1310$

10331521478

1233 ? 25

16,3148 136 13

करण21 1131

$11 \quad 33^{3}{ }^{53}{ }^{36}$

$\begin{array}{lll}-7 & 3 & 320\end{array}$

64342402 $943,193,162,8$

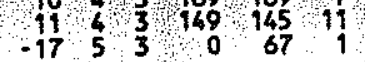

-72339 क 1717

31090

- 1606

-8 10303 
Tolo li. coorve and enloulated atructure fectore for 1

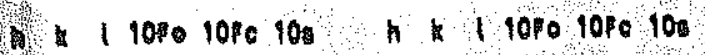

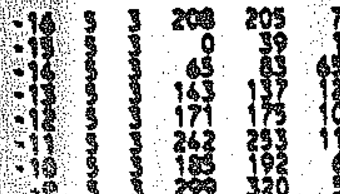

13 .

+3 105100

13503

15353250

$4316210 \%$

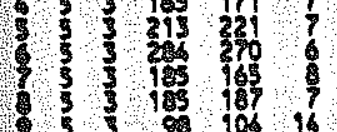

$10.35100 \%$

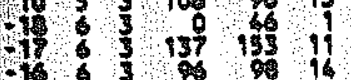

$113 \quad 179016$

-15 690

12 \% 50

-10. 1513015

1. 35 351

3 - 50100

$\begin{array}{ll}-3 & 507 \\ 350 & \end{array}$

1 $3 \quad 45364$

5 61001928

$5 \quad 340313$

Q $\quad 33$

1. 1919

-49 1096

$\begin{array}{llll}-17 & 7 & 3 & 0 \\ -7 & 7 & 3\end{array}$

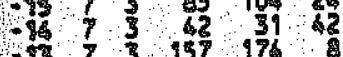

- $73 \quad 7 \quad 157 \quad 187$

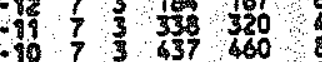

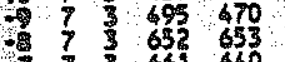

-7. 73 3 600

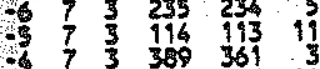

$\begin{array}{llllll}-1 & 7 & 3 & 389 & 361 & \\ 3 & 7 & 3 & 312 & 315 & 11\end{array}$

$\begin{array}{llllll}2 & 7 & 3 & 69 & 600 & 8 \\ 1 & 7 & 3 & 43 & 601 & 5\end{array}$

1. $73 \frac{162}{3} \quad 148$

$27,3 \quad 239222$

$4 \begin{array}{llll}4 & 3 & 163 & 147 \\ 7 & 3 & 126 & 10\end{array}$

$\begin{array}{lllll}67 & 7 & 74 & 62 & 26 \\ 7 & 50 & 99 & 42\end{array}$

$87,3,9011420$

$-1883,65061$

$178 \quad 3 \quad 119 \quad 109$ 38

$\begin{array}{lllll}15 & 8 & 3 & 205 & 199 \\ 8 & 98\end{array}$

$-1383245241,6$

$12 \quad 8 \quad 3 \quad 173 \quad 160,10$

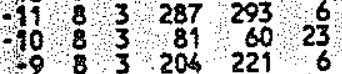

-11 10 16 167
- I 151 141 10

- 1019

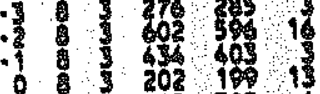

1 $\quad 202$ 105

3 1012

- 1191019

?.115 14741

$-179513513 \%$

-15.95150 14

-15 5141,304

.190273201

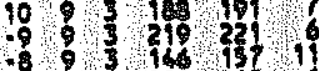

.8932202515

- 69540403

- 93510

- 31710

- 2 201

ร का हो।

3. 31191202

68351213 ?

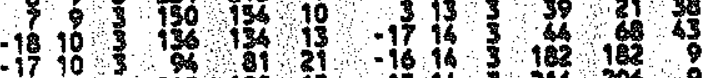

$-1610 \quad 3 \quad 205$ \% 198

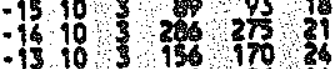

- 1210 , 150909

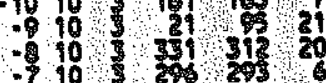

610 \% 20 .

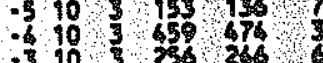

-5 10.535350

0.103265025

1103223212 . 6

$310.3172,13510$

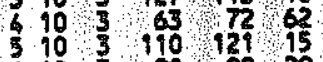

$610 \quad 3 \quad 90 \quad 9029$

$\begin{array}{rrr}-18 & 11 & 69 \\ -17 & 3 & 55\end{array}$

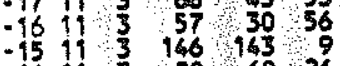

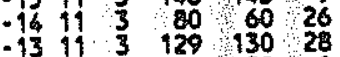

- 1211 3 01

$\begin{array}{lllll}-11 & 11 & 3 & 182 & 173\end{array}$

911,3 206 $190 \quad 15$

$-8113,344,40944$

$\begin{array}{llll}5 & 11 \\ -5 & 63 & 47 & 42\end{array}$

$4193,19 \quad 68919$

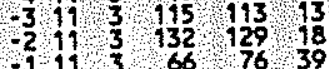

$1193 \quad 66 \quad 76 \quad 39$

$\begin{array}{lllll}011 & 3 & 147 & 149 & 13 \\ 119 & 3 & 159 & 197 & 10\end{array}$

$211, \frac{3}{232} 237$. 6

4113 3 9510413

$\begin{array}{llllll}5 & 11 & 3 & 3 & 0 & 33 \\ 18 & 12 & 3 & 0 & 17\end{array}$ h l 1080 10Fe 100

1012 3 101 \%1 11

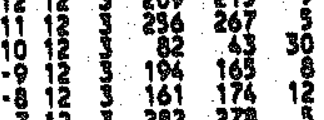

- 12 175 1710

113516,153

313100109

-19 13 3\% 916 है

- 16 15 $13 \quad 13516621$

- 143512612011

- 13520191913

.1013 3 61 औी 32

-6 $13 \quad 1651619$

- $-4 \quad 167 \%$

- 13 200 275

$013 \quad 053$

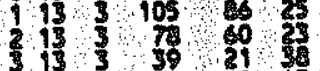

- 1414 150 130

$-13143,721018$

-11 I 165 148 10

$-91517176$

.14155150

-616 30 310 ?

- 14 10 83 20

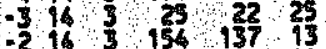

-116311 126 20

$17153 \% 40$ 28 45

$\begin{array}{rrr}16 & 5 & 3 \\ -15 & 15 & 302 \\ 105 & 20\end{array}$

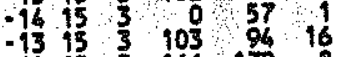

$-12153 \quad 166 \quad 1728$

- $1053 \quad 247266$

$\begin{array}{llllll}-9 & 15 & 3 & 171 & 159 & 9 \\ -8 & 15 & 3 & 173 & 155 & 7\end{array}$

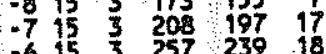

$\begin{array}{lllll}-6 & 15 & 3 & 257 & 239 \\ -5 & 18 & \end{array}$

$-415 \quad 3 \quad 91 \quad 9516$

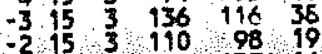

$-115,3161 \quad 13716$

$\begin{array}{lllll}1 & 15 & 3 & 85 & 74 \\ 7 & 83\end{array}$

$\begin{array}{ccccc}-16 & 16 & 3 & 72 & 100 \\ -15 & 95 & 82 & 18\end{array}$

$\begin{array}{rrrrr}14 & 16 & 3 & 987 & 118\end{array}$

$-12,16 \quad 3 \quad 122 \quad 127,12$

-1016 3 187 185 7

$\begin{array}{lllll}-9 & 16 & 3 & 158 & 152 \\ -8 & 16 & 13 & 53\end{array}$

\begin{tabular}{llllll}
8 & 16 & 3 & 3 & 53 \\
0 & 16 & 3 & 84 & 81 & 21 \\
-5 & 16 & 3 & 54 & 71 & 54 \\
\hline & & & 23 & 50
\end{tabular}
- 15 35\% 37

.111019

- $\rightarrow 15 \quad 22 \quad 201$

$114 \quad$ 3 11128 h I 10\% a 10Fe 10a

- 16 16 o -116 o 60 - 17 5 65 -1217 16 192 -10 17 40 20 40

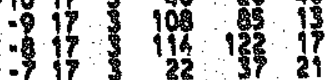
- 17 o 72 .3173006

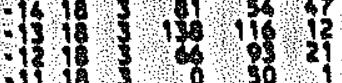
101010010715 - 163 130 116 2

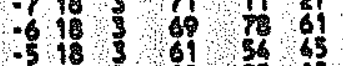
.121959625 $1119350 \% 40$ - $1903 \quad 0 \%$ ?

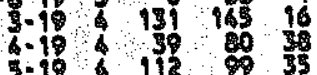
$3.19 \quad 1129035$

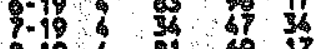
$8.19 \quad 81597$
$6.19 \quad 57$ $10-10 \quad 5752$

1069109 3.18 $7 \frac{75}{75}$ 1. $12 \% 19$ 618 o 1 7.18 00 ? 5 ? 9.19 59 58 50 $10-18 \% 110,92$ 12-18 का की 15 हो $-1-17$ 56 54 Q.17 117107 र $2-17602$ 3.17 160167 5.77661696 5. 17 6 52 6-17 185180 $8-17 \quad 57$ Z5 5 $9-7.416,2016$ $10-17 \%$ क 135 146 18 $12-17$. $164165 \quad 7$ $13-17 \quad 60 \quad 76 \quad 60$
$-3-16 \quad 65 \quad 77 \quad 24$ $-2-16 \quad 20 \quad 6619$ $0.16 \quad 46 \quad 43$ $0.16 \quad 92 \quad 8932$ $2.16 \quad 121 \quad 12313$ $3-16 \quad 176 \quad 144$ 5-16 265276 5116 16 178

$7-16 \quad 4 \quad 11187 \quad 16$ $816 \quad 4 \quad 977 \quad 18614$ $10-16$ 4 181 187 7

$\begin{array}{lllll}11-16 & 4 & 952 & 151 & 7\end{array}$ $13-16,4,106,107,15$ $14-16441$ ? 48,40 $-3-1544,0 \quad 851213$ $-2-15437 \quad 49 \quad 37$ $-1-15 \quad 4 \quad 137 \cup 115 \quad 12$ $0-154114,98,11$

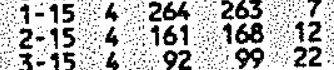

h. 11010 10Fe 103

3.1 15 । ? \%.15 10 is $0.13 \quad 101$

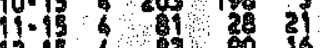
20-15 \& 13 121 $13-13$ - 13 . 129 - $=1.16 \quad 16,150$ -3.16 00 क - 14 IQ 158 is

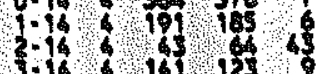
3.16 $161725 \%$ $5.16) 270203$ 7.1422315 9.164513950 10.164272266 $11-146123113$ I3. 4607 त 20 $16-14 \quad 4$ \& 65 \% 15-14 107 . - $6.13100 \%$

$-5.136092$ -4.15 140 136 22 -2.13 135 11622 0.13170 $1.13 \quad 101910$ $3-1360$ \% 5.13 \& 117 110 15 $6-13 \quad 116100$ $9-13 \quad 10^{\circ} \%{ }^{6} 12$ $10-13 \circ 77 \quad 82$ 11.1512693218 $12-1350910212$

$9693 \quad 472 \quad 60 \quad \frac{22}{75}$

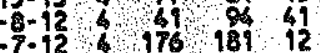

$6-92350130$

$-5-1261919$

$-6.124259238$

$-2-12 \quad 13511311$

9-12 17416910

$0-12 \quad 331314 \quad 6$ $\begin{array}{llll}1-12 & 4 & 8 & 41 \\ 2.12 & 27\end{array}$ $\begin{array}{llll}2-12 & 65 & 63 & 37 \\ 3-12 & 6 & 92 & 14\end{array}$

$6-12 \quad 466$ 29 23

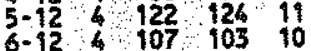
$7.12 \quad 669018$ $8.12 \quad 168137 \quad 7$ $10-12 \quad 310 \quad 336 \quad 8$

$11-12 \quad 4 \quad 92 \quad 8321$

$12-12 \quad 110 \quad 106 \quad 10$

$14-12491976 \quad 16$

$15-124248 \quad 74 \quad 48$

$-8-114 \times 107 \quad 86 \quad 20$

$-7114118,144$ 29

$-6-114452.7452$

$-4-114154,159$ 10

$\begin{array}{rlll}-3-11 & 4 & 274 & 287 \\ -2 & 18\end{array}$

$1-10409397$

$0-11,4$,44 422

$1-114200$ 207

$2-11,42442446$

3.11 4, 190 189 .

$4-114208227$ \& 


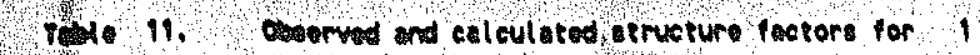

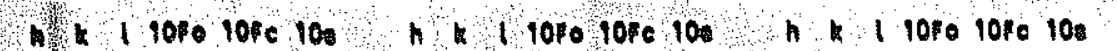

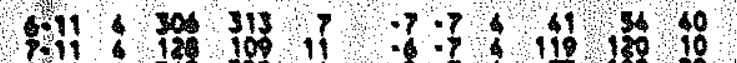

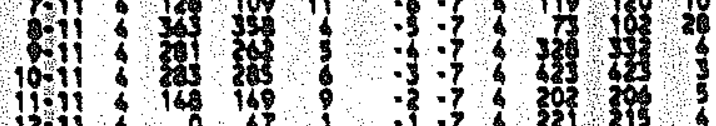
139190 181923

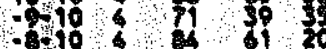

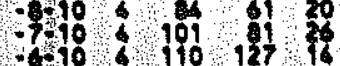
-479
$-670 \% 34263$

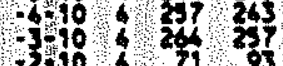
$+390 \% 71$ \% 19

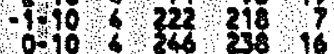
11106345245 3696279270 510 1914 $144^{9}$ 690
610 2431465

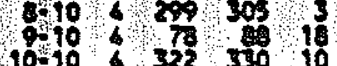
1010 \% 3223010 $1210 \%$ \% 1914 1410 का 174 \%

-10-9 10 133 12 -9 153 162 12 $\begin{array}{ll}-8 & 0 \\ -7 & 5\end{array} 5350$ $-6-9$
$-9-9$ 1731679 -5-9 179167

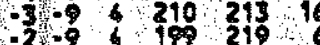

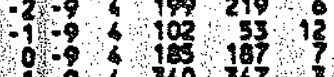

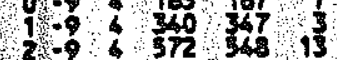
5-9 2227 4. 15 13 6. 31735 7.9211 20 $9-9.167162$

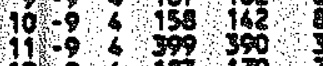
11.96192170 12.9 176 173 9 $16-9$ - 76 - 78

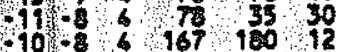

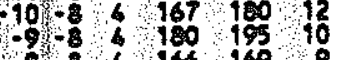

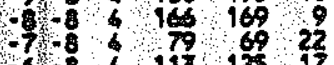
-6 19312517

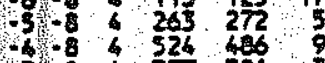

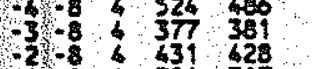
-94 6321317

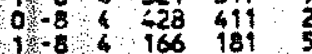
1)-8. 561526

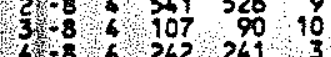

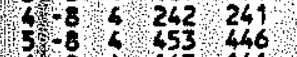
$61-84143149$ $8=8 \% \frac{452}{350}+$ $\begin{array}{lllll}90 & 0 & 233 & 236 & 4\end{array}$ $11-8,4$ 52 $58 \quad 34$ (3)

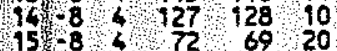
$1276 \quad 61 \quad 6260$ $-10-7$ का 286 284 11 $\begin{array}{rlll}-96-7 & 164 & 137 & 9 \\ -8.7 & 249 & 246 & 9\end{array}$

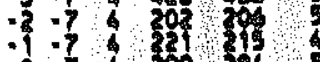

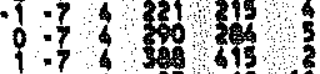

-7 .
-7 की

7
$3=7$

5.7
-7

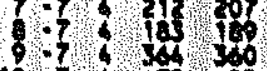

10.744 का 139

$13-7,619 \% 19$

$4.76 \%$ का

$19.6 \% 9620$

116610012810

$.9 .6 \% 320312$

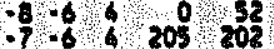

$\begin{array}{ccc}-7.6 & 6 \\ -6.6 & 205 & 209\end{array}$

- -6 11017

- $-6.200 \%$

- . 10135

- . 37 का

$1-6 \% 007$

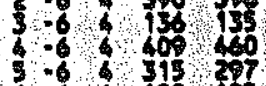

8.6 .1901

$\begin{array}{lll}7 & -6 & -67\end{array}$

10.648 \% 78

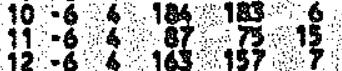

$-6100101$

$13-5109012019$

- 12 -5 177105

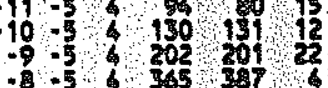

$\begin{array}{lll}-7 & -5 \\ -6 & -5\end{array} 39353$

$\begin{array}{lll}-6 & -5 & 353 \\ -5 & 543\end{array}$

- -5.5600195919

$-3-54326459$

- $-5 \quad 6 \quad 426459$

0.540379216

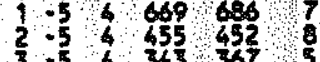

$3-5 \quad 345$ 367 35

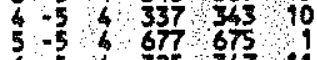

$\begin{array}{lllll}6 & -5 & 4 & 325 & 37\end{array}$

$8-5 \quad 16015511$

$9-54042-1 \quad-15-14$ o5 9420

$10-5$ \% 160 135 8 - $14-1$ \& 121 122 16

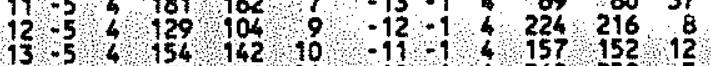

$13.5,4,75, \ldots 62,21$

$-14-4,490125,40$

$-13-64 \% 96,91,22$

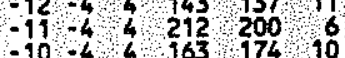

$-10-4 \quad 4 \quad 163$ 174 10

$=0-4,4,68$ और 11235 -7.44235 232 8

$-6.4144330 / 293$.

$-4-4,4$ 491 522 अ 14

$-3-44660643,6$

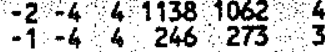

$7.4 \quad 4$ 4

- 422219

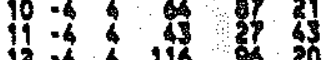

$12-64.116$. 20

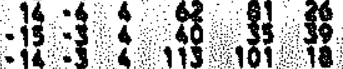

$-13-34613119$

$-11.31013$

$\begin{array}{lll}-9 & -5 \\ -8 & -3 & 1\end{array}$

- -361960

- -3.34 45 45

3. 5131

7 12 I5

90.3 \& 147 ?

11.314163

13 . 61

$-15-2,46106$

$-13-2 \quad \begin{array}{lll}76 & 13 \\ -1 & -2 & 35\end{array}$

$-11.262131010$

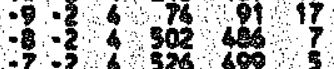

7.265660

$5-403920$

$-3.24167175$

$-1 \quad 2 \quad 68060$

0.2638450

3.21516582 ?

$5.2,35555114$

6.2
$7-2$ 665456

$\begin{array}{llll}8-2 & 143 & 139\end{array}$

-10.14260258 . 5

$-9.14285,288,10$

$-7-1$ द 633 625 द

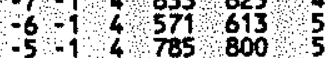

$-4.14,169,175,6$

$-3-1412191257$ ?

$0,-14$ 4 232 , 230 , 1

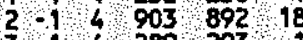

$\begin{array}{llllll}5 & -1 & 4 & 685 & 701 & 14 \\ 6 & -1 & 4 & 323 & 326 & 8\end{array}$

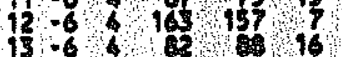

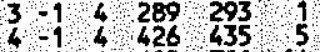

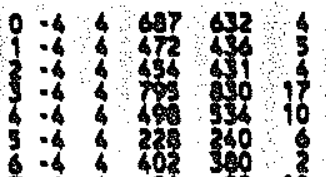

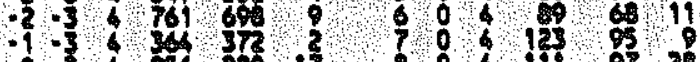

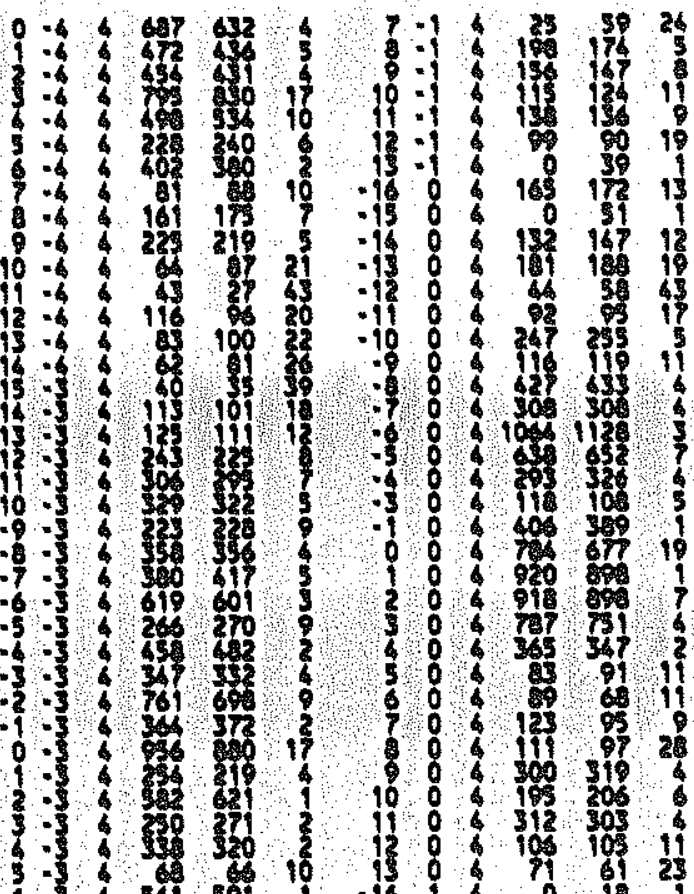

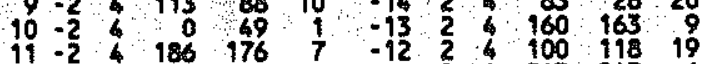

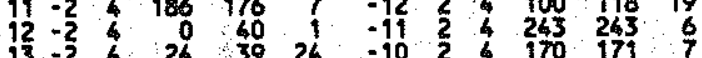

$\begin{array}{llllll}-5 & 4 & 309 & 328 & 3 \\ -5 & 2 & 874 & 858 & 2\end{array}$

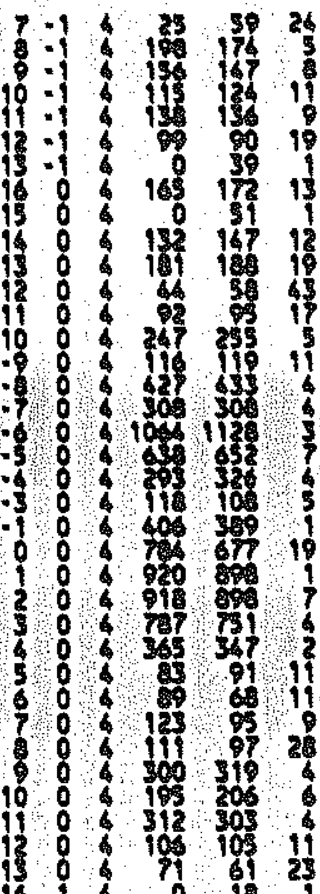

$-161901901$

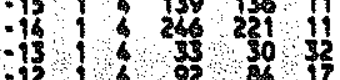

-11 220203

- 21235

$-7.64628$

- 15012310

- 15150

- 1 \% 3745

21 103165

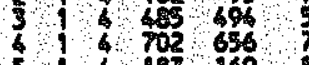

$51 \quad 2351698$

81201515

$101 \quad 222 \quad 206 \quad 8$

$\left.11 \quad l^{16}\right)^{9}, 10$

$1254 \quad 710826$

$-16240022$

$-152 \quad 002820$

$\begin{array}{rlll}-10 & 2 & 170 & 179\end{array}$

$\begin{array}{llllll}-8 & 2 & 4 & 28 & 129 & 131\end{array}$

$\begin{array}{lllll}4 & 2 & 4 & 1495 & 1502 \\ 3 & 2 & 417 & 400 \\ 328 & 330\end{array}$

-124354332 ?

1) 2 4 351 , 359 र 8

224410376,8

$32{ }_{4} \quad 396 \quad 406 \quad 18$

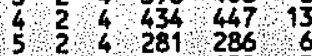

6224479452 , 2

824228 239. 6

$924121,122 \quad 10$

$11 \quad 2 \quad 4 \quad 128 \quad 128$ h I lopoloro 100

:16 $16 \quad 0110$ 15

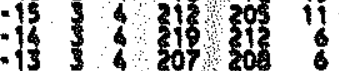

-12 190

$-10 \quad 3 \quad 03$ is

- 54541

- $\quad 059$

- 340142

- 40740

- 445310

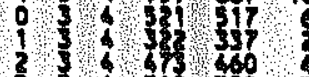

$354319,53 \quad 3$

5. 400 311

73568356

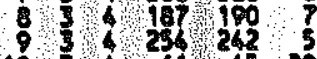

$1036 \% 25$

.1756076246

1511516131

$14 \quad 13514017$

$12 \quad 109190$

$-11 \% 167165$

$\begin{array}{lll}-10 & 1280 \\ -9 & 6000\end{array}$

- $\quad 216212$

$-646020$

- 461655

-3 421219

-1 s5 57

1459622

26611360 ?

\% 150129

$3410 \%$ ?

$7 \% 131$ 132 ?

$86 \quad 20919025$

1016496

-185456 o 54

$16 \quad 6160160$

$-155412312416$

$\begin{array}{llll}-14 & 5 & 170 & 177 \\ -13 & 11\end{array}$

125183188

$\begin{array}{llllll}-10 & 5 & 4 & 191 & 206 & 8\end{array}$

$\begin{array}{llll}-9 & 5 & 4 & 117 \\ -8 & 416 & 13\end{array}$

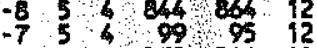

-6 $5 \quad 575 \quad 5949$

- $5 \quad 5 \quad 81077023$

-3 $5 \quad 4 \quad 140$ 158

-2 54691 675

-1 $5 \quad 435446$

$154 \quad 4494192$

$25484 \quad 8537$

4 5 $4 \quad 365 \quad 347$

5 5 4 181 189 10

644157 . 162 ?

$8 \% 4107,103,15$

$954463 \quad 62$ 33

$10,5,4,77 \quad 22$ 23

$-17.64469,15422$

$15 \% 4 \quad 50,7649$

$1464,98 \quad 6120$

1364,86 66 38

116434260 


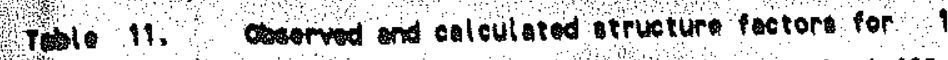

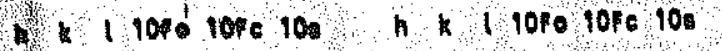
(1) $\begin{array}{lll}19 & 179 \\ 0 & 16\end{array}$ - 1320 -3. 30 -1. 100

13190

(1.615 140

16.62527

(1. 105 . 13611 $98 \% 1690$ $-17716143$ $437 \quad 276379$ $-167220159$ 1276z 271 -1072215 218 476606 $797 \quad 35951$ +6 7200 47.63519 - $7 \quad 350$ 1 7 227 230 $\begin{array}{lll}0 & 7 & 232 \\ 200\end{array}$

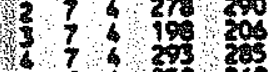

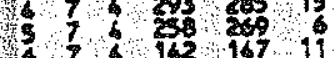
7.74721002 15 100 10 19 17 . 6576 13 \& $110 \%$ 375 की 115 12 क 167 , 169 $10 \% \frac{253}{216}$ +78 206 319 6. 632332619 $\begin{array}{lll}0.5 \% & 176 & 175 \\ -3 & 539 & \end{array}$ -3 5316286 -2 $17 \%$

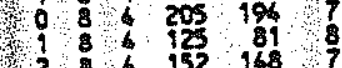
$\begin{array}{llll}2 & 152 & 168 & 7 \\ 3 & 92 & 13\end{array}$ 68 117 11512 6 $8206205 \mathrm{~g} 2$ 18.123 .7559

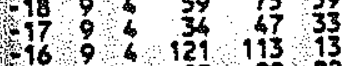

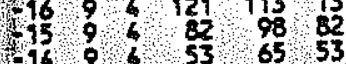
$16996223165 \%$ $152,4 \quad 115$. 139,13 11094263,249 ? 8996411960815 $7 ? \div 210 \quad 12419$ $69,6291,273$ - 399,4222 220 4 $-2 \% ? 141$ o3 11

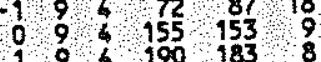
29.190183 .86
3. 117110012 - 192605 -1910 $10 \% 65$ -1 $10 \% \quad 100 \quad 100,10$ - $15 \% 10710310$ $1210 \quad 1313710$ $610 \% 3 \%$ का

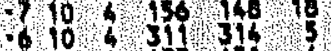
3106172026 $-3106736930$ 610.2281916. 010615613111 3100 117 117 19 61062011937 3. 101013016 $-19105 \%$ $-1911001013$ - 1114176 160 -11
-13
-12 .11114230269 - 11 o $10 \%$ $\div 11 \quad 306199 \% 11$ -6 11. 45523519 $-611421520978$ - 19 190 969 - $11 \% 12713710$ 0116211202 2110679 का 3 $11.171912,15$ $511 \% 6913$
-181260 $-1712 \quad 216210$ - $1512+17819211$ - 1132301309 $\begin{array}{lll}-13 & 12 & 129 \\ -12 & 12 & 139\end{array}$ -11 2 क 180 166 19 - 12 . 199189 $-712 \quad 4 \quad 145 \quad 118,16$ $-7126183163$

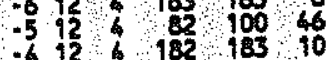
- $1212 \quad 1821858$ - $12 \quad 4 \quad 8810140$ $-112 \quad 1631938$ $11^{2} \quad 42 \quad 6342$ $312 \quad 194 \quad 195 \quad 7$ -18134 , 75,7431 17134469 . 949 $-16134235,245$. 6 $16134218,195,9$

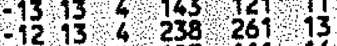
$-1134,123,111,16$

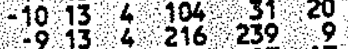
$8134119 \quad 97 \quad 17$ $-7134128 \quad 114,13$ $\begin{array}{llllll}-6 & 13 & 4 & 123 & 108 \\ -5 & 133 & 13\end{array}$ $\begin{array}{llllll}-4 & 13 & 4 & 322 & 332 & 9 \\ -3 & 13 & 4 & 83 & 100 & 27 \\ -2 & 13 & 4 & 67 & 73 & 29\end{array}$ $1010 \% 607397$ h 1 10Fo 10\% 10

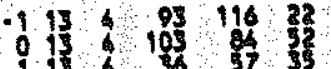

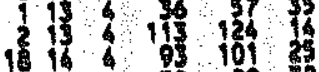
$\begin{array}{rlll}-17 & 16 & 5 & 30 \\ 9 & 35\end{array}$ -15 is $137 \quad 14075$ -13 I6 128207 I -11 140130 - $1010.72{ }^{23}$ -716 16 1 3146176173 ?

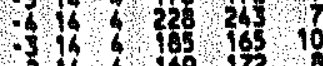

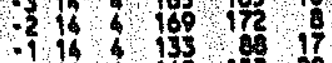
0161431520 $\begin{array}{rrr}171 & 63 & 25\end{array}$ -1615105 का 16 $-161513725$ $-12 \quad 6 \quad 27395$ $-1014231$ - 15220216 -715 117 20 - 6151173930 - $15 \quad 16112150$ - $15 \% 53$ $015 \% 71 \%$ $1616 \quad 100,1322^{9}$ -1616 I $^{16} 12$ $-13164190130$ .111616275230 $-916 \quad 271520$

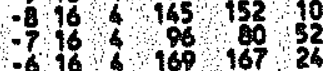
$\begin{array}{lll}-6 & 16 & 169 \\ -5 & 167 & 2 \\ - & 0\end{array}$ - $16,427 \quad 5227$ $\begin{array}{rrrrr}-2 & 6 & 63 & 49 & 45 \\ -13 & 77 & 0 & 26 & 1\end{array}$ - 13176 o $^{0} 1$ $\begin{array}{llll}-15 & 7 & 47 \quad 26 \quad 46\end{array}$ $-11 \quad 17 \quad 216 \quad 200 \quad 5$ $-917 \quad \begin{array}{lll}89 & 127 \quad 16\end{array}$ $-7174 \quad 2512519$ $-61746 \quad 1913$ $\begin{array}{lllll}-5 & 17 \quad & 0 & 37 & 1 \\ -4 & 17 & 70 & 41\end{array}$ $-1418445 \quad 8145$

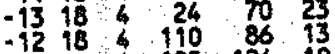
$-11.18 \quad 12512410$ $\begin{array}{rrrrr}-10 & 8 & 69 & 54 & 54 \\ -10 & 18 & 105 & 106 & 54\end{array}$

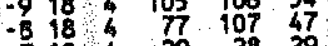
$\begin{array}{llll}-718 & 29 & 28 & 29\end{array}$

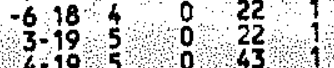
$\begin{array}{lllllll}4-19 & 5 & 0 & 43 & 1\end{array}$ $6.995,34 \quad 6834$ 7-19 5 0,70 , $\begin{array}{lll}8-995 & 83 & 68 \\ 9 & 19 & 18\end{array}$ 10-195 $0 \quad 32$. $\begin{array}{lll}1.18 & 5 \\ 2.18 & 5 & 23\end{array} 51,23$ $\begin{array}{llll}2-18 & 5 & 5 & 01 \\ 3-18 & 105 & 20\end{array}$ $4-18,5,30,39,30$ $6.18541 \times 30 \quad 40$ $7.185,113,92,13$ h K / 10Po 10\%0 10 $\begin{array}{lll}0.19 & 5 & 5 \\ 10.10 & 54\end{array}$ 11.18 140 $-1.17 \quad 151 \quad 150 \quad 16$ 1.17 56 \% 3.17 514912014 4.1720 \%. 50 o 25 .17 1451610 $90.97 \quad 5 \quad 675$ 1217517919 $\begin{aligned} & 13.17 \\ & -3.16\end{aligned} 102,132,10$ $1.16,146$ a 15 1.16511115 18 3.165051 - 165176 176 10 $6.16 \quad 5 \quad 303 \quad 302 \quad 12$ $7 \% 56 \quad 167 \quad 13351$ 8.165 क 5857 10.16 5 10 115 11 $11-165 \frac{5}{7} \quad \frac{96}{5} 17$ $\begin{array}{lll}13.16 & 0 & 31 \\ -4.15 & 5 & 22\end{array}$ $\begin{array}{lll}-3.15 & 62 & 45\end{array}$ - $-15 \quad 105 \quad 78$ 0.15
1.15 $2-15549$ $3-15 \quad 2632372$ 3-15 5126135 6.15 5 109 264 12 8.155183165 $10-15 \%$ का 76 $11.15,516017127$ $12-955120175$ ? 6-15.5 1610111$)^{9}$ - -1653920119012 - 355 5 1613 $\begin{array}{lll}-2-14 & 5 \\ -1-1 & 5 & 9\end{array} 11911$ $0-165216209 \quad 5$

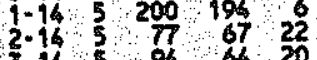
$3-1459420$ $4-145 \quad 176 \quad 174 \quad 8$ $\begin{array}{llll}5.14 & 5 & 132 & 128 \\ 6-14 & 5 & 98 & 9\end{array}$ $\begin{array}{lll}6.16 & 5 & 107 \quad 108 \quad 15\end{array}$ $8-14 \quad 38 \quad 29 \quad 38$ $10.94 \quad 0 \quad 24$ $11-14 \quad 5 \quad 1221111$ $12-14 \quad 5 \quad 116 \quad 116 \quad 12$

$14-145 \quad 15$ b4 14 $\begin{array}{lll}-7.13 & 5 & 99\end{array} \quad 84 \quad 17$ $\begin{array}{llll}-6-13 & 5 & 122 & 15 \\ -5-13 & 5 & 0\end{array}$ $\begin{array}{cccc}-4-13 & 5 & 82 & 86\end{array}$

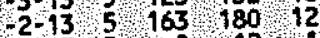
$-1-135,0$, 12 $\begin{array}{lllll}0-13 & 5 & 190 & 185 & 7\end{array}$ $2-135198192$

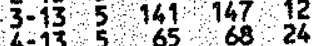
4-13 $5, \quad 65$ प $101 \quad 17$ $6-135,50,42 \quad 28$ $8-13$ 5 $315,301,3$ $9-135110 \quad 130,14$ h l lopo 10Pe 108 131314 का 15 1 की -7. 110 -6.12 Is 135 $-5-12 \quad 5 \quad 256$ 261 8 $\begin{array}{rl}-3.12 & 578273\end{array}$ $1.12 \quad 2302017$
0.1250

.1234625 $3-1251151018$ $4-125196100$ $5.12,52629$ 9.12523025 8.125 35 350 $10.125,93190$

11.12519 रक 21

$13-1251601426$

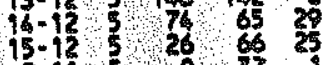
$-9.1150037$ $.8-115158,4758$ - $6-115155 \quad 33 \quad 50$ - -111500325 -2-19 5246 221 8 0.152052005 $1-115275$
$2-11502$ $3-195181$ 187 10 5-11 5380 is 10 6-115 511 26

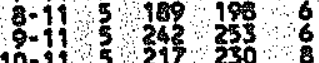

$11-11$ क 10 क 10715

$\begin{array}{llll}12-11 & 5 & 181 & 174 \\ & 6\end{array}$

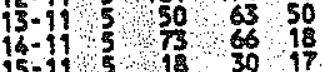

$15-115,18$. 10 ?

$-9.905152 \quad 4252$

$-7-105 \frac{5}{5} 512$

$-6-10$ 5 61 49 36

$\begin{array}{lll}-5-10 & 5 & 0 \\ -56 & 76 & 11\end{array}$

$-3-105 \% 9616$

-2-10 5 154 16011 $-9.10 \quad 5149$ 149 8 $1-905$ । 013 2-10 $5216 \quad 2340$ 5190 $5.10 \quad 5301 \quad 269 \quad 10$ $6-10 \quad 5 \quad 204 \quad 210 \quad 6$ 7.105229235 $9-10 \quad 5 \quad 129138$ $\begin{array}{lllll}10-10 & 5 & 313 & 335 & 8 \\ 11-10 & 5 & 323 & 321 & 6\end{array}$ $\begin{array}{cccc}11-10 & 5 & 325 & 321 \\ 12-10 & 82 & 20\end{array}$ $\begin{array}{lllll}13-10 & 5 & 68 & 31 & 19\end{array}$

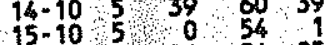
$-11.95,26,54,25$ $0-9568$ 58 29 $\begin{array}{rllll}8.9 & 5 & 65 & 108 & 64\end{array}$ $6-95290275 \% 6$ $-5-95187$ 159 8 $\begin{array}{llll}-3 & -9 & 5 & 205 \\ 212 & 12\end{array}$ $295333 \quad 358$ 
7.t

Pap 10

Telo 11. coerved al eulated etructure fector for

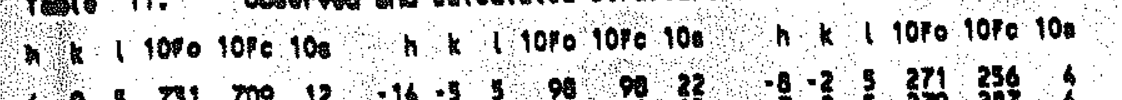

19 5 7210012

1. 350

\% 1000

$10 \%$ 10 100

$121 \quad 76 \quad 09$

15 10 01

13 31 क

$1196 \%$ \% 90

$-98522826312$

7.85 70,7326

+5 160 171 11

- 10 , 2092106

$-3-8,509610$

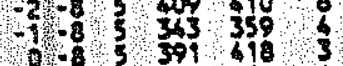

9.8. 67565

3.8 5 375 367

5.8 316920

6.: $5200 \%$

7. 31519

1. $519710 \%$

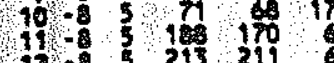

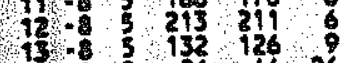

$14-8$ 5 53 क 52

$-12-753$, 30

$\begin{array}{lll}-10.7 & 5 & 192 \\ -9 & -7 & \end{array}$

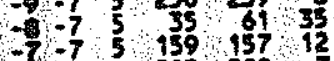

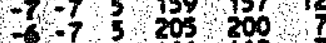

- -7 5 1112

$\begin{array}{lll}-4-7 & 569\end{array}$

$-2-75$ क 117 , 10819

$9-7$ 5 200 217

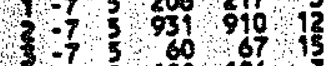

$\begin{array}{llll}5-7 & -7 & 180 & 186,3 \\ -7 & 5 & 403 & 428\end{array}$

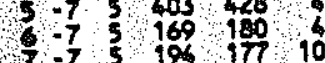

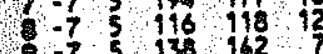

$\begin{array}{lll}5 & -7 & 138 \\ 73 & 7\end{array}$

$\begin{array}{lll}19-7 & 5 & 73 \\ 11-7 & 5 & 76\end{array}$

$\begin{array}{llll}12 & -7 & 5 & 148 \\ 3 & -7 & 02 & 59\end{array}$

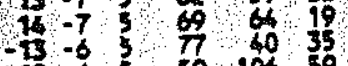

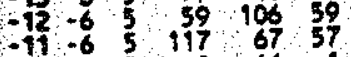

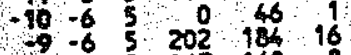

$\begin{array}{llllll}90 & -6 & 5 & 202 & 180 & 10\end{array}$

\begin{tabular}{lllll}
\hline 0 & 5 & 139 & 132 & 18 \\
\hline 0 & 5 & 201 & 183 & 16
\end{tabular}

$\begin{array}{llllll}5 & -6 & 5 & 201 & 183 & 16\end{array}$

$\begin{array}{llllll}6 & 6 & 567 & 295 & 47\end{array}$

$\begin{array}{ccccc}-3 & -6 & 5 & 55 \\ 2 & -6 & 5 & 417\end{array}$

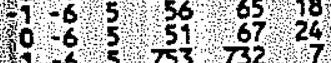

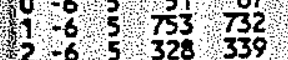

$2.6,5,200,205$

5 -6 5383.408

$\begin{array}{llll}6 & 6 & 5 & 41 \\ 3 & 39 & 28\end{array}$

$1665,301,309,26$

$806 \quad 2572253$

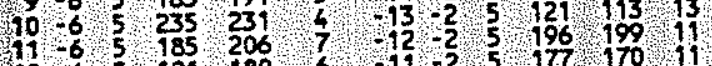

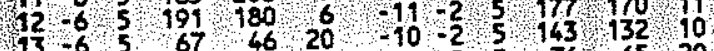

$\begin{array}{lllllllllll}13 & -6 & 5 & 67 & 46 & 20 & -9 & -2 & 5 & 76\end{array} \quad 65 \quad 20$

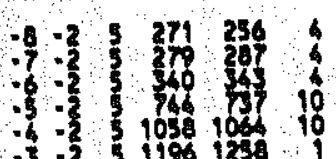

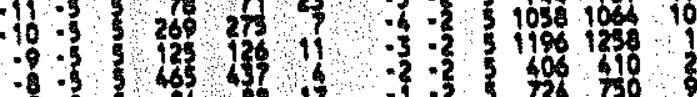

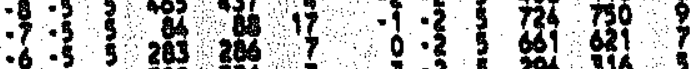

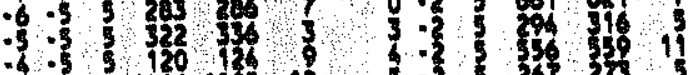

-3. 1014105

$0.5,51 \%$

2.5.5. 361 , 53

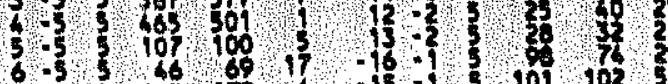

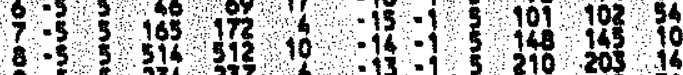

10.5. $5233,237,26$

$12-5,1296$

$13.5,56,53$

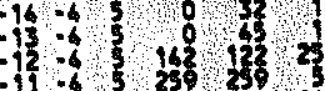

$-11.6325070 \% 1$

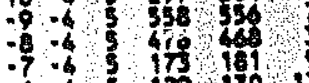

$-6-6350 \% 5 \%$

$\begin{aligned} & -2 \\ & -1\end{aligned}-65434069$

$0.65616378,7$

$3=4548675$

4.656460

$\begin{aligned} & 5.6 \\ & -4\end{aligned} 281,270$

8.6546710

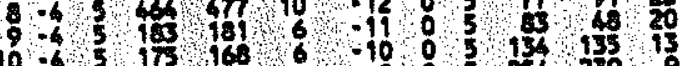

$11.6,5,46727$

12.65162138910

$14-6 \quad 5 \quad 5620,32$

$-16.3577 \% 1912$

$-12=350900$

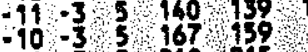

$-9.35210 \% 15$

$\begin{array}{lll}-6 & -3 & 5 \\ -5 & -3 & 271 \\ -3 & 281\end{array}$

$\begin{array}{llll}-4 & -3 & 5 & 222 \\ -3 & -3 & 5 & 925\end{array}$

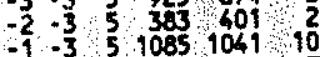

0.35541563 . 5

$\begin{array}{lllll}2 & 3 & 5 & 353 & 328\end{array}$

$\begin{array}{llll}4 & 3 & 5 & 319 \\ 5 & 5 & 280 \\ 3\end{array}$

$6,35,276 \times 272,10$

$\begin{array}{lllll}8 & 3 & 5 & 62 & 62 \\ 9 & 52 & 200 & 198\end{array}$

$\begin{array}{ll}10 & 3 \\ 11 & 3\end{array}, 86, \quad 95,150$

$12-3,5,72,90018$

$\begin{array}{lllll}13 & 3 & 5 & 105 & 205 \\ 5\end{array}$

$-14-2,5183$. 183,10

$13=15210 \quad 20316$

$.10 .1 \% 35,36$

$-9.1 \quad 33939$

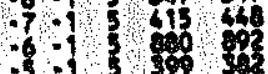

-1 \%os 102

$\begin{array}{lll}-2 & 191 \\ -1 & 0\end{array}$

$3-1 \quad 26320$

5. 5212 210

6. 154113

$8-1514140$ 15

$12-1$ ) 27

$-160353515$

- 15 o 5164510

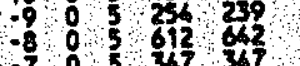

-6 053156

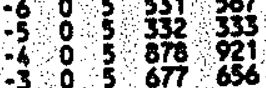

$\begin{array}{llr}-2 & 0 & 64027 \\ - & 0 & 050\end{array}$

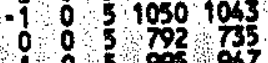

2. 0,59597

$405 \% 10626$

$\begin{array}{llll}5 & 0 & 346319 & 6 \\ 6 & 0 & 519 & 328\end{array}$

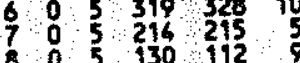

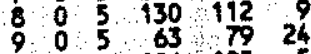

$\begin{array}{llllll}10 & 0 & 5 & 196 & 185 & 5 \\ 11 & 0 & 5 & 29 & 63 & 28\end{array}$

$\begin{array}{llllll}11 & 0 & 5 & 29 & 63 & 28 \\ 12 & 3 & 5 & 56 & 67 & 54\end{array}$

$\begin{array}{cccc}-17 & 5 & 0 & 17 \\ -16 & 5 & 176 & 160\end{array}$

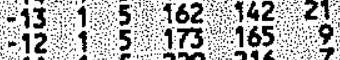

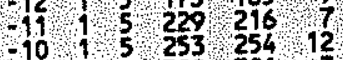

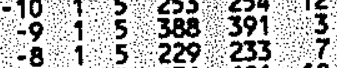

$-81,5631$ 601 10

$61,5262,294,4$

-5 1) 5 $704 \quad 409$, 2

$\begin{array}{llllll}3 & 1 & 5 & 770 & 806 & 20\end{array}$

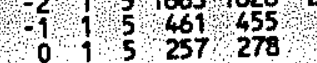

$\begin{array}{lll}-6 & -6 & 5 \\ -5 & -4 & 20\end{array} 6$

-7 - 352552465

$10=13 \% 3$

$-15,35,30,50,30$

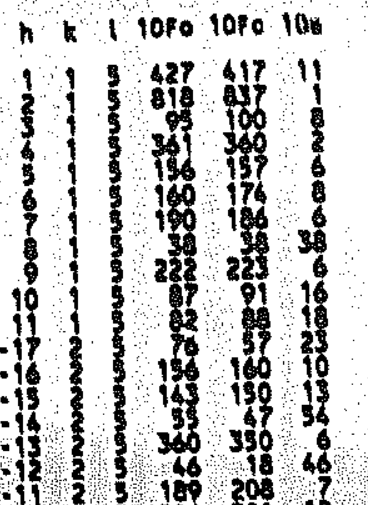

h lo lofa lofe 100

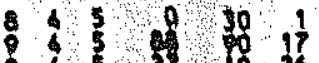

$\begin{array}{rr}10 & 5 \\ .18 & 5\end{array}$

16 I2 III I

is 170028

.121017

-10 100 13

-1 s23\%?

$.65 \% 46796$

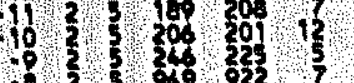

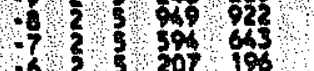

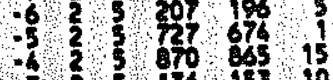

035136153

-12527 $2 \pi n$

123215176

3. 3 27 276?

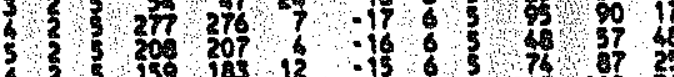

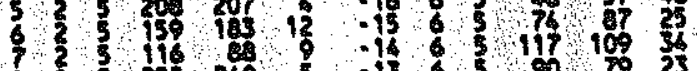

$82525526052130 \%$

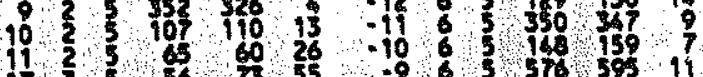

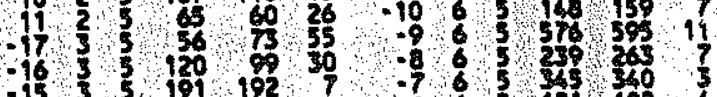

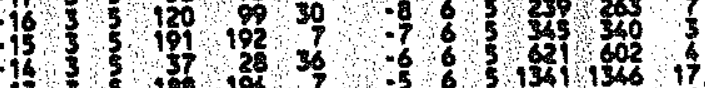

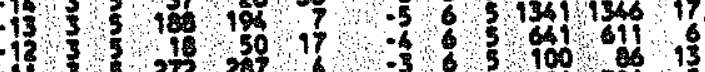

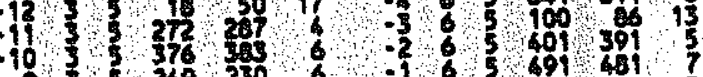

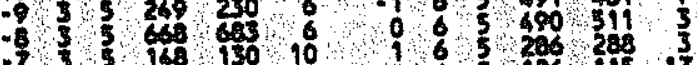

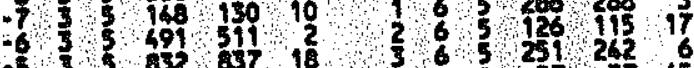

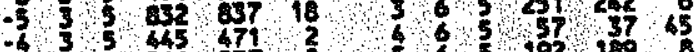

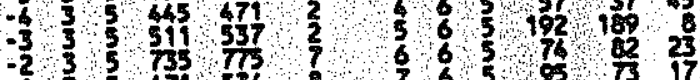

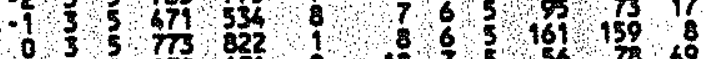

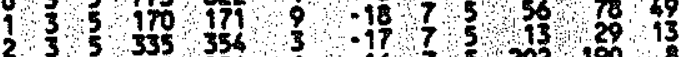

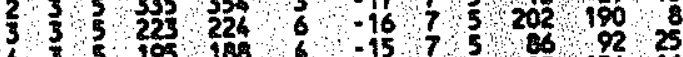

$43519518560-15751651516$

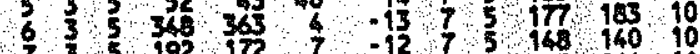

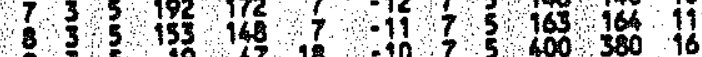

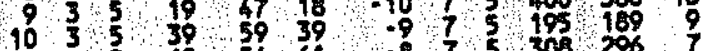

$-18 \quad 562561$

$-176587839$

$\begin{array}{llll}-16 & 4 & 5 & 107 \\ -15 & 54 & 10\end{array}$

$\begin{array}{lllll}-14 & 4 & 5 & 213 & 204 \\ -13 & 5 & 182 & 178 & 10\end{array}$

$-12 \quad 5 \quad 93 \quad 64 \quad 16$

$\begin{array}{llllll}-11 & 5 & 312 & 306 & 4 \\ -10 & 5 & 205 & 208 & 6\end{array}$

$\begin{array}{lllll}-10 & 5 & 505 & 208 & 6 \\ -9 & 5 & 97 & 121\end{array}$

7. 4, $5185,381, \quad 3$

$465,580,590,14$

$445,708705.5$

$345,571,588,10$

$24,5,803,797$

$1+4369396$

145202,209

$\begin{array}{lllll}3 & 4 & 5 & 150 & 144 \\ 4 & 5 & 242 & 242 & 5\end{array}$

- $753082 \%$

$\begin{array}{ll}-7 & 75100 \quad 320\end{array}$

$\begin{array}{lll}-5 & 7 & 5036358\end{array}$

$\begin{array}{lllll}-4 & 7 & 5 & 364 & 338 \\ -3 & 7 & 5 & 218 & 235\end{array}$

$-2 \quad 7 \quad 27425311$

$\begin{array}{llll}0 & 7 & 5 & 249 \\ 0 & 257 & 13\end{array}$

$175 \quad 44 \quad 67 \quad 43$

375,19319016

5.7.5.185. 199

$67,5,109,107,15$

$\begin{array}{ccccc}8 & 7 & 5 & 0 & 9 \\ 18 & 8 & 45 & 5\end{array}$

-168 . 5 253 262 \%

15.85 $2000116 \quad 11$

3385124,11418

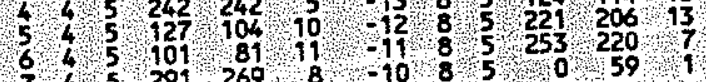


H.

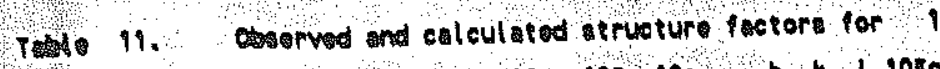

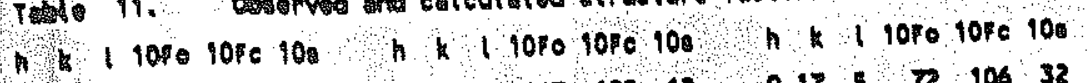

-9) 195194

- .

190

\% 190

- 5 35 35

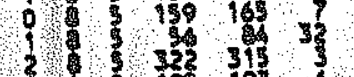

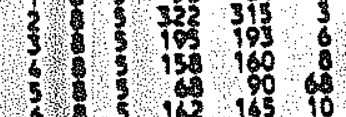

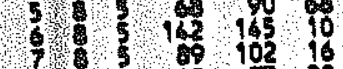

$-1795 \%$ o 13

-16 9 क 1919

41695280 zas

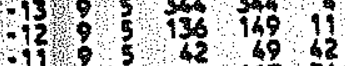

$-11095126117$

$9 ? \frac{59}{396} 160$

-7. 16710010

$\begin{array}{rr}6 & 57 \\ -3 & 310\end{array}$

3. 2100

$\begin{array}{ccc}-2 & 165 & 122 \\ 0 & 253 & 235\end{array}$

130511813

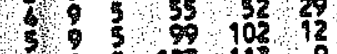

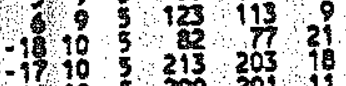

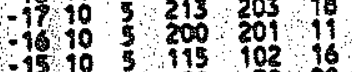

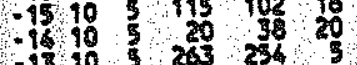

$\begin{array}{lll}-1310 & 253 & 234 \\ +1210 & 326\end{array}$

$1110 \quad 127$ as 16

$\begin{array}{rlll}-9 & 5 & 5 \\ -10 & 50\end{array}$

$-710 \quad 5 \quad 651095$

$-610 \quad 5$ 2 15 20 235

- $10 \quad 5 \quad 327 \quad 316 \quad 11$

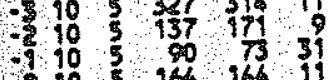

$910 \quad 5$ 166 14719

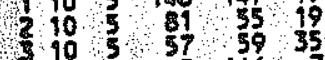

$4105165 \quad 146 \quad 7$

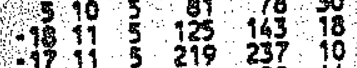

1619 5062

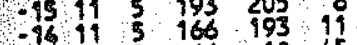

$-1311 \quad 601045$
0

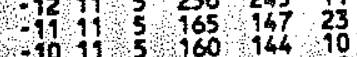

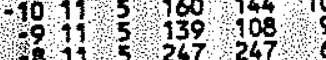

$78115,267.247 .49$

$611, \frac{80}{5}, \frac{62}{12} 14$

G 115,104 113 15

13 $119 \begin{array}{llll}543 & 258 & 6 \\ 5 & 116 & 133 & 13\end{array}$

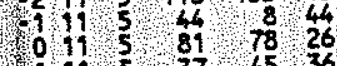

19153745 36

$211 \% 5911113 \%$

$4812,5,80,53 \% 18$

$\begin{array}{llllll}18 & 12 & 5 & 86 & 106 & 18 \\ 717 & 12 & 5 & 168 & 130 & 9 \\ 15 & 12 & 5 & 120 & 121 & 14\end{array}$

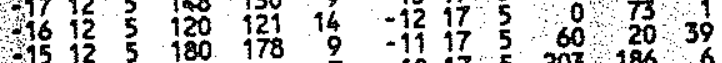

$\begin{array}{rrrrrrrrrrr}115 & 12 & 5 & 180 & 178 & 9 & -11 & 17 & 5 & 600 & 200\end{array}$

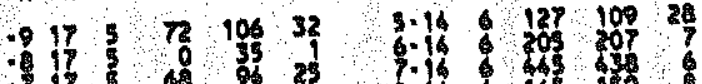

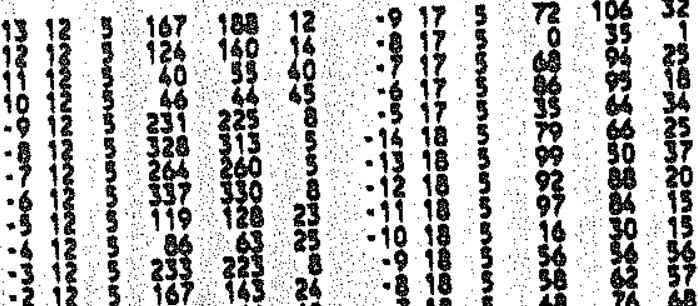

$.712,167,1636$

(1) $5129,120,18$

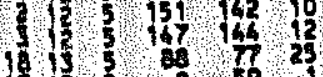

$-1915,50,5969$

$-1613,519220078$

$12135170185,8,3-186468$ s5 68

10131159923

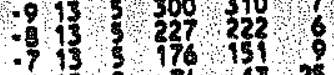

130.186205 208 11

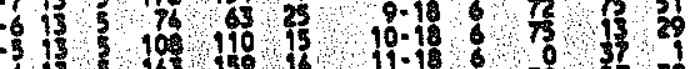

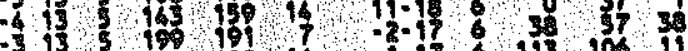

- 1350512016

1 13 का 131 ?

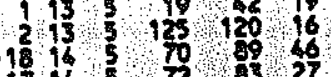

$-1765928327$

- 15316919310

$-16163216 \quad 227 \% 22$

$12165222 \quad 220$

- 10 16 250721910

- 163272

-716 3 217 213 6

$-6143147161$

-3145 17? 169 17

- 115188978

-17153 5 66 22 66

- 15 15 15 203 200 क 17

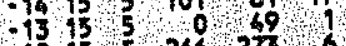

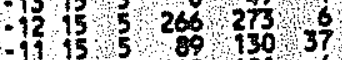

- 10 15 5 $605 \quad 60146$

$\begin{array}{lllll}-8 & 5 & 5 & 66 & 71 \\ -7 & 5 & 0 & 68\end{array}$

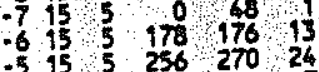

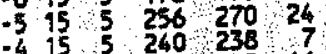

$\begin{array}{lllll}-3 & 5 & 5 & 45 & 56 \\ -2 & 5 & 53 & 53\end{array}$

$\begin{array}{llll}-1 & 55 & 5 & 20 \\ & & 5 & 39\end{array}$

$\begin{array}{rllll}15 & 16 & 5 \\ -14 & 6 & 0 & 93 & 1 \\ 92 & 93 & 25\end{array}$

$\begin{array}{llll}13 & 16 & 5 \\ 2 & 144 & 137 & 140\end{array}$

$11016,5,130,130,11$

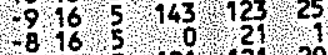

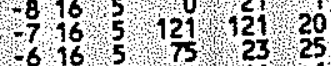

$\begin{array}{lllll}6 & 16 & 5 & 5 & 23 \\ -5 & 16 & 25\end{array}$

416 5 149 , 14014

$\begin{array}{llllll}-316 & 5 & 123 & 114 & 17\end{array}$

$-1.17 \quad 61310011$

0.17 or 100

$2.7 \quad 610$ iss 18

6.17.6 of 19317

6.17670112

7. 17.6108 104 12

$8-176$ 135 136 i1

10.17 6 क 327

12.17606053

- $-16 \quad 6791216$

$-2.16 \quad 6 \quad \frac{77}{75} 104$ ?

$0-1663^{0} 31$

2-16, 612212115

$\begin{array}{llll}3.16 & 6 & 113 & 124 \\ 4 & 16 & 13\end{array}$

5.16 . 18 क

$6.16 .6{ }^{14} 11826$

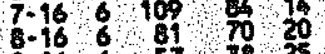

$9.16 \quad 6 \quad 57 \quad 3625$

$10-166$ \% \& 12

$11-16 \quad 6$ ? 13012

$12-160612912111$

$\begin{array}{lll}-5.15 & 6100 \\ -6.15 & 61 & 89\end{array}$

-3. $156162100 \quad 16$

$\begin{array}{rrr}-2.15 & 6 & 96\end{array}$

0.15610010912

$\begin{array}{llll}1-15 & 6 & 205 & 218\end{array}$

$\begin{array}{llll}2-15 & 6 & 67 & 71 \\ 3-15 & 6 & 15 & 153\end{array}$

$\begin{array}{ccccc}4-15 & 6 & 101 & 125 & 18 \\ 5 & 6 & 87 & 93 & 16\end{array}$

$6-156111,10322$

8.15 6. 64.6716

$8-15,6,64,97,16$

$10-15,6,162,161$, 38

$1215 \% 6.96 \% 9$

$\begin{array}{cccccc}13 & -15 & 6 & 89 & 99 & 16\end{array}$

$-5-14,6,30,22,30$

$\begin{array}{lllll}4-414 & 6 & 32 & 58 & 31 \\ -3-14 & 185 & 187\end{array}$

$2-146$ 6 63 \% 39

$-1-146,30$, 29

$0-14,6,140,132,10$

$\begin{array}{lllll}1-14 & 6 & 69 & 87 & 22 \\ 2-14 & 6 & 0 & 29 & 1\end{array}$

$\begin{array}{llll}2-14 & 6 & 0 & 29 \\ 3-14 & 6 & 269 & 261\end{array}$ h l lopo lope $10 \mathrm{a}$

8.16 16515

10.16 o 102

13.16 o 15

-7.1 \% 10

.1 11 os 37

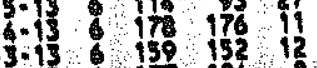

$2.13617906 \%$

$0.136{ }^{6}, 0,321$

2.13612011310

$3-1362119202$ 15

$5 * 13 \quad 11212119$

6.136163 167 24

613610469

$0.136,37,6537$

$10-136 \% 2011^{6}$

12.136 o 10726

$13.13 \quad 60 \quad 6719$

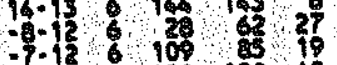

- $-12 \quad 1200$

- -1262032627

- - $12 \quad 6 \quad 108$ 16 15

$-2-12623251$

0.126100 क 63

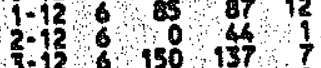

$3.12,6 \quad 150$ 137 ?

5.12615515614

$6-12610210018$

$8-126110109$

$10-12 \quad 16016211$

$1=12,62216$

$12-12,6$ 201 200 15

$16-1269365$

$-9.1161031003$

$-8-116,57,13 \quad 15$

$-7.116109110$

$-6.116213 \quad 18912$

- $5-916$ 214 211

$-3-116357359$

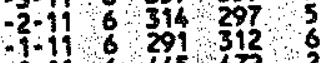

$0-116$ 45 472 ?

$1-11605 \% 91$, 11

$\begin{array}{llll}2-11 & 6 & 87 & 83\end{array}$

$4-116385$ 381 7

$5.116 \quad 65 \quad 7 \quad 32$

$6.116247 \quad 235$

$\begin{array}{lllll}7-11 & 6 & 232 & 229\end{array}$

$8.116 \quad 60 \quad 6923$

$\begin{array}{lll}9-11 & 6 & 113 \\ 0-11 & 663 & 262\end{array}$

$11-196 \quad 20026$

$13-116 \% 776715$

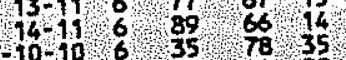

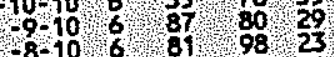

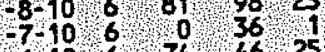

$6-10 \times 6,74,4625$

$\begin{array}{ccccc}-5 & -10 & 6 & 152 & 137 \\ -5-10 & 10\end{array}$

$3-10,624028$

$-3-10 \times 6$ 6 149 148 ,

$79106403 \quad 391$. 6

$0.106,105 \% 93 \quad 14$

$\begin{array}{ccccc}1-10 & 6 & 103 & 95 \\ 2-10 & 6 & 248 & 265 & 8\end{array}$

$\begin{array}{llll}2-10 & 6 & 248 & 265 \\ 3-10 & 6 & 242 & 242\end{array}$

$\begin{array}{llll}4-10 & 5 & 81 & 78 \\ 5-10 & 6 & 212 \quad 208\end{array}$ $h$ 1 10po 10pe $10 \mathrm{~s}$

6.10 13

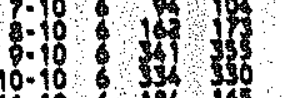

$1.10 \quad 61267$

$13=10$ 14 13

$16-10 \% 02109$

$10=96123151$

-9 9167 15 is

-8 $: 9 \quad 71918 \%$

$\% 066261283$

$306027 \% 269$.5

106270276

1.0623521

3.96641345

6.96504 69

6.96498

8.96241635

9.9189189

10.9615 \% 17

$12-9613600$

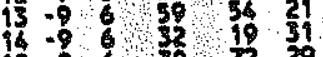

$12-6.6 \quad 30 \quad \frac{27}{45}$

$10-60516351$

-86193159 10

$-7.86272697$

$-6-86$ 615 120 25

- 8616190124

-2.8 6245246

$-1-8161 / 175$

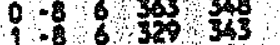

$2-8662,454,1$

3.86335515

$\begin{array}{ccc}6.8 & 6 & 339 \\ 5 & -8 & 160\end{array}$

$6-86197,183$

$7-86217224$

$8-86181$ i 65

$10-86108,108 \quad 10$

$\begin{array}{ccc}11 & -8 & 6\end{array}, 43,41,15$

$\begin{array}{lllll}13 & -8 & 6 & 0 & 70 \\ 14 & -8 & 6 & 14\end{array}$

$\begin{array}{rrr}14 & -8 & 6 \\ -13 & -7 & 6\end{array} 0^{0} 34$

$-12-7.6450,6349$

$-10-7623521715$

$-8-76133131 \quad 15$

$-7-7610312115$

$\begin{array}{ccc}-6 & -7 & 6186193\end{array}$

$-4-7 \quad 628529$

$-2-7 \quad 6 \quad 417 \quad 419$

$-1-76384383$

$1.7 .6 \quad 3494410$

$2-76393 \quad 425$ 11

3.7. 6.811813

$5.76423,462$

$\begin{array}{lllll}6 & 7 & 6 & 219 & 232 \\ 7 & 626\end{array}$

$\begin{array}{llllll}8 & 7 & 6 & 102 & 97 & 10\end{array}$

$10776205,202,4$

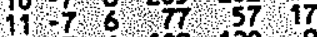

$\begin{array}{llllll}12 & 7 & 6 & 109 & 120 & 9 \\ 3 & 7 & 6 & 33 & 51 & 33\end{array}$

$14-76 \% 73$ 84 17

$\begin{array}{cccccc}-13 & -6 & 6 & 91 & 74 & 43 \\ & -6 & 6 & 46 & 181 & 20\end{array}$

$\begin{array}{llllll}-11 & -6 & 6 & 193 & 181 & 20 \\ -10 & -6 & 6 & 145 & 122 & 1\end{array}$ 


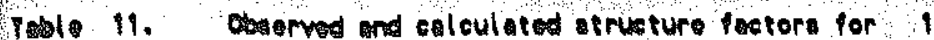

in 1 10Po 108 108

- 132 159 12

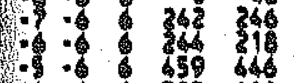

10

- : 5150

9.6 5797

3.6 3007300

1. 6 - 535

1.6353

$1.660^{3} 3$

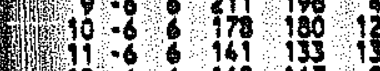

12.6169167

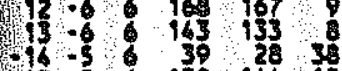

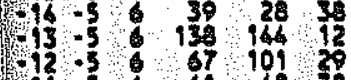

m.

- .5 6071910

$\begin{array}{ccc}-6.5 & 603 \\ -7.5 & 3\end{array}$

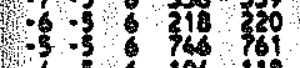

$\begin{array}{lll}-4-5 & 6 & 105 \\ -3 & 5 & 5\end{array}$

- .5 110 11 13

-1.5 313 224

$\begin{array}{lll}1 & -5 & 5\end{array}$

$4.5 \quad 39261$

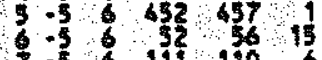

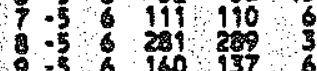

$10-5 \quad 6 \quad 140137 \quad 6$

$\begin{array}{llll}11 & 5 & 6 & 142 \\ 13 & 5 & 39 & 37\end{array}$

-15 601025

$-12-6 \quad 60163$

$\begin{array}{llll}-11 & -6 & 75 & 81\end{array}$

$\begin{array}{lll}10-6 & 162 & 152\end{array}$

$\begin{array}{llllll}-8 & -6 & 6 & 325 & 325\end{array}$

$\begin{array}{llll}-6 & -6 & 6 & 62307 \\ -5 & -4 & 6 & 205\end{array}$

- $-6 \quad 569531$

$\begin{array}{lllll}-2 & -4 & 6 & 399 & 390\end{array}$

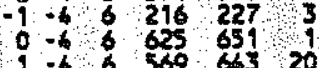

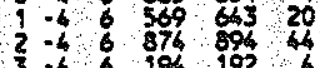

3.6619192

$\begin{array}{lllll}6 & -4 & 6 & 247 & 258 \\ 5 & -4 & 6 & 399 & 388\end{array}$

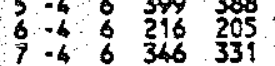

$\begin{array}{llllll}7 & -5 & 6 & 346 & 331 & 5 \\ 8 & -6 & 6 & 163 & 164 & 12\end{array}$

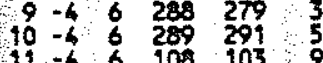

$12-6 \quad 108104 \quad 21$

$13-4613312717$

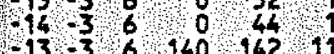

$\begin{array}{rrrrrr}13 & -3 & 6 & 140 & 142 & 11 \\ 1 & -3 & 6 & 95 & 164 & 10\end{array}$

$-10-3,6,169,166,21$

$9.36330 \quad 334$

$\begin{array}{llll}-7 & -3 & 691 & 490 \\ -7 & 3 & 222 & 229\end{array}$

$\begin{array}{lllll}-7 & -3 & 6 & 222 & 229 \\ -6 & -3 & 401 & 527 & 5\end{array}$

$-5-3,6433,508,4$

$\begin{array}{lllll}-6 & -3 & 6 & 532 & 543 \\ -3 & 602 & 601\end{array}$

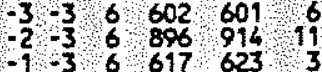

\begin{abstract}
h 1 1 1070 10\%e 100
\end{abstract}
- $\quad 697 \quad 693$

\% : 8410010

- 197164

10.3178167 - 15

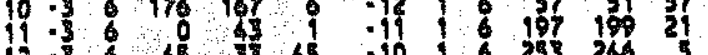

$12=6565$

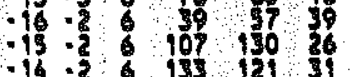

$-16=3$ 131

-1) $8016 \%$

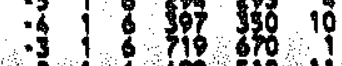

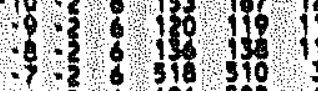

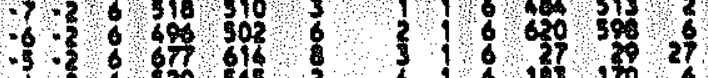

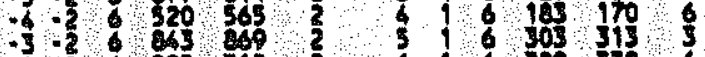

$-2.26293 \%$

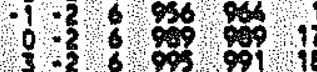

3. $6095 \%$ \% 6

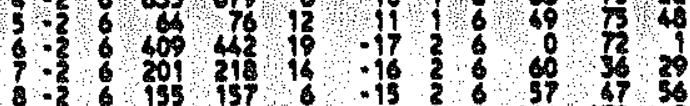

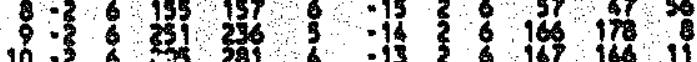

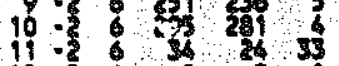

$-16=8 \quad 150 \quad 17012$

$-13=614919016$

$-12-16168158$

$-10-16403391$

$-1671060$

$-7-160067$
$-6-16065$

$-5-16$ st

$-1.6706$

$-1.1670967$

- 8 675

165458917

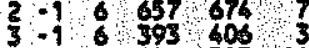

$\begin{array}{lll}3 & -1 & 693 \\ -1 & 6525 & 53\end{array}$

$-1626836$

$7-16300326$

$8-6177170$

$10-16130111 \%$

$\begin{array}{lllll}-17 & 0 & 6 & 61 & 60 \\ -16 & 0 & 6 & 39\end{array}$

- 5 o $6285 \quad 29215$

- 13.0678

$\begin{array}{lllll}-12 & 0 & 6 & 184 & 183 \\ -11 & 0 & 6 & 172 & 186\end{array}$

$-10 \quad 0 \quad 6 \quad 170 \quad 18618$

$\begin{array}{lllll}-9 & 0 & 6 & 242 & 223 \\ -8 & 0 & 6 & 607 & 613\end{array}$

$.706,206,195,8$

${ }^{-5} 50611651206,4$

$4.0,35,15 \%$

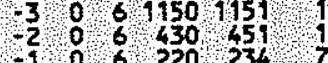

$\begin{array}{lllll}1 & 0 & 6 & 220 & 234 \\ 0 & 661 & 501\end{array}$

10.6665 611 21

$2006 \% 172,180,8$

$4006469,448 \quad 10$

$5006,225,219,4$

$\begin{array}{llllll}6 & 0 & 6 & 316 & 317 & 4 \\ 7 & 0 & 6 & 174 & 165\end{array}$
- 12 o 197109

-10 2027049

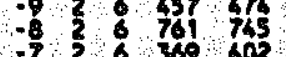

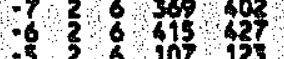

-3 210712511

- 2631616

${ }_{0}^{1} 261025$

$\begin{array}{lll}1 & 2 & 516 \\ 3 & 501\end{array}$

$3 \quad 6 \quad 167 \quad 157$

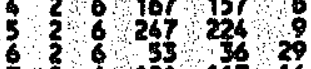

$7 \quad 6121$ 19 16

926211005

$\begin{array}{ccccc}90 & 6 & 211 & 205 & 50 \\ -10 & 2 & 6 & 65 & 55\end{array}$

$.18 \quad 3 \quad 6 \quad 72 \quad 45$ 3

$\begin{array}{lllll}-16 & 5 & 63 & 11 & 62 \\ -15 & 3 & 221 & 222 & 6\end{array}$

$\begin{array}{llll}-16 & 3 & 6 & 32 \\ -13 & 33 & 32\end{array}$

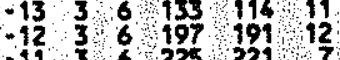

-9 36263267

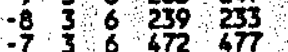

-6 3 - $61171 \quad 1221 \quad 15$

$\begin{array}{lllll}-4 & 3 & 6 & 378 & 350\end{array}$

$\begin{array}{llllll}-4 & 3 & 6 & 01 & 026 & 2\end{array}$

$\begin{array}{lllll}-7 & 3 & 6 & 317 & 287 \\ -1 & 3 & 6 & 486 & 479\end{array}$

$\begin{array}{lllll}1 & 3 & 6 & 686 & 489 \\ 0 & 3 & 6 & 298 & 289\end{array}$

$\begin{array}{lllll}1 & 3 & 6 & 353 & 332 \\ 2 & 3 & 655 & 351\end{array}$

3,36273 220,

$\begin{array}{llllll}4 & 3 & 6 & 170 & 165 \\ & 3 & 6 & 0 & 20\end{array}$

$\begin{array}{llllll}6 & 3 & 6 & 93 & 95 & 23 \\ 7 & 3 & 6 & 303 & 311\end{array}$

836167 161, 8

$\begin{array}{cccccc}9 & 3 & 6 & 35 & 44 & 35 \\ 10 & 3 & 6 & 141 & 113 & 10\end{array}$

$-184462314422$

$\begin{array}{lllll}17 & 4 & 6 & 87\end{array}, 87,30$

$-1646,96,7821$
- $\quad 6 \quad 5095$

$-11362252211$
-15.16155 का 55

$11-16 \% 98 \%$ h I lopo lope 10a

11 $13013 \%$

. $\%$ \& $3010 \%$

-7 6975

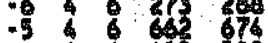

-5. 402508

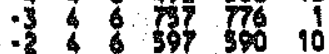

-1 6.195

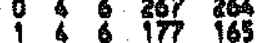

- 21705

18 3 35

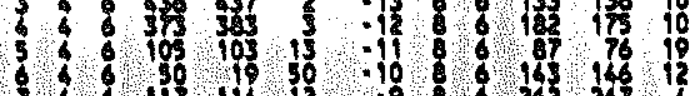

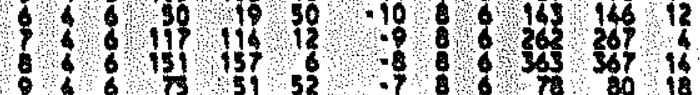

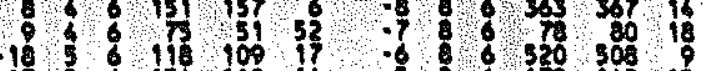

(1) 613 in

-15 5 \% 14413 ?

$135.5165,152,26$

1256.163745

$-1058269297$

- 6.353

$-756469$

- $5 \quad 6 \quad 375$

$\begin{array}{llll}-4 & 6 & 350 & 304 \\ -5 & 6 & 445 & 431\end{array}$

1 . 60 .

0 s 615618

3 $5{ }_{119} 160$

$5 \quad 613514310$

$6 \quad 61719180$

- 6179166

$3660^{\circ} \quad 201$

$-16 \quad 6 \quad 61316612$

- 617207

$\begin{array}{llll}16 & 6 & 203 & 108\end{array}$

$\begin{array}{llll}-12 & 6 & 6 & 0 \\ -11 & 6 & 6\end{array}$

$-106635352$

$\begin{array}{llll}-9 & 6 & 6 & 265 \\ -8 & 6 & 6 & 37\end{array}$

-7 66734750

$\begin{array}{llll}-6 & 6 & 6 & 257 \\ -5 & 6 & 6 & 219 \\ & 6 & & 218\end{array}$

- $6 \quad 6 \quad 6 \quad 219 \quad 2182$

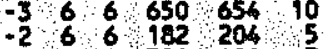

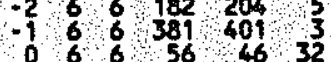

$46.152,143$

$26 \quad 6 \quad 142 \quad 129$

$\begin{array}{llll}3 & 6 & 6 & 277 \\ 4 & 285\end{array}$

$\begin{array}{llllll}5 & 6 & 6 & 101 & 100 & 10 \\ 5 & 6 & 6 & 248 & 227 & 9\end{array}$

$6.695 \quad 7816$

$\begin{array}{lllll}7 & 6 & 6 & 121 & 136 \\ 8 & 6 & 12\end{array}$

$-197600991$

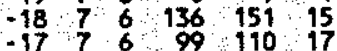

$-167.6285260 .6$

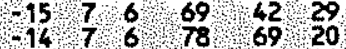

13.7.6 78,247

$-1176,68$ 20 27

$-1076,120,12012$

$876,306,342,5$

$\begin{array}{llllll}-7 & 7 & 6 & 306 & 392 \\ -6 & 7 & 6376 & 358 & 4\end{array}$

$\begin{array}{lllll}-6 & 7 & 6 & 376 & 358 \\ -4 & 7 & 6 & 752 & 74\end{array}$ h l lofo 10\% 100

1) $13 \%$ of

$\$ 76160$

7761213610

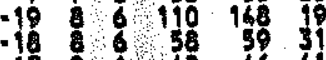

-17 6162060

15 . 109 क 10

-16 635 35 6

-6.8\%350 300

-6. 623624

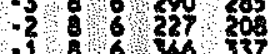

-186 628 है। 1

1.6\%200 249 19

3. 6161967

$566^{636} 2313$

.6865237

-18 $6 \% 51$ 45 50

$-17: 610211022$

$-15.62113515$

-13
-12

$-10862436$

.96230217

$.7 \quad 6 \quad 22030$

- 696269237

- $96187 \quad 174 \quad 2$

-296160110 is

$-19625261 \quad 6$

196105 202 ?

36707018

59610016211

$\begin{array}{lllll}-18 & 10 & 6 & 54 & 39 \\ -17 & 10 & 64 & 78\end{array}$

$-16106058763$

$-1510616416010$

$-13106326315$

-1110687 83 86

$-1010,6164,151 \quad 9$

$-8106306319 \quad 7$

$\begin{array}{lllll}-7 & 10 & 6 & 260 & 269\end{array}$

$\begin{array}{llllllllllll}-6 & 10 & 6 & 157 & 144 & 18 \\ -5 & 10 & 6 & 222 & 208 & 5\end{array}$

$\begin{array}{llllll}-2 & 10 & 6 & 106 & 119 & 14 \\ -1 & 10 & 6 & 109 & 109 & 17\end{array}$

$0106 \% 98 \quad 86 \quad 15$

$1106,122,119$, 15

3106.165163 ?

$-19196,102,96,12$

$-18116,132,127 \quad 12$

$-16116241.21831$

$\begin{array}{llllll}15 & 11 & 6 & 31 & 45 & 39\end{array}$

$-13116271273 \quad 6$

$-11116{ }^{6} 127$ 123 16 $\begin{array}{llllll}-4 & 10 & 6 & 122 & 126 & 11 \\ -3 & 10 & 6 & 56 & 66 & 30\end{array}$ 


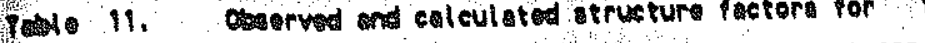

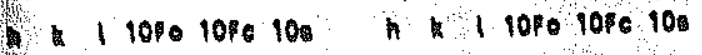

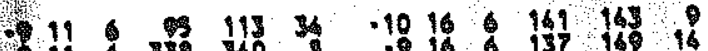

711 19 100

$11 \%$

४ง 19

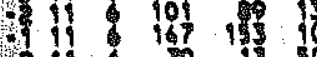

11 5

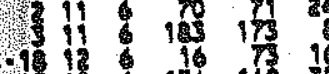

41 is 13190

43617 10 $110176 \%$ की

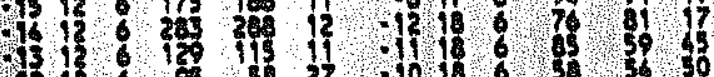

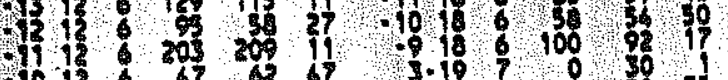

1012 6 01017

\% 12 \% 315 ?

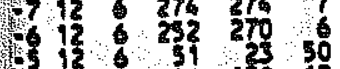

412,15130

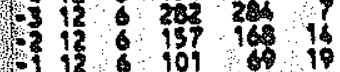

11 1210120

यद 17 19

97 is 14 10\%

1513 \% 155160

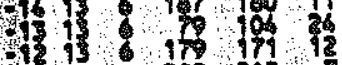

111020212

o $13.1^{136} 140$

-7 13 11 971

-1 113 12 1

- $13 \quad 12 \quad 10016$

- 13161010

- 1 197 का 92

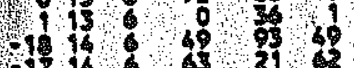

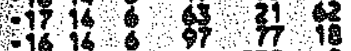

15 19 1251232

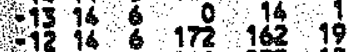

If $16 \quad 26123512$

$-1916 \quad 208205$

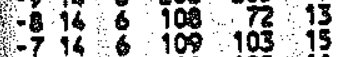

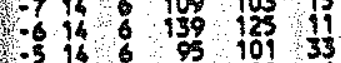

.5166 क 90

- 36.609 \begin{tabular}{cccccc}
-2 & 16 & 6 & 75 & 86 & 35 \\
-1 & 16 & 5 & 129 & 122 & 23 \\
\hline
\end{tabular} $-1713 \quad 6 \quad 40 \quad 4359$

$\begin{array}{rllll}-16 & 15 & 6 & 156 & 158 \\ -15 & 15 & 6\end{array}$

$\begin{array}{rrrrrr}16 & 15 & 8 & 67 & 19 & 31 \\ -13 & 5 & 129 & 120 & 16\end{array}$ $\begin{array}{llll}13 & 6 & 206 & 21710\end{array}$ $1215 \quad 6 \quad 244253 \quad 13$ $-101569164016$ $-915 \% 6$ 16 60 \%

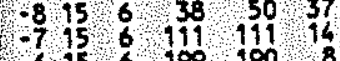

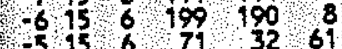
4156137 134 15 -2 $15 \% 71 \% 7626$ $\begin{array}{llllll}-16 & 16 & 6 & 103 \\ -15 & 113 & 15\end{array}$ $\begin{array}{rllll}-159 & 6 & 71 & 75 \\ -14 & 69\end{array}$ $1316 \quad 614796313$ 1216,6217211 \% 90 1. 197,3035 $5=1976787$ 7.197 \% 38

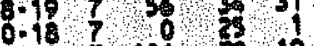
1.18 ? 109 \% 19

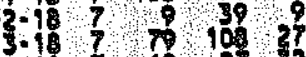
5.19 7137 ? \%.1 7 113 8. 7 ? 12 $10.1900 \% 16$ -197 1721027 17117 13 l? 3.17 7 , 750 4-17 74301 $\begin{array}{lll}5.17 & 7 & 100\end{array}$ 7.77 . 13 $9.17 \% 13$ 3 $90.17,7$ क का की -3.167 195 b3 27 $-2-167$
-113 0.1670155 2-16 7 क 1619

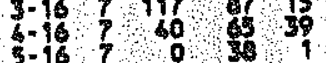
$6.167,76$, 35

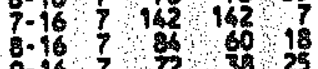
$9-1677723$ $10-16,7$, 56 , 39 ? $12-167107119 \% 2$ $-4-157 \quad 64884$ $\begin{array}{llll}-3-15 & 7 & 36 & 32\end{array}$ $\begin{array}{llll}-2-15 & 7 & 85 & 40\end{array}$ $\begin{array}{rrrr}-1.15 & 7 & 70 & 62 \\ 0.15 & 7 & 213 & 218\end{array}$

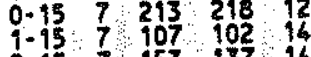
$\begin{array}{lll}2-15 & 753 & 93716 \\ 3 & 75 & 171\end{array}$ -15 7208200 5. 5 ? $\quad 26 \quad 139,16$ $6.5,7,165$, 906 ? 8. 15,7 . 85, T5 1313 $0.157,110$ ?6? $19-15 \div \quad 165$ 171 6 $12.15 \% 7 \quad 122,133 \quad 1$ $=6.14,7$, 06 $-4-147$ ? 104 \% 8119 $-3-14,7,194,212,10$ $-1.14 \quad 7 \quad 246 \quad 254 \quad 6$ h. I 10\% 10Fe 100

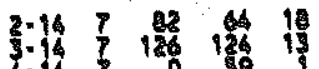

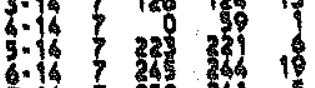
6.14 7350 8.167200 10.16790 12:18 ? o .7. 7 o 015 - -977 is 100 -3.157 201 20 15 - 75757 $0: 13,236$, 2. 3161019 ร.13 7 121 13 7.13 7 16 2 - 1375163 10.137163 का

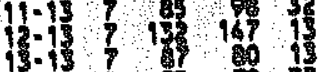
- 7 \% 7 त 5 - . 7 .

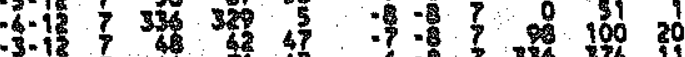

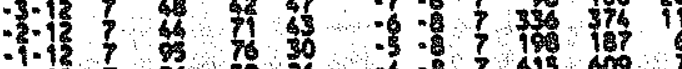
$\begin{array}{ll}1.12 & 727 \\ 0.12 & 7\end{array}$ 1.12 $72835 \%$ $\begin{array}{lll}2.12 & 7 & 0\end{array}$ \$.12 723412311

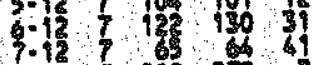
6. 7257 10.12 7 , 145,139 $12-12745120$ -97 ? 73 $=-117 \% 10010$ -6-117 74716914

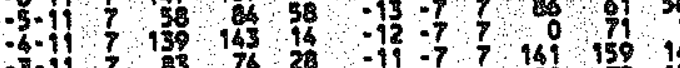

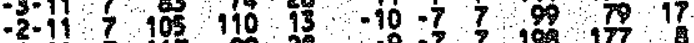
$-1.117117,99$ 1-11 735 -331 2. 197256255 $3.11,7199198,6$ 5-11 7113 \&5 17 $\begin{array}{lllll}6-1 & 7 & 188 & 188 \\ 7-11 & 7 & 193 & 185\end{array}$ $8-11 \quad 7 \quad 175$ 158 5 $\begin{array}{ccccc}9-11 & 7 & 24 & 54 & 23 \\ 0.11 & 7 & 222 & 217 & 4\end{array}$ $19-117 \% 8379$ $22-197 \quad 17 \quad 44 \frac{17}{3}$ $-10.907 \quad 30 \quad 21 \quad 30$ $-9-10,7179,164,18$ $-7.107119109,23$ $-6 \cdot 10 \quad 7,118 \quad 136$.24 $-4-107,302,302,5$ $-3-10,72462475$

$-2-107120,14414$ -1.10
0.10 $1-107,138,141$,

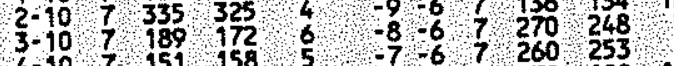

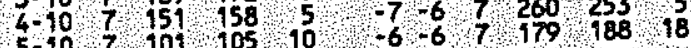

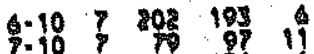
\% 11.10 7 ? 50 1.:9 7 \%

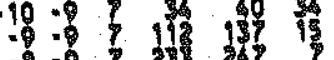
-7.97 蜘 13

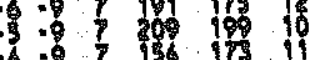
- 19 iा

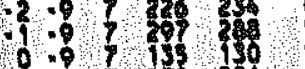
3 -9.91019 5 719 145

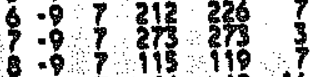
907 - 6016 $11.97 \%$ का 15 $13=97 \quad 70^{7}$ $-67613$

- -27576

$0-127276$

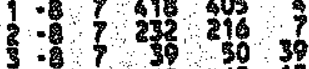

- 72000

7. 72 272

- 1 . 30190

$10-173936$

$11-72006$

$12-71091606$

$-13-77136$

-9 - $7 \quad 7 \quad 198177$

$\begin{array}{lllll}-7 & -7 & 7 & 260 & 240 \\ -7 & -7 & 7 & 5 & 51\end{array}$

$6-7 \quad 710711912$

$\begin{array}{llll}-4 & -7 & 7296 & 291 \\ -5 & -7 & & \end{array}$

$\begin{array}{llllll}-2 & -7 & 7 & 86 & 100 & 12\end{array}$

$\begin{array}{lllll}1 & -7 & 7 & 551 & 325 \\ 0 & -7 & 7 & 273 & 285\end{array}$

$\begin{array}{lllll}1 & -7 & 7 & 100 & 101 \\ 2 & -7 & 7 & 300 & 314\end{array}$

$\begin{array}{lllll}2 & -7 & 7 & 396 & 381 \\ 4 & -7 & 7 & 217 & 225\end{array}$

$\begin{array}{lllll}5 & -7 & 7 & 166 & 177\end{array}$

$\begin{array}{llll}6 & -7 & 723 & 216\end{array}$

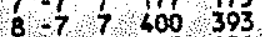

$9-7,7425 \quad 421$

$\begin{array}{llllll}10 & 7 & 7 & 148 & 157 & 6\end{array}$

$12-7,767 \quad \frac{5}{5}, 15$

$\begin{array}{lll}13 & -7 & 75\end{array}, 46 \quad 20$

$\begin{array}{llllll}-13 & -6 & 7 & 68 & 84 & 67\end{array}$

12.6710596017

$10-67167$

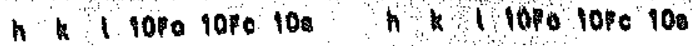

$0.14782,62,35$
- 1 O A

$-1030$

1.6740

3. $.6 \quad 7 \quad 300$

-6 713151

- . 7165

8.7250230

$10.6105 \%$

12.67 . 97 \%

13.671710017

13.571919

10.731510

.-5710 ig 16

-7.5169 is 10

.6 .57925

- 57804

- "3 165267

- 1.5724250

0.7739

2.7746

- 74509

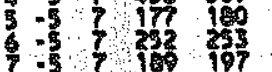

- $-7,1210$

9.57 1410

$11-5723152$

12-5? 75 \% 69

- $15-4,7,10131$

$-13-6720 \quad 232$

$-11-4726020515$

$0=75595535$

$-8.4714153$

$-6-476762$

-5 - $7510 \quad 522$

$-3.674354$

-2 -618040

$0-47256260$

1.47323301

$5-67321,330$

6 -4 7267 265

$8.47 \%$

$9+47211 \quad 205 \quad 5$

$10-47,65 \quad 62 \quad 16$

$12-4 \quad 7160176$

$\begin{array}{llllll}15 & -3 & 7 & 26 & 60 & 26 \\ 14 & -3 & 7 & 146 & 150 & 11\end{array}$

$\begin{array}{llllll}-13 & -5 & 7 & 0 & 28\end{array}$

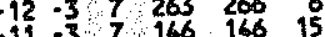

- $10-3 \quad 7261262$

$-9-377181 \quad 154$

$-8.5 \% 325333$

-6.3. $579555 \%$

$\begin{array}{lllll}-5 & -3 & 755 & 488 \\ -4 & -3 & 546 & 560\end{array}$

-3 . 7 761 80710

$-2-37718718$ 6

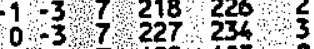

$\begin{array}{llllll}403 & 7 & 425 & 403 & 8\end{array}$

$6 \% 3,7282$ 91??

$\begin{array}{llll}13 & 3 & 742 & 91 \\ 90 & 9\end{array}$
1.75 



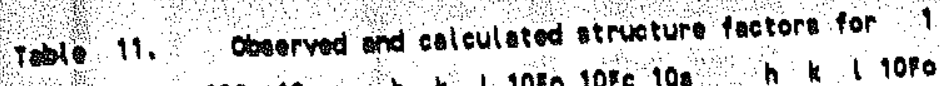

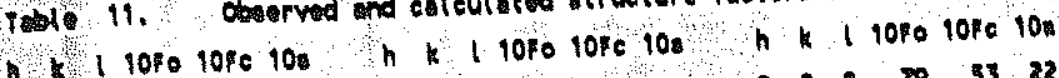

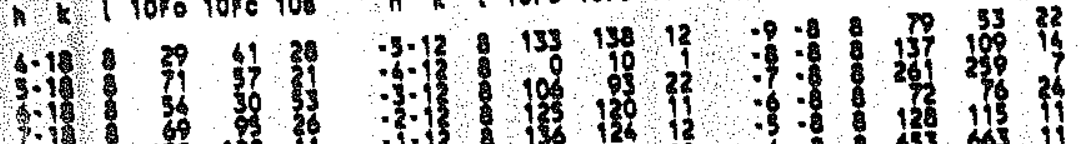
a.1. 13: 128 7. 0.17 .1313139 $177.62,60.4$ S17 107100 517 . 150 153 797110 196 16 9.1785195 10-17. 8 5 1.1812012310 0.96 . $107 \quad 11714$ 2.19 103104 is 3.16 190 10 38090 610 of 26 6-1 916 $10.16 \quad 1390137 \quad 30$

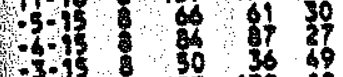

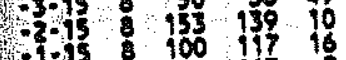
$0-5$ is 1319 215 2909 $375 \quad 237 \quad 26010$ 615 1051001

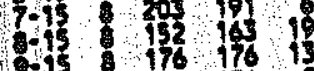
$10-15$ 13 170

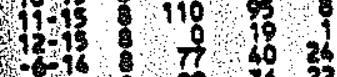
4.16 क 76 क 32 616 7972032 1.16891312310 1.16 $\quad 102 \quad 107 \quad 17$ $\begin{array}{lllll}216 & 8 & 1907 & 72\end{array}$ 414 1260 148 10 Q16 $80^{53}{ }^{363}$

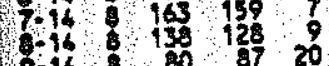
9014,808720 $10-16$ के 710

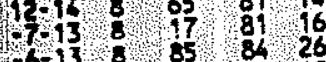

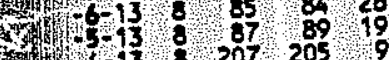
413 8 $207,205 \% 1$ 12.138213208 . 6 01389911913

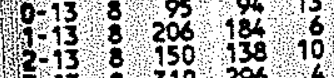
\begin{tabular}{lllll}
13 & 13 & 310 & 296 & 4 \\
\hline-13 & 830 & 227 & 5
\end{tabular} $\begin{array}{lllll}5-13 & 8 & 79 & 73 \\ 7 & 209 & 2217 & 85\end{array}$ $80.13 \quad 230 \cdot 229 \quad 4$ $\begin{array}{rllll}90-13 & 8 & 188 & 191 & 6\end{array}$ $\begin{array}{rrrrr}10-13 & 8 & 180 & 48 & 12 \\ 2-13 & 8 & 89 & 72 & 12\end{array}$

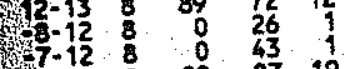

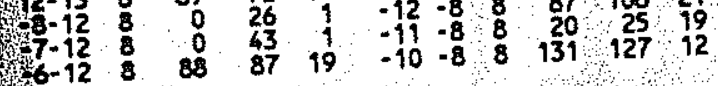

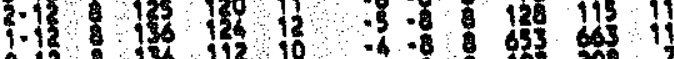
0.12 o $115 \%$ $2.12811012 \%-53$

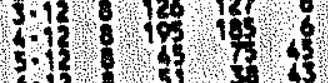

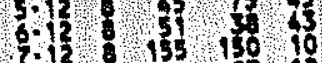

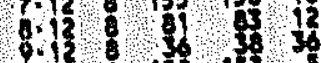

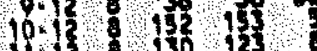
$12.1280,353$ 10.11893035 an1. 1032153 7123020112737

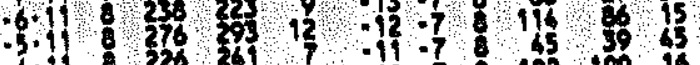

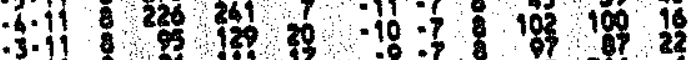

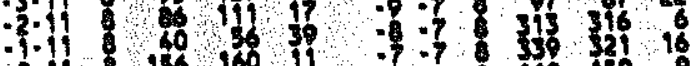
0.1913616011

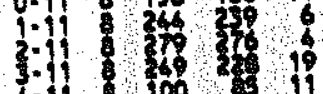
- -1100 का

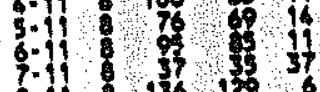
90.18157 10.11 \% 100 $13=1100010$ -90.10 8 39,131 ह $-8.90813015$ $.7-10$ 13 1018 - =10 140161

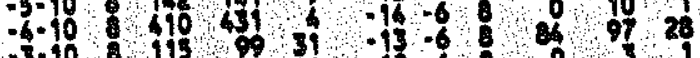

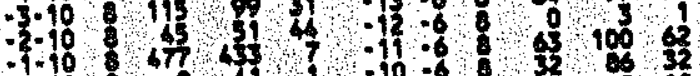
$\begin{array}{rl}1.10 \\ 0.10 & 6\end{array}$ $1.10889297 \% 10$

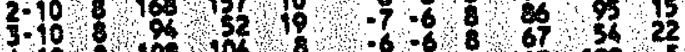

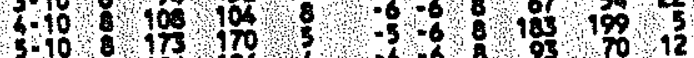
6.108186196 $7 \times 1089195 \%$ 9.10 .134956 $12-10$ 8 83016,10 $12-108 \% 7269$ $\begin{array}{rrrrr}-11.9 & 8 & 0 & 41 & 13 \\ -10 & -9 & 8 & 6 & 52\end{array}$

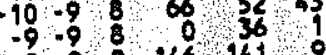
$-7.9 .8,166,141$, 2018 $6.98224219,6$ 4.96116 , 144,5 $\begin{array}{lllll}3 & 986 & 353 & 1\end{array}$ 1.9.8141 137,10 $\begin{array}{lllll}0.9 & 8 & 116 & 130 & 10 \\ 9.9 & 833 & 281\end{array}$ $\begin{array}{lllll}209 & 8 & 254 & 273 \\ 3 & 9 & 122 & 118\end{array}$ 4.9. 8.45 39 , 31 $5.98 \% 230218$. 7.9. 8. 215 195 $8.98 \% 159 \times 151$ \% 10 -9 8 194 1914 $11=9 \quad 65 \quad 42 \quad 27$ $\begin{array}{lllll}12 & -9 & 85 & 42 & 27\end{array}$

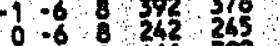

-6 8274284

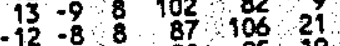

$-6-7 \quad 100150$

-6.7 1121

- $-7 \quad 20101$

-1 -7 20

$1-7 \quad 252$

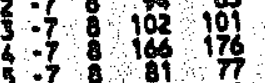

$\begin{array}{ll}6.7 & 122122\end{array}$

- $-7 \quad 260$ रा

19.71313

$177 \frac{17}{25}$

7.276253

$9-3116$ a 232

$10-3.6353$

$-16-200016$

$\begin{array}{lll}-15 & -2 & 8 \\ -14 & -2 & 8\end{array}$

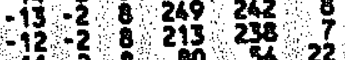

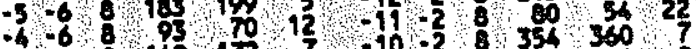

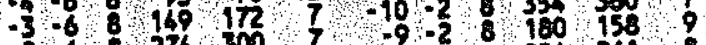

-2.6 627600

$1.6 \quad 611392$

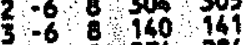

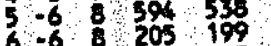

$\begin{array}{ccccc}6 & -6 & 8 & 205 & 199 \\ 7 & -6 & 8 & 275 & 273\end{array}$

$8-6 \quad 8127,123$

$960 \% 213 \quad 206 \quad 19$

$11.68109 \times 116 \quad 12$ -1458 8 124,141912

$\begin{array}{llll}-12 & 5 & 8 & 284 \\ 261 & 16\end{array}$

$-91058 \quad 97 \quad 126 \quad 44$

$-9.5,8 \quad 247 \quad 236 \%$

8. 58,854367

$0.5,8449 \quad 436$

2.58398428

$3-58016 \quad 58414$

$\begin{array}{lllll}-2 & -5 & 8 & 231 & 250 \\ -1 & -5 & 8 & 142 & 138\end{array}$

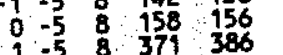

$\begin{array}{lllll}1 & -5 & 8 & 372 & 306 \\ 2 & -5 & 8 & 242 & 230 \\ 5 & -5 & 8 & 232 & 243\end{array}$

$\begin{array}{lllll}5 & -5 & 8 & 232 & 24\end{array}$
$-8-2 \quad 825261$

$\begin{array}{lll}-7 & -2 & 824\end{array} 60$

$\begin{array}{llll}-5 & -2 & 239 & 267\end{array}$

$\begin{array}{lll}-4 & -2 & 89 \\ -3 & 20 & 29\end{array}$

$\begin{array}{llll}-2 & -2 & 830 & 355 \\ -1 & 256 & 262\end{array}$

$\begin{array}{ccc}-1 & -2 & 8 \\ 0-2 & 809 & 259\end{array}$

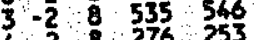

$4-2 \quad 8276$

$6.28130 \quad 130$

828170174

$10.28 \%, 0$, 13,12

$\begin{array}{ccccc}11 & -2 & 13 & 21 & 12 \\ -16 & 8 & 85 & 26\end{array}$

$\begin{array}{rlllll}-15 & -1 & 8 & 231 & 254 & 6\end{array}$

$-13-28 \times 11211518$

$-11-188345 \quad 352$ 8

$-10.188210,193,14$

$\begin{array}{llllll}-9 & -1 & 8 & 257 & 574 & 6 \\ -8 & -1 & 8 & 571 & 574 & 6 \\ -7 & -1 & 8 & 124 & 124 & 20\end{array}$

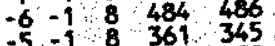

$\begin{array}{lllll}-5 & -1 & 8 & 3618 & 332 \\ -4 & 318 & 3\end{array}$

$\begin{array}{lllll}-3 & -1 & 8 & 362 & 335 \\ -2 & -1 & 8 & 48\end{array}$ i lopo lofe 100

i: 1 \% 1900 ?

: : 12 19 19

.1 812300

- 1716

- $\quad 330337$

610137122

$1608 \%$ \% 101

14082420012

12.0207 21 12

10. 0842 का 3

.8066167310

$.70860 \%$ ip

-5 083903512

- 0 of 60

- 20 \% $\%$

- 8115109 13 $^{13}$

20000 की

10142145

6011103

o 5050

0 0 030

- 19 \& 100 \& 16

-16 130116

- $16 \quad 8200202$

-13 12 17 10

$-11 \quad 300300$

$-918666412$

$-8 \quad 8140,13819$

\begin{tabular}{l|lll}
-6 & 8 & 893 & 53
\end{tabular}

-5 1837360

$-318575002$

-2 1426437.6

- 8454007

218216225

31009

$51 \quad 8123121$

718132,132

$818 \% 03$

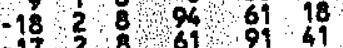

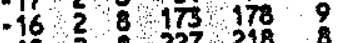

$15 \quad 2 \quad 8 \quad 227$ 218 8

$-13,2$ 8 164 14610

12 2. $151,134,19$

10 2 $8321 \quad 303$ प

8 2 8 381 384 3

-628345301 4

$-528566,54610$

$\begin{array}{llll}-4 & 2 & 8 & 202 \\ -3 & 223\end{array}$

-2 2 81290 274

1. 2 8 171 173 4

2 $288147 \quad 167,6$

328456221

$528177156 \quad 5$

$\begin{array}{llll}6 & 2 & 0 & 2134 \\ 7 & 8 & 136 & 8\end{array}$ 


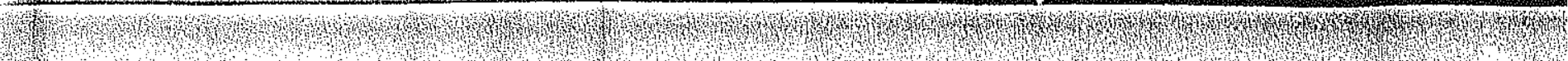

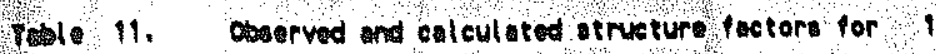

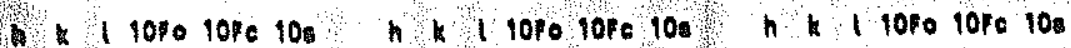

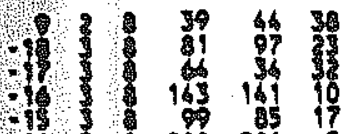
प1 ह ? 2010 \% 17109 47 5 35 (2) 13 C. 3 392 303

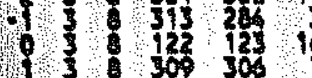

13 o 300

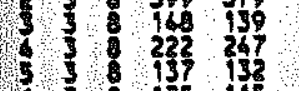

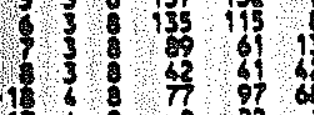

1

$19 \quad 18101$

11 230 230

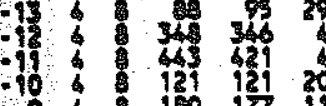

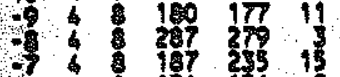

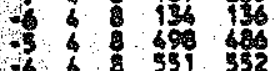

$\begin{array}{lll}6 & 5152 \\ 3 & 200 \\ 0 & 246 & 320\end{array}$

$1 \% 13$

1 . 190

1017

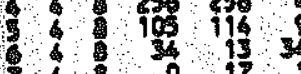

$17 \quad 12017$

$19 \quad 29520$

- $15 \quad 5 \quad 100 \quad 39$

$-13 \quad 1651641$

$-115 \mathrm{~g} \quad 17$

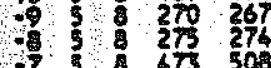

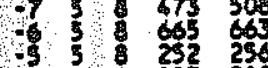

\begin{tabular}{lllll}
3 & 5 & 8 & 252 & 250 \\
-4 & 5 & 223 & 209 \\
\hline 3 & 5 & 8 & 202 & 202
\end{tabular}

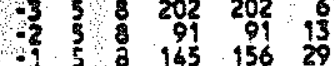

\begin{tabular}{rlllll}
-9 & 5 & 3 & 145 & 156 & 29 \\
\hline & 5 & 310 & 324 & 3
\end{tabular}

$\left\{\begin{array}{lllll}5 & 8 & 303 & 280 & 5 \\ 5 & 8 & 70 & 86 & 21\end{array}\right.$

653304300

5 5. 5122,115

$19680^{2}, 23$

$1868862 \quad 6041$

$176 \quad 6 \quad 8 \quad 260 \quad 257 \%$

$-1568200 \quad 18910$

$1468201 \% 195,13$

1268024091139

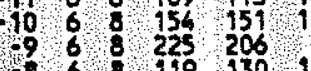

868,4094130

$-6.846344516$

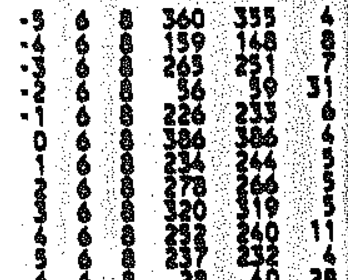

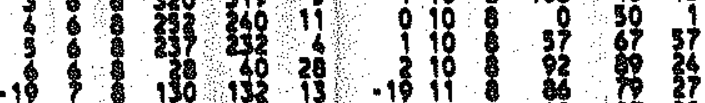
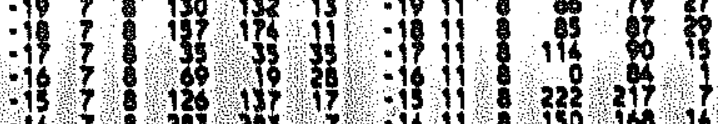

1678252

$-12781651730$

- 078 17 164

- 778590012

- 7867375

$-67139123$

- 7 1601510

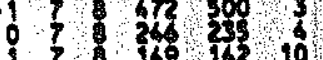

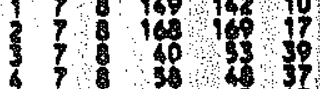

$57 \%$ का

$.19 \quad 220223$

$\begin{array}{lll}-17 & 0 & 0 \\ -16 & 165 & 35\end{array}$

-15820 17

$\begin{array}{lll}-13 & 8\end{array} 19316219$

-11 \% 1212

$.10 \quad 806$ of $^{6}$

- 181619

-6) कर 10

- 80209696

- 8 8 $140 \% 16$

0.807016

38815616313

68807522

$-19.9810311747$

$\begin{array}{ll}-17 & 9\end{array} 513142$

-1598 298276

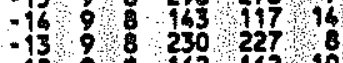

-12, 162162,10

$-11.823199,14$

$.9 \% 8: 292 \quad 308 / 15$

$-8.98 \quad 160 \quad 162$ 12

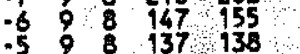

$-4.98207218 \quad 6$

$-398,69,6829$

198,2902272 ह

109815816910

?. $98,174,180,15$

1910 8 10310517

$-1810,8,154,140,12$

$16.10,8,221,212,19$

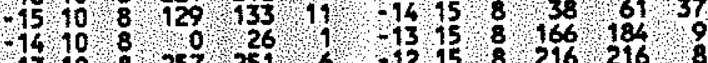

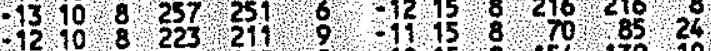

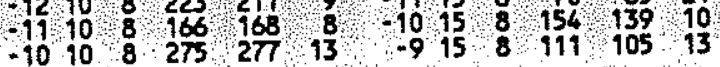

- 118151 161?

$-21619170$

of 100 a -19 12 of 87

$1612 \quad 136$ का 15

-1312 o 3160 \$10

11,8 \% 9716

-9 12 \% 13109

.812812314210

- 12 . 362 ? 212 ?

- $13 \quad 167 \quad 160$

- 12 o 107122 2

$-18138140118 \%$

-17 13 0 का 22

- 15 13 8 213 199

$-1213815312416$

1013825024916

- $\begin{array}{llll}3 & 8 & 0\end{array}$

- $613 \quad 216 \quad 2006$

$-6138161616$

$\begin{array}{rrr}-113 & 8 & 0 \\ -17 & 30\end{array}$

-16168 o $109 \quad 10516$

$-1614 \quad 8 \quad 136 \quad 12911$

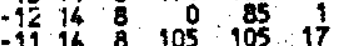

$-10168212211 \quad 7$

894862 83 61

61488216219,13

4148176 155 13

$-314814313910$ $\begin{array}{llll}-3 & & & \\ -2 & 5 & \end{array}$ n 1 lofo lofe 10

$-7158000$

- 1 \%

-1416 81 30

-13 $16800 \quad 1065$

.116080

$-16 \quad 0020$

- 16 ? 35

1780931

111786137 60 s.

1.: 5 कo of 30

3: 6576 ?

3. क क स

6.18 17 in 17

8.189 54033

1.179. $05 \times 10$

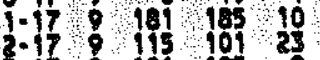

$3.17 \% 191019$

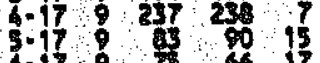

6.179017

$\begin{array}{ll}9.17 & 05 \\ 8.17 & 45\end{array}$

$\begin{array}{rll}9-17 & 0 & 0\end{array}$

$-26,41340$

0.169135

$2=16 \% 17017$ o 17

2-16\% $90 \quad 69$

-169 9767017

5.16? 91101013

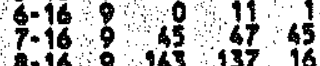

8.16 143716

9.169353

.50109616

- $-3-158 \% 5 \%$

$-2.159191616$

0.150653622

1-15 $950 \quad 147 \quad 10$

3.159162149

6.19960715

$5-15960722$

$7-15 \% 22$ 31 22

$8-15913813908$

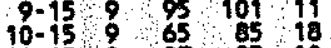

$11-15,97 \quad 9310$

$\begin{array}{lll}-6.16 & 75 & 36 \\ -5.14 & 36 & \end{array}$

$\begin{array}{lll}-4-14 & 9 & 0 \\ -3-16 & 34 & 1\end{array}$

$\begin{array}{lllll}-2-14 & 9 & 197 & 212 & 22\end{array}$

$-1-149 \quad 91 \quad 102 \quad 16$

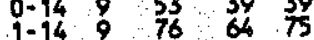

$2-14 \quad 122 \quad 11810$

3149043

$4-149,138,138,11$

$6.149,97,102,17$

$7.149,104,115,12$

$8-149,38,46,38$

10-14 9 102 42 9

$11-149,93,84,8$

$7-13 \% 160 \quad 150,12$

$\begin{array}{ccc}5-13 & 9 \\ -413 & 0 & 196 \\ 5179\end{array}$

$-3139945,14,45$

$\begin{array}{lllll}2-13 & 9 & 241 & 243 & 9 \\ -1-13 & 9 & 52 & 45 & 52\end{array}$
Pase 16

ho l 10\% 10\% 108

0.13 ol

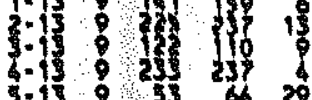

6.15025 of 30

7.151261398

$9.13 \quad 2120 \quad 1018$

$10.15 \% 26 \quad 2015$

$-9.12 \quad 0 \quad 1201120$

$-7.12,91350$

- $12 \%$

-2.12 \& $16017 \%$

0.9283 as 22

$2.7 \%$ o 610018

4.13? 176 161

5.129696016

7.12 . $9 \times 16 \% 1$

$8-12 \% 90100$

$90129110 \%$

$9=2600 \quad 6013$

$12-1290039$

$9.11010 \quad 54$

$-8-11910013215$

$-7.11915916610$

-5.1 919 व

-4.11 9122 117 15

-3119232 233 7

$-1-11916016410$

-11 972169

$2-11015240$

4.1192 28 13

5-11 9176,160

7.1 205105

7-1192206 274

$10-119$ \% 22

$\begin{array}{llll}11-110 & 914 & 118 & 7\end{array}$

$11.10 ? 8077$

$\begin{array}{rll}-10-10,9 & 0 \\ -9-10 & 25\end{array}$

$-8.10 ? \% 10418$

$-7-10,9100 \quad 9720$

$-5-10$ ? 131 133 11

$-4-10$
-3.10

- $-109,178176$

- $1-10$ 9 $237226 \quad 8$

$0-109172 \quad 190$

$1.109127 \quad 1236$

$\begin{array}{lllll}2-10 & 9 & 162 & 168 & 5 \\ 3-10 & 9 & 203 & 209\end{array}$

$4-10.060$

$5-10 \quad 9 \quad 134 \quad 135$

$6-10 \quad 9106 \quad 100 \quad 8$

$\begin{array}{lllll}7-10 & 9 & 287 & 285 & 6 \\ 8.10 & 9 & 157 & 149 & 6\end{array}$

9-10 9 213 213 3

$11-10$, $9127 \times 133$.6

$12-10$, $144,156,6$

$919,965,72,15$

$\begin{array}{lllll}10 & 9 & 9 & 54 & 41 \\ -9 & 53\end{array}$

-8.9. 128,4015

$\begin{array}{llll}-79 & 9 & 197 & 200 \\ -6 & 82\end{array}$

$-5-9.9215216$ 11

- $92915,168,18$

$-20.9138,134,13$

$0.9,9 \quad 129 \quad 139,8$ 
(2)

ratol 11. and celculated structure pector for म) $190010 \mathrm{Fe} 10 \mathrm{~s}$

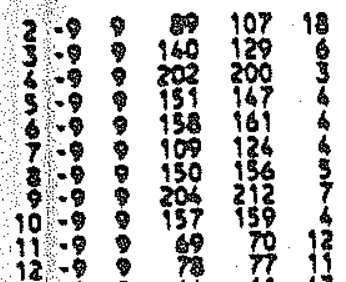

-11:- 6193

-10.8 35 औ

$+7 \cdot 9 \cdot 176,1090$

+6.5. 309276

$4 \cdot 6 \%$

$-3.9 \cdot 13$

1.8 .239229

1. 202200

?.8 $9.216 \quad 210$

4.8. 375

9.5 $9215 \quad 222$

7.8235

10.21825

$11.5 \%$ o 90

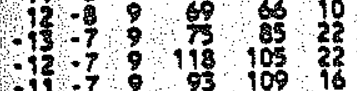

$\begin{array}{ccc}-11.7 & 9 & 0 \\ -10.7 & 0 & 28 \\ -9-7 & 0 & 58\end{array}$

$-8.7 \quad 0 \quad 0$

$\begin{array}{ll}-7 & 255 \\ -5 & 75\end{array}$

$\begin{array}{lll}-5 & -7 & 357 \\ -3 & -7 & 347\end{array}$

$-2-7 \quad 191800$

$\begin{array}{lll}-1 & 03 \\ 0 & 43 & 33\end{array}$

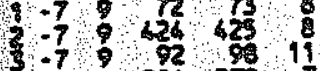

$5.7 \%$. 27211

6.7, 20 272

$\begin{array}{ccc}-7 & 9 & 9\end{array}$

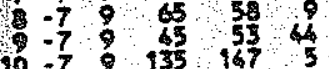

$\begin{array}{llll}10-7 & 135 & 167 & 5 \\ 11-7 & 95 & 81 & 15\end{array}$

\begin{tabular}{llllll}
14 & -6 & 9 & 57 & 50 & 57 \\
13 & -6 & 9 & 173 & 168 & 12 \\
\hline 2 & -6 & 253 & 231 & 7
\end{tabular}

$\begin{array}{llllll}-12 & -6 & 9 & 235 & 231 & 7\end{array}$

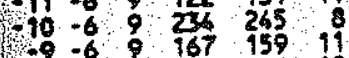

18 $-6 \quad 126126 \quad 12$

$\begin{array}{rrrr}7.6 & 69 & 40 & 4 \\ 6.6 & 9 & 180 & 179\end{array}$

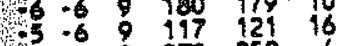

$\begin{array}{lllll}06 & -6 & 9 & 270 & 259 \\ 3 & -6 & 9 & 161 & 134\end{array}$

$\begin{array}{rrrrr}3 & -6 & 9 & 161 & 134 \\ -25 & -6 & 9 & 265 & 252\end{array}$

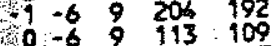

$\begin{array}{lrrr}1-6 & 9 & 110 & 107\end{array}$

$2069,167,154$

$\begin{array}{lll}6.6 & 9 \\ 3.6 & 253 & 242 \\ 213 & 203\end{array}$

96917317510

$\begin{array}{lllll}10 & -6 & 9 & 91 & 77 \\ 1 & -6 & 9 & 65\end{array}$

$\begin{array}{lllll}14 & -5 & 9 & 26 & 42 \\ -13 & -5 & 118 & 140\end{array}$

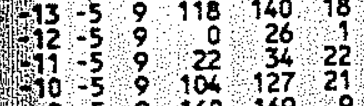

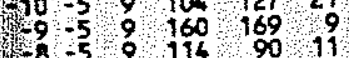

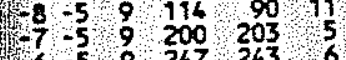

$\begin{array}{llllll}6 & -5 & 9 & 247 & 243 & 6 \\ -5 & -5 & 9 & 293 & 286 & 3\end{array}$

$\begin{array}{lllll}-11 & 119 & 168 & 172\end{array}$

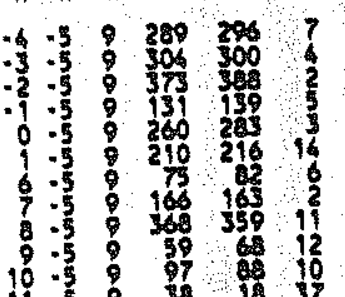

$\begin{array}{rrrrr}10.5 & 9 & 97 & 88 & 10 \\ 11 & -5 & 9 & 38 & 165\end{array}$

-16 -6 60 \% 16

$-13.6 \% 1627611$

110.49240235

$4.4 \% 21821319$

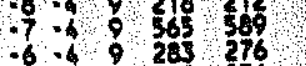

$\begin{array}{lll}-6 & -4 & 253 \\ -5 & -4 & 276 \\ -5 & -4 & 236\end{array}$

-4. $6 \quad 292$ 290

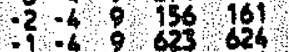

0.49623401

$6.49,237,234$

8.912613010

10.9 3 65 37 12

11 .6 113105

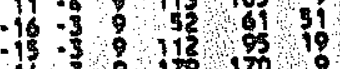

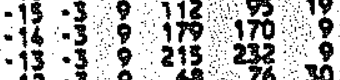

-12.3 9657630

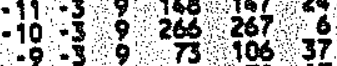

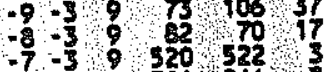

$-7-39501225$

$-3-396967$

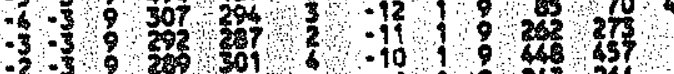

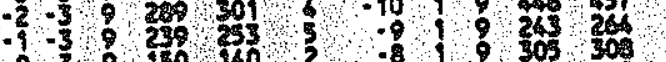

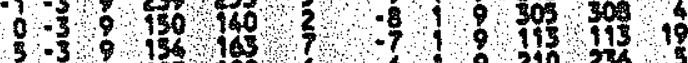

- -3.915150

7.392272013

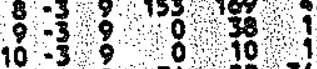

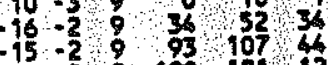

$-16=2,9001918$

$\begin{array}{lll}12 & -2 & 220 \\ -212 & 11\end{array}$

$-10-2,9967026$

$\begin{array}{lllll}-9 & 2 & 9 & 225 \\ -8 & -2 & 9 & 369\end{array}$

$-8-2,956{ }^{369}$

19103111 10

$-6=2 \quad 812819 \quad 13 \quad-18 \quad 2 \quad 128,11929$

$\begin{array}{llllllllllllll}-4 & -2 & 9 & 78 & 76 & 10 & -17 & 2 & 9 & 134 & 136 & 13\end{array}$

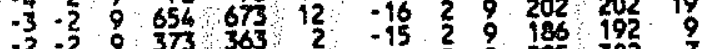

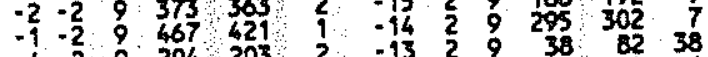

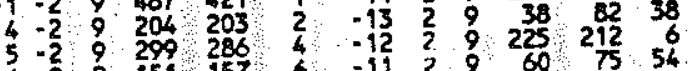

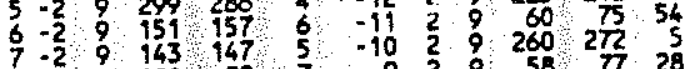

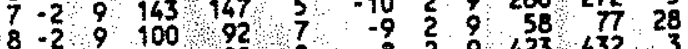

$9-2,998,98$. 8

$1902,90171 \% 178,11$

$17,99,81,72,42$

$-15, ? 966,65,66$

\begin{tabular}{rrrrr}
14 & 9 & 109 & 115 & 24 \\
\hline & 96 & 20
\end{tabular}

$\begin{array}{rrr}-12 & -199 & 94 \\ -12 & 945\end{array} 9$

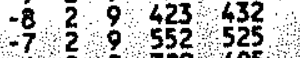

62,339405

42,9609615

$\begin{array}{lllll}-3 & 2 & 968 & 432 \\ -1 & 242 & 237\end{array}$

-129344356 ?

$0 \quad 2 \quad 9 \quad 138 \quad 126 \quad 13$

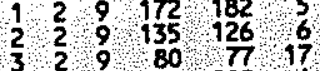

$8.19,15414799$

$-7.19,531,208,10$

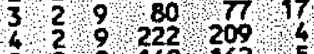

$529,160,162$, 5

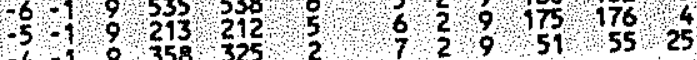

h $k$ i 10Fo 10F 100

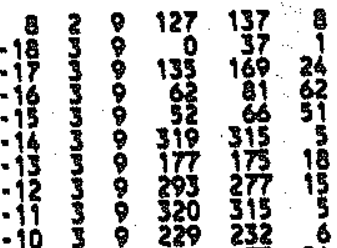

-10 90229232

- 7 \$ 9817 277

-639 \% 50

\section{- 360 258}

13940317

138169 , 2215

$3 \quad 9 \quad 1681720$

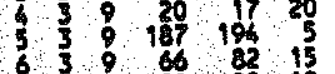

$639.66 \%$

$186 ?$ ? 175,160 ,

$16 \% 8$ - 159 138 13

- I1 01727

$-12 \quad 270$

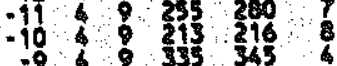

-9 $9 \quad 121$ 119

$-7 \quad 126$
$-5 \quad 220$

- 62,210025

$\begin{array}{ll}-2 & 90 \\ -1 & 106\end{array}$

$0 \quad 353^{30} 3$

$3 \quad 171919$ ?

5210100

6.9 .583016

$-1859037$

$\begin{array}{lll}-17 & 5 & 153 \\ -16 & 56 & 11 \\ & \end{array}$

$\begin{array}{rrrr}-15 & 5 & 217 & \\ -16 & 5 & 238 & \\ -13 & 5 & 0 & 0\end{array}$

$\begin{array}{llll}-13 & 5 & 0 & 48\end{array}$

$\begin{array}{lllll}-12 & 5 & 0 & 86 & 92 \\ -11 & 5 & 327 & 324 & 4\end{array}$

- $-959125185 \quad 9$

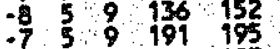

$\begin{array}{llll}-6 & 5 & 964 & 183 \\ -5 & & 220 & 212\end{array}$

$\begin{array}{llll}-5 & 5 & 9 & 220 \\ -4 & 5 & 212 \\ -3 & 5 & 225\end{array}$

- 3 . 5 \% 302 299

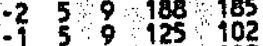

$\begin{array}{lllll}0 & 5 & 9 & 187 \\ 1 & 5 & 9 & 315 & 340\end{array}$

$\begin{array}{llllll}2 & 5 & 9 & 130 & 127\end{array}$

35913914011

5 5 570 . 58,20

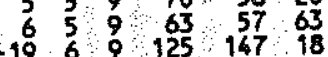

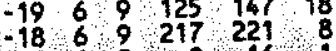

$-176690125,121,13$

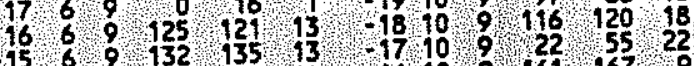

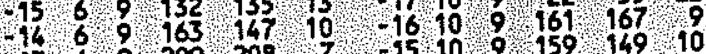

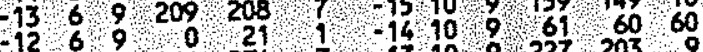

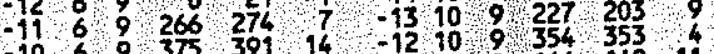

$-1069,375,175,8,-1110$, $, 144,142,11$

$8.69242,234,19$

7 6.9. 360 , 343,4

${ }^{10} 10.9,242,233,8$

$810,9.0 \% 12$ 1

\begin{tabular}{lllllllllll}
5 & 699 & 102 & 190 & 10 & -7 & 10 & 63 & 50 & 31 \\
\hline
\end{tabular} o 10

2253225

69121 . 11

1979.102125

167909

27860

$979106 \% 2$

$\begin{array}{lll}7 & 7 & 905 \\ 6 & 7 & 90\end{array}$

2933306

1796563 ?

7. 91 13 40

-17 8108150

918

$-98916216130$

68917519

-3 94464

8. 9228224 ?

$\begin{array}{llll}1 & 8 & 178 & 198\end{array}$

$1909.227220 \quad 7$

$1709173 \quad 15326$

$\begin{array}{llll}15.960 & 352 & 12 \\ 80 & 25\end{array}$

-12 922522626

$1092168166 \quad 8$

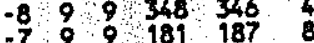

$\begin{array}{llllll}-6 & 9 & 9 & 371 & 368 & 6\end{array}$

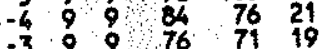

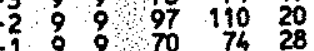

$09 \quad 9197 \quad 196 \quad 8$

299110 121 15

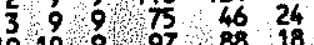

47228265

- $\quad 9100$ 109 11 
T61. 11. onom calculated structure factoru for 1

1. I $1070.108 \mathrm{c} 100$

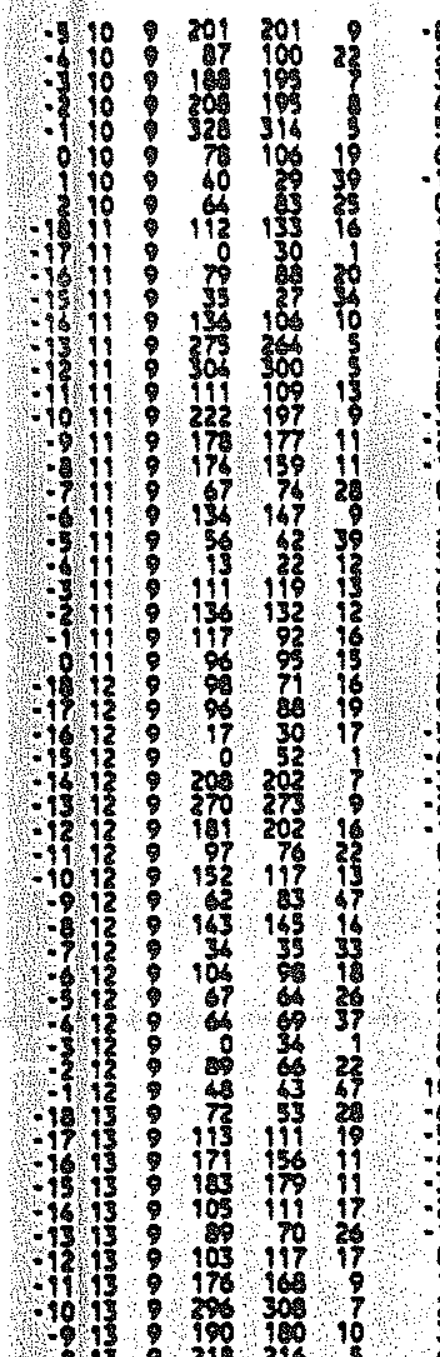

$-913.218216$

$-19196190$

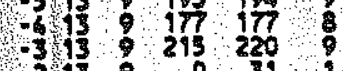

$\begin{array}{llll}-1713 & 0 & 0 & 31 \\ 102 & 15\end{array}$

161690000

16139202020

+1216 92313818

1016999 168 12

Q16 $9176 \quad 180 \quad 9$

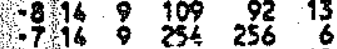

$\begin{array}{rrrrr}0614 & 9 & 227 & 219 & 12\end{array}$

$\begin{array}{rlll}-6145 & 138 & 146 \quad 14\end{array}$

$\begin{array}{lll}-15 & 9 & 42\end{array} 49$

$46599,116 \quad 116,15$

-12 15 9 ? $222,101,17$

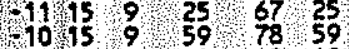

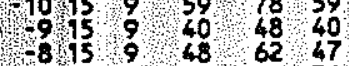

$\begin{array}{rrr}815 & 48 & 62 \\ 4 & 47\end{array}$

-615\%, 88 911719

$41516,9,126,117$, 17

13 $16 \% 9,0$ \% 19

$1116,9 \% 66,46,31$ h l 10po pope tos

3.1 10 r 70 \%

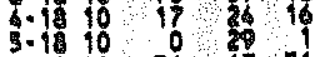

-1910 35 67 \%

0.1710 30 13

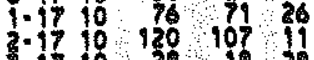

s.17 10 28 10 103

3.1710 ह

9.1790 .76 का का

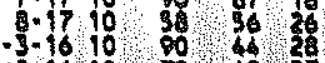

$-2.610 \% 247$

0.1610 \% 109 .16

1.1610 30 3529

$3-1610 \quad 46 \% 5$

-16. 10 \%

3.1610 \% 76 16

6.16 10 19. $91 \% 17$

9.1610014136016

$-5191000 \%$

- -1510 122 12310

0.1510 119 2611

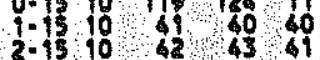

2.15 10 62 231

- $1510 \quad 105$ 10 27

3.15 10 70 का

7.1510075017

$10.9510 \quad 113$ 26 15

- $1610 \quad 63743$

- -1610 132 1511

-3.14 10 19 19

$\begin{array}{lll}-2-16 & 10 & 134 \\ -1 & 16 & 0\end{array}$

0.16 10 g 8939

2. 14019128

3.16 10 क 50

S-16 10 क 55

$6-1610,112,105$

7.160109015

$\begin{array}{lll}8.16 & 10 & 133 \\ 0.14 & 130 & 9\end{array}$

$10-1490$ 105 100415

$-7.131011614219$

-6.1310 13. 190,14

- -1310 16 3428

$-3-1310 \quad 60 \quad 6931$

-2.1390 134 1539

0.1310 18 188

1.15 10 122 111 18

$=1310 \quad 112$ 95 16

$3.130170 \quad 17510$

$\begin{array}{lllll}6-13 & 10 & 77 & 59 & 16 \\ 5-13 & 10 & 0\end{array}$

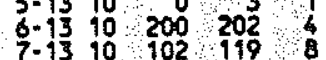

$8-1310,148,150,10$

10.1310 .112 193 ?

9.1310 . 80,87 . 8

$\begin{array}{r}-12 \\ -70\end{array} 85,7868$

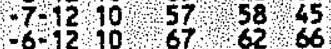

$-6 \cdot 12,10,67,16266$

$-4-92,10,15,152,14$

-11210 98 11320

0.12104937916

$\begin{array}{llllll}1-12 & 10 & 19 & 102 & 9 \\ 2 & 12 & 10 & 102 & 69 & 12 \\ 3-12 & 10 & 124 & 123 & 12\end{array}$
(16 1089746

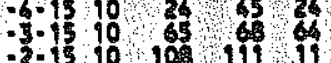

$-2-12.100125$ र 1112 h $k$ I lopo lope 10s

4.12 10153155

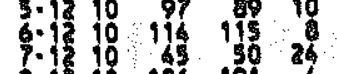

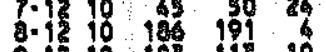

10.1210 105 115 is

11.1210 \& 525

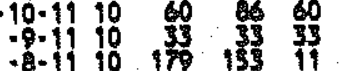

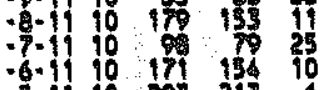

- -1110 20 1013

\begin{tabular}{c|cc}
-3.1 & 10 & 236 \\
-2 & 261
\end{tabular}

10110 13 $160 \%$

4110 19 $100 \%$

3.1110 15 193

5.11 10 का 100111

$7.1110,107,120$

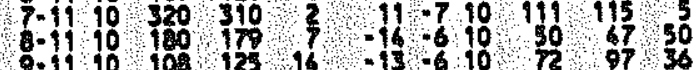

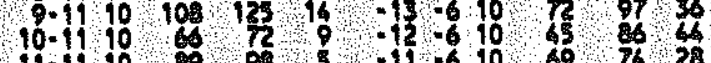

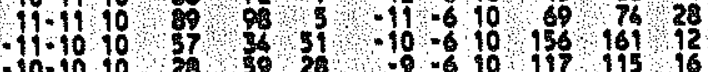

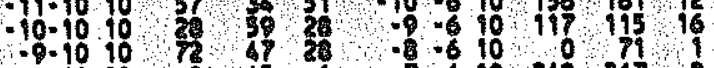

-7.10 10 o2 1013

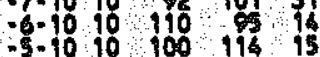

- $-101015 \mathrm{~s} 15 \mathrm{~s}$

- -10 i 250207

- $=10.10,16527$

$0.101051 \% 51$

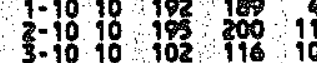

3.10 10 10 10

$5-101011512$

$6.10 \%$ o 37

7.10 10 , 152

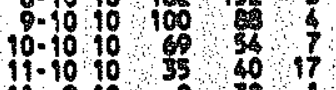

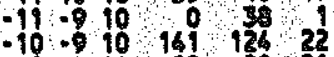

- 890 . 90 ह 7021

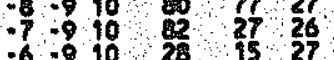

$\begin{array}{llllll}-6 & 9 & 10 & 28 & 15 & 27 \\ -5 & -9 & 10 & 133 & 138 & 13\end{array}$

$\begin{array}{llll}-6 & -9 & 10 & 0 \\ -3 & -9 & 02 & 45\end{array}$

$\begin{array}{lllll}-3 & -9 & 10 & 229 & 222 \\ -2 & -9 & 10 & 153 & 158\end{array}$

$-1-910$ lo 20010

$\begin{array}{llll}0.9 & 10 & 117 & 121 \\ -0 & 10 & 130 & 125\end{array}$

2910 द66 139

$3.910 \quad 157 \quad 161$

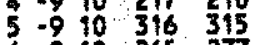

$6-910 \quad 265 \quad 277$

$7.910 \quad 166 \quad 156$

$8 \quad 910 \quad 267 \quad 270$

9.9 90 137 139

$19-919 \quad 97 \quad 102 \quad 6$

$\begin{array}{llllll}-12 & -8 & 10 & 41 & 72 & 49 \\ -11 & -8 & 10 & 93 & 89 & 18\end{array}$

$-10-810 \%$ \% 18

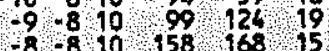

$\begin{array}{rrrr}7 & -8 & 10 & 56\end{array} 30 \quad 55$

6. 10 108 12420

$-4-810 \quad 218 \quad 219.5$

$\begin{array}{lllll}-3 & 8 & 10 & 39 & 66\end{array} 39$

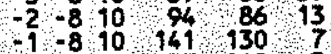

$0.810 \quad 157 \quad 149$ क

$\begin{array}{lllll}1 & -8 & 10 & 239 & 228 \\ 2 & -8 & 262 & 269\end{array}$

$\begin{array}{llllll}2 & 8 & 10 & 262 & 269 \\ 3 & -8 & 10 & 411 & 391\end{array}$

$-7.610 \quad 269267$

-6
-5

- $-610 \quad 172$ 171

$\begin{array}{llll}-2 & -6 & 0 & 257 \\ -1 & -6 & 0 & 345\end{array}$

0.810199193

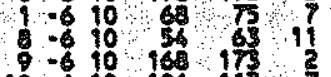

$10-610$ 106 173

$-16-510 \quad 70 \quad 39 \quad 28$

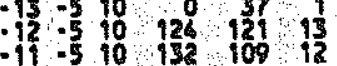

$-10=50115$ 14

-9 -5 10 108 16812

$7-51015016719$

$-6.510 \quad 62600$

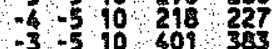

$\begin{array}{llll}-2 & -5 & 10 & 160 \\ -166 & -5 & 10\end{array}$

$0-510$ 417 63

$8-510 \quad 98109$

$10-510 \quad 170-37$

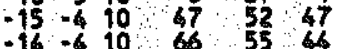

$\begin{array}{llll}-14-610 & 66 & 55 \quad 68\end{array}$

$-12-610$ o 9818

$\begin{array}{rrrrr}-11 & -4 & 10 & 56 & 71 \\ -10 & -610 & 179 & 169\end{array}$

$-9.410 \quad 194181 ?$

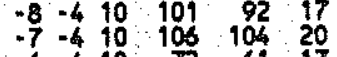

$-6-410 \quad 72 \quad 61 \quad 17$

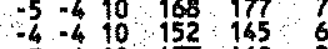

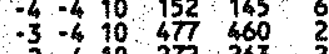

$\begin{array}{lllll}* 3 & -4 & 10 & 272 & 263 \\ -1 & -4 & 10 & 291 & 301\end{array}$

$\begin{array}{llll}-1 & -4 & 10 & 29 \\ 0 & -4 & 10 & 301\end{array}$

$\begin{array}{lllllll}7 & 410 & 18 & 69 & 7\end{array}$

$9,610 \quad 0,20 \%$

$\begin{array}{ccccc}10 & 4 & 10 & 0 & 12 \\ 0 & 63\end{array}$

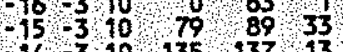

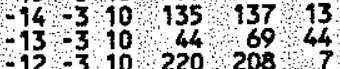

$\begin{array}{rrrrr}-12 & -3 & 10 & 220 & 208 \\ -11 & 3 & 213 & 210 & 7\end{array}$

$10.310 \quad 234,232,6$

- 310 116 131 12

$\begin{array}{rllllll}8 & -3 & 10 & 193 & 181 & 6\end{array}$

$\begin{gathered}48 \\ 5\end{gathered}-810,447,435$
Pog 18

n. 11060 bofe 108

-5 - 310 of 100

- 10 - 15

- -310421003

7.510 व

8 - 10 13 2013

$10.10 \quad 76$ \% 11

-15 -z $10.36 \quad 12$

$-14=210123106$ is

-13 -2 $10.157,166.13$

-10.310 20 27 22

-9.21010171 .

-7.210 10101910

$-5-210118120$

- - 1019200

- -2 -2010426 422 10

-2 10 a1 8

6.210 31 10 30

$\begin{array}{ccc}7-2 & 10 & 97 \\ 9-2 & 2030 & 7\end{array}$

$17-110,50 \quad 56 \quad 31$

$-16-10 \backslash 51$ 55

$-13-1000$ a 22

- 130 - 207177 ?

-110 I21 11611

- 10.10 - 160 का 34

$-7-110239$ 219

- $-110 \mathrm{k} 1242$

- -10 255 25

$-2-110$ z3 277

$0.110262=30$

1.1032720

$2=10146140$

3.110255255

- 10165151

$6.110 \% 908$

$7-10,32 \quad 5931$

$8-110 \quad 27027$

$\begin{array}{llllll}-17 & 0 & 10 & 0 & 55 & 1 \\ -16 & 0 & 10 & 15 & 56 & 33\end{array}$

- 15010150,14811

- $14010 \quad 70$ \% 175

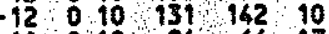

$\begin{array}{lll}-11 & 0 & 10\end{array} \%$

- 01012611610

-8 $010 \quad 274 \quad 259$ i3

$-70101761876$

$\begin{array}{lllll}-6 & 0 & 10 & 321 & 337\end{array}$

$\begin{array}{llll}-5 & 0 & 10 & 333 \\ -4 & 0 & 30 & 8\end{array}$

$\begin{array}{llllll}-3 & 0 & 10 & 329 & 341\end{array}$

-2 01064655

- $1010 \quad 325319$

01032,309

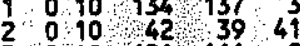

4. 010 121 $185111 \% 8$

$\begin{array}{llllll}5 & 0 & 10 & 176 & 181 & 7 \\ 6 & 0 & 10 & 231 & 218 & 8\end{array}$

$\begin{array}{llllll}7 & 0 & 10 & 23 & 218 & 77\end{array}$

$\begin{array}{rllll}8 & 10 & 39 & 56 & 31 \\ & 17 & 43 & 21\end{array}$

16,10 , 97 107 23

-14110 24, 249 , ?

$-121102246248$

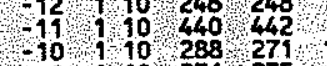

$\begin{array}{llll}-10 & 110 & 288 & 271 \\ -9 & 236 & 235\end{array}$ 


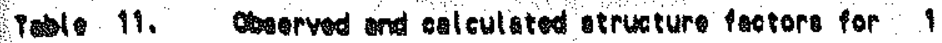

P. 19

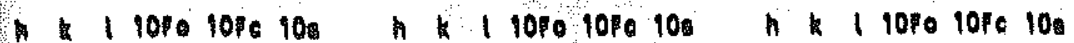

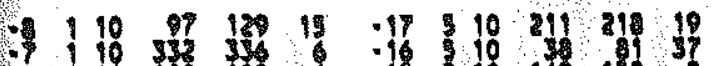

.10 is 109

- 10 需

$-110179$

- 190 is 105 is

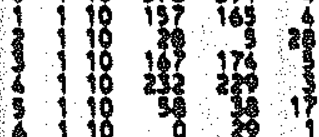

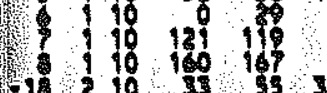

19. 18 , 137,135

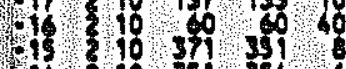

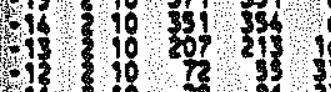

19,10 a 630

H 10 ? 103

T.

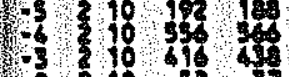

-1 110 2 27

$0.10 \% 10$

13 10 10 1019

18 10 10 101

2. 10 131 155

18 10 10 917

4131027321

13 110 का 100

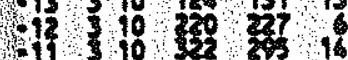

10 10 10 ox

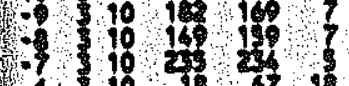

- -110 214 z32 1

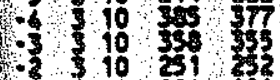

- 10 10 $1977^{5}$

- 10 19 1973

$1310 \quad 2 \quad 76$

$1310 \quad 260 \quad 236042$

19.10 14 1112

$16 \$ 10 \quad 10 \%$ 165 20

$16 \quad 102032068$

$13 / 10$ 13 120010

11 $10,120 \quad 121 \quad 22$

1910 85 3514

$\begin{array}{llll}7 & 610 & 23 \\ 7 & 10 & 238 & 250 \\ 371 & 379\end{array}$

$44 \begin{array}{llll}6 & 10 & 37 \\ 4 & 205 & 34 \\ 34 & 31\end{array}$

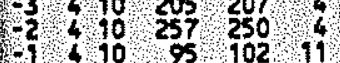

$0,610245264,2$

2. 410 27, 9715 . 11

$34100,76, \frac{5}{53}, 16$

6 $610127,146 \%$

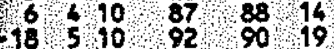

-1 10 1615

- 5101010

-10 10 100 16

- 10 . 10 of 10

. 10 \% $40 \%$

- 10 10 1010

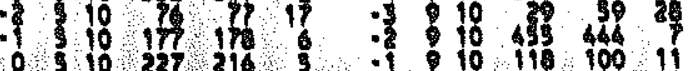
$3 \% 10 \%$

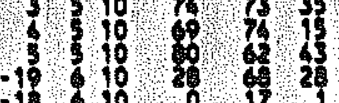

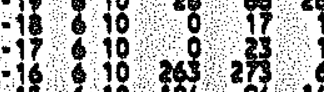

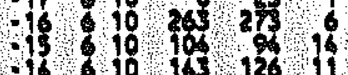

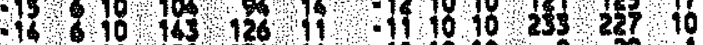

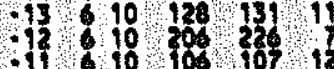

$-10 \% 1019671$

- 110,1613

.61016140

-3 1010 \%

- 110101010

-1.10 $10 \%$

26.03030

$19610 \quad 0 \quad 10019$

$.19710 \mathrm{0} / 50$

.17710 19 16313

- $157 \% 171953$

- 1710 का 217

-10710 रु 267

- 710 253 291

.7710 \% 82

-5 710 198 2036

- $3710 \quad 183 \quad 2006$

$\begin{array}{lll}-2 & 710 & 201 \\ -1 & 204 & 16\end{array}$

-1710
07
1

$171065 \% 9017$

$3710 \quad 32,2231$

$-19 \quad 8 \quad 0 \quad 0840$

$.17810 \quad 177160 \quad 29$

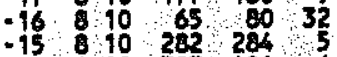

- 1510 $393 \quad 0046$

$\begin{array}{llll}-13 & 8 & 126 & 132 \\ -16 & 8 & 16\end{array}$

$\begin{array}{lllll}-12 & 8 & 10 & 133 & 133 \\ -11 & 8 & 102 & 102 & 91\end{array}$

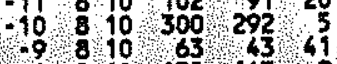

-8810 135 14359

$\begin{array}{lllll}-7 & 8 & 10 & 71 & 35 \\ -5 & 8 & 10 & 267 & 200\end{array}$

$\begin{array}{rlllll}-5 & 810 & 218 & 200 & 8 \\ -3 & 10 & 96 & 304 & 13\end{array}$

\begin{tabular}{llllll}
-3 & 8 & 10 & 100 & 394 & 3 \\
\hline & 8 & 230 & 237 & 6
\end{tabular}

1 8 10 , 429,425 ,

$18810,209,234,6$

? $8100,143 \quad 441410$

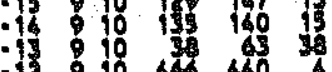
-11 900 105

-9010 130 131 - 91037 . - 910 is $12 \mathrm{a}$ is - 10 st 5 . 9101951 $.151010,16 \%$ का . 161010 \% $10 \% 10$ is 1010 211 213 161012013617 $=1010$ 217 215 6 .71010 I5 1601 - 1010,1618 -21010 16 IS 91010 (1) -18 110 s 50 -1611 10 10 107 -15 10 130 13 12 13110 167 10

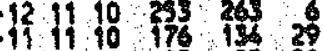
101110 \% 250 - $110 \% 3$ - 110 Iा 1 -31110 os 75 - 1110 6 62 36 -181210 69 616 -16 $1210 \quad 126 \quad 102$

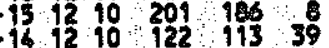
- 13121017017612 $\begin{array}{lllll}-12 & 12 & 227 & 215\end{array}$ 101210 130 $107 \quad 19$

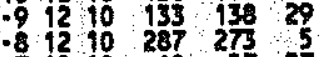
$-71210 \quad 60 \quad 57 \quad 27$

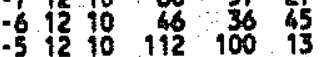
$\begin{array}{llllll}-4 & 12 & 10 & 0 & 46 & 1 \\ -3 & 12 & 10 & 190 & 195 & 8\end{array}$

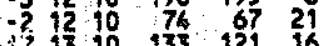
16131011909 $-161310 \quad 150 \quad 104,14$ $131310,71,78,28$ 111310,89 , 93,19 -91310 64 86,63 $81310,77,7725$ .61310213228 ?

$-51310 \% 898618$ $-31310,43.57,43$ $-171410 \quad 6305638$
$-18910 \quad 401740 \quad-141510$ ol 1029

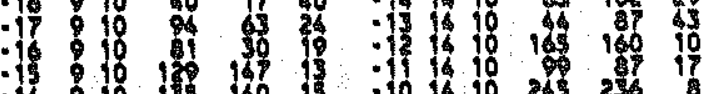
- 10 ig 100 -71110 11 11 $161410, \frac{45}{32}, 26,44$
- 140 17\% 155 - 1610 in 12 - 61610 \% 115 2 - 10 : 10

15151000

-1110 is 1150

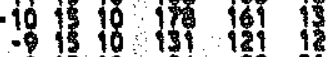

- 1510 क 96 1161606070

o:-17 11 1360 126 12

2:-17 1 27 43 27

\&.7 11 0 \&

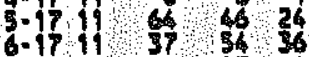

-3. 1611 0 27

$-1.16115535$

0.11 क 104

$1-1611079$

3.16 19001

5.16 II 197 16

6.1619 89651

.16 11 0 1

- +5.1511060

- -15111914010

1.15111605

0.1511165135

$2+1511070$

3.15117472

S.15 11 11 105

\%. 11 का

7.1 11 का 5

9. 31100

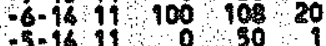

$-5.1411 \quad 0 \quad 50$ क

$-4-141137 \quad 4237$

-2.16 $11 \quad 202 \quad 300$

$-1.16111721746$

$0-1411 \quad 92 \quad 83 \quad 12$

3-1411 87 64 14

5.14 11 61 $55 \quad 16$

$6.911 \quad 87 \quad 95 \quad 11$

$7.1611 \quad \frac{56}{5} \quad 13$

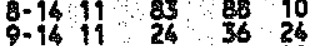

$-7+1311101032$

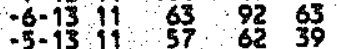

$-4-1311 \quad 115 \quad 11512$

-2-13 $11 \quad 50 \quad 3549$

$\begin{array}{lllll}-9-13 & 11 \quad 131 & 126\end{array}$

0.131606520

$2-1311879192,6$

$4-1319$ 1 126 , 81 , 12

$5-131\} \quad 64,45,15$

6.13.11 82 - 73 \% 10

8-13 11 61 28 13

$9-13,11,22,13,22$

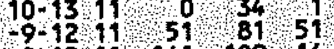

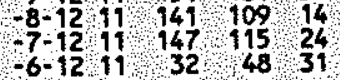

2-14 11 157 14510 h l toro lofe 100

-511111 \&

-13 1 \&

1.12 ओ 1110 21

1.121109 19

अ.12 1 23 23 3

5.12 110 of 10

6.12 11 1350

8.1211117 125 6

$10.1211 \%$ क

10.11116025

- -1111100 हू 20

: 8111100 \& 20

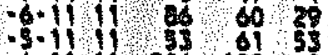

- $3-1111200$ 192

-2.11 11 os

- -1111 is 87 is

1.111105 5 50

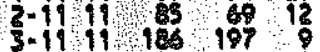

4.11 11 121 ? ?

$6-1112231227$

7.1111 का 112

9.1111096

$.19-1011003$

-10.1011377 75 39

8.1011021320

-7.1019115 in 15

-5.10 11 40 24 40

-4.10 11 $170 \quad 180$

- 1011118 123 10

1.10 11 14213510

$1-1011939142$

2.1011 192

- 1019200293

5.10 1119103

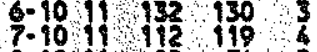

8.10 1 65 745

9.1011 31 $\quad 32 \quad 20$

-11.91157 \& 49

$-10.911 \quad 68 \quad 55 \quad 35$

-8 -919960016

$-7.911172168$

$-5-91114213611$

-6 -919, 69 56 56

-2.911 t5 70 15

$\begin{array}{lllll}-1 & -9 & 1 & 120 & 130 \quad 7\end{array}$

$0-911177$ in

$2-91125 \quad 81 \quad 12$

$3-911 \quad 131 \quad 130 \quad 8$

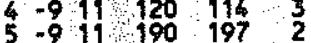

$\begin{array}{lllll}-12 & -811 & 76 & 71\end{array}$

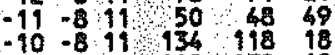

-9 $-811 \quad 182$ 178 19

$\begin{array}{rrrr}-8 & 811 & 65 & 44 \\ -7 & 80\end{array}$

$-6-811111670$ 21

$-5,811,212,215,6$

$-381,29910215$

$-2811213210,6$

0.811934135 5

$1 - 8 1 1 \longdiv { 5 9 } 5 4,17$

2.81. 160 \% 8

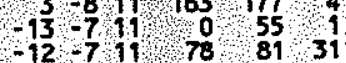




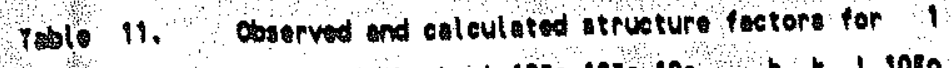

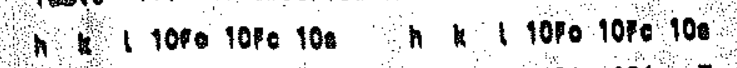

$11-71190091$

$-71110014 \%$

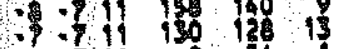

- 711 ? 3

$* 4.711909$

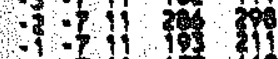

0.7116

2.71010

$0 \% 611$ क 6

$.11 \% 11$ क 50

$0 \% 11456129$

9611233

68111219

$641190 \quad 1018$

$-361127 \quad 20127$

-1 11 क 60 ?

1061 क 35

11311574

-1951 1510

-9 11 10 19 ?

$0.9411,160$ ? 16

-6 I1 207 3)

-351119 19

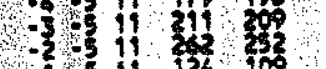

-195 124 109

95 \% 71,16

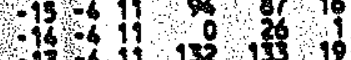

13 11 110 120

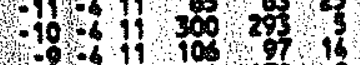

-0.611 147?

- 7.6 111309 150

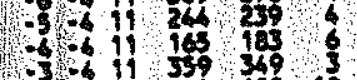

-3.6 11 162 132 10

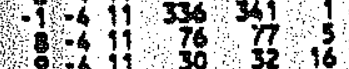

16. $11{ }^{30} \quad 3216$

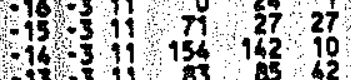

$-13.311 \quad 210 \quad 206 \quad 7$

$\begin{array}{lllll}211 & -3 & 258 & 263 & 6 \\ -10-3 & 301 & 316 & 7\end{array}$

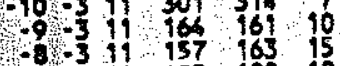

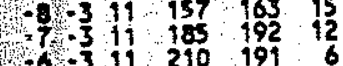

$\begin{array}{lllll}-6 \cdot 3 & 11 & 210 & 191 & 6 \\ -3 & -3 & 116 & 20 & 9\end{array}$

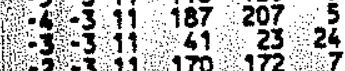

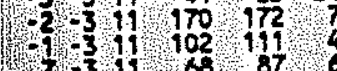

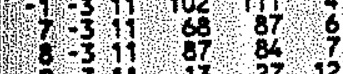

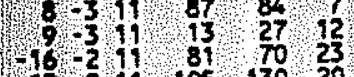

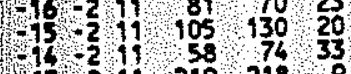

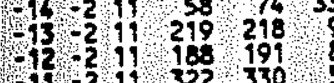

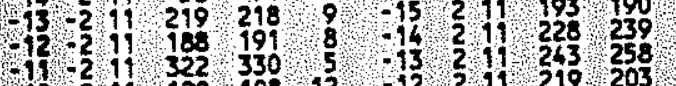

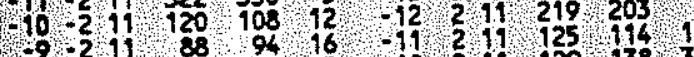

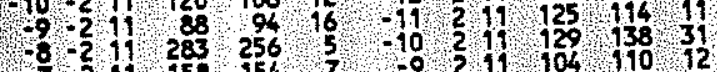

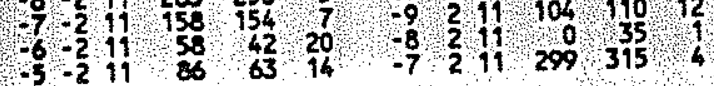

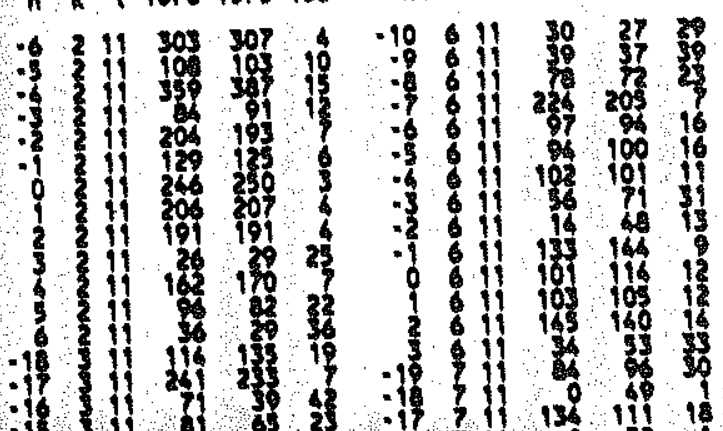

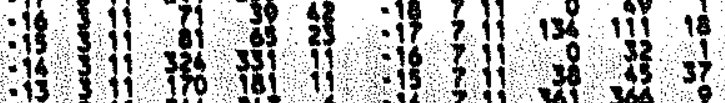

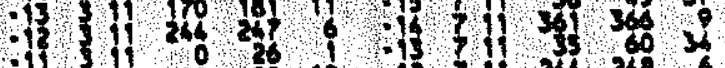

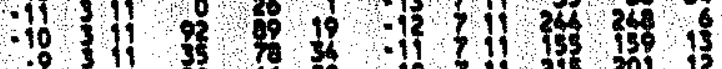
$\begin{array}{lll}-0 & 3 & 3 \\ -7 & 53\end{array}$ $* 3110373510$ .3311626450 ? 43116036

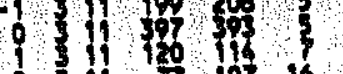
$311010 \%$ $3113011 \%$ .111551672 $.1611100 \%$ .1519 1403 - $1311 \%$ $.11 \% 11$ 1 13162 61117617 का -7 I 373 $-61112$

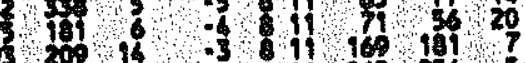

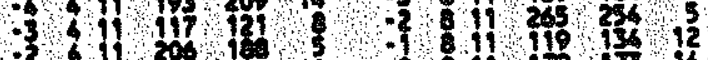

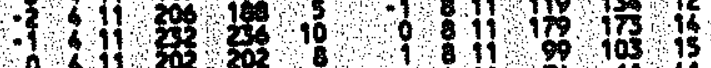

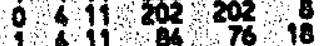
$1131020 \%$ 311121 1का 3 31136 $-18,1106 \%$ $-165112162231$ -15
-16
-13 $-13.51910319010$ $-115110220230$ -10 11 222 150 $\begin{array}{llll}-9 & 5 & 1106 & 06 \\ -7 & 15 & 10\end{array}$ $-75111101181$ $\begin{array}{lllll}-6 & 5 & 108 & 111 & 11 \\ -5 & 5 & 240 & 251 & 52\end{array}$

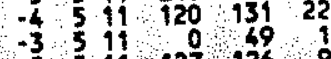

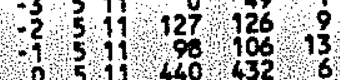
(1) 112214 203 25

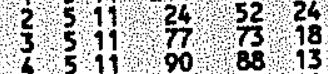
$-19411 \% 65 \% 36,49$ .18611 21 6921 $-16611,121,130,17$ $-15611,121$, 93 17 01461199,14917

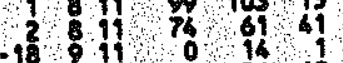

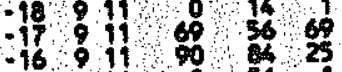
$\begin{array}{lll}-16 & 9 & 90 \\ -15 & 9 & \end{array}$ - 14 9 11 20 20013 :12. 911901928 $-11911 \quad 85$ \% 20 -10. 91130 133 11 8911 154 164 . 6 - $911102 \quad 8812$ $-5911 \quad 14113412$ - 39112812612 -2911 141 138 19

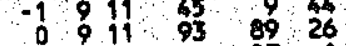
1911002701
-18101101530 171011 47 18,66 $151011,16146,3$ -131011 126 2836 ? 27 $121011 \quad 233$ ?227 10 $-101011{ }^{178} 5454510$ $91011129,133,10$ $-71011 \quad 84,8421$ $\begin{array}{rrrrr}6 & 10 & 11 & 168 & 154 \\ 5 & 10 & 243 & 239 & 7\end{array}$

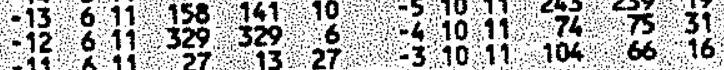

Page 20

- I lifo lofe 100 -2 1011 19 or 14 16 if 1940 if 11 की 67 \% 12 11 107102 11 II 118100 - 119 in 150 $-711115116112$ 711137162 35 - If 101100 3111130 के $17121167,100 \%$ $1512110 \% 100$ $1611330 \% 1$ .121123925012 .111211962162 - 11150153 012111803103 71211163144 -5 2116496 - 1211 की 38 -1713 11 का 1837 - 16 is is - 14 11 130 141 - 111311 170 100 12 - 91311260250 -9 911100 का 615116926 $-1615114303$ -15 161155067 -13 1117221073 $.1161106,14211$ - 016111231231 $-6161106275$ $-15110 \% 21$ $-131510061$ -11 11192216 $101712,38,68$ $2-1712$, 77 , 71 $6-171249848$

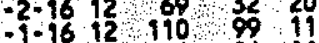
$-1.1612108016$ $1-16 \quad 12 \quad 51$ 26 51 $\begin{array}{lllll}2-16 & 12 & 102 & 79 & 14 \\ 3-16 & 2 & 52 & 52 & 40\end{array}$ $\begin{array}{lll}3-16 & 12 \\ 52 & 52\end{array} \frac{52}{29} \quad 40$ $\begin{array}{lll}4-16 & 12 & 132 \\ 50 & 82 & 16\end{array}$ $6-1612 \div 6458$

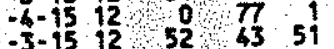
$\begin{array}{lll}-2-15 & 12 & 89 \\ -1 & 16\end{array}$ $0.1512,0,131$ $2-512,27,34,26$ $\begin{array}{lllll}4-15 & 12 & 165 & 138 & 9\end{array}$ $\begin{array}{lllll}5.15 & 2 & 67 & 69 & 21\end{array}$ $\begin{array}{lllll}6-15 & 12 \\ -15 & 85 & 80 & 80\end{array}$ $-6-1412,20,37,19$ $\begin{array}{lllll}-5-1412 & 0 & 40 & 1 \\ -4 & 38 & 38 & 38\end{array}$ $-4914 \quad 12 \quad 38$, 138,18 $21412,80 \quad 61 \% 19$

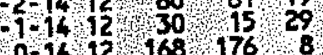




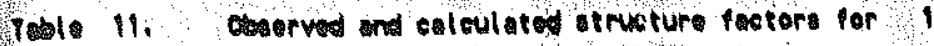

Paro 21

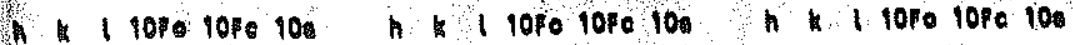

1.14 30 30

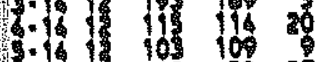

\% 113

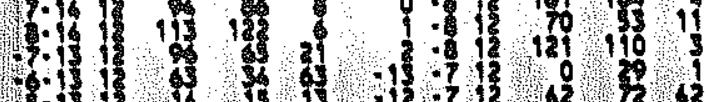

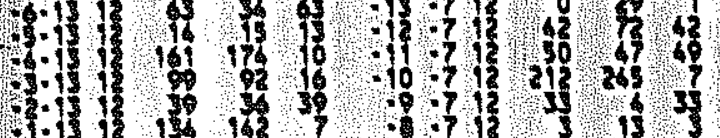

0.1 1916

$1.13 \%$

I 1 10

y.I 1215112

\%.1 15 14 15

-

\%.1 0 og

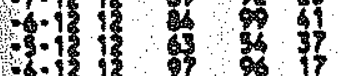

\%16 o 1

\%.1 \%

0.1110015

$\begin{array}{ll}1.1 & 10 \\ 6 & 10\end{array}$

5.12 1392

6.1 1 11

\%. 11101

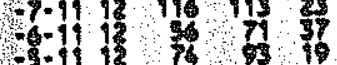

**11 1121112

o.

- $-1127 \%$

5-11 19 10

10.101231

-9.10 1 161672

710 15 1429

- $\% 1010$ is 137

- $3.1012 \quad 12003$

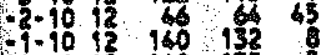

0.10 iz 140135

$\begin{array}{lll}1.10 & 12 & 90 \\ 200 & 00\end{array}$

6.10 i2 1251049

41.912 92 9647

8.91260 .3846

7.91211310819

6.912018 ?

-5.912 162 124 25

$4.912120120 \%$

4.912164 167 11

$0.912,125,111 \%$

1912166175

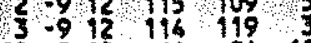

$-12.812 \quad 61 \otimes 60$

$41=812 \quad 49 \quad 43 \quad 41$

$\begin{array}{rrrrr}-10 & -8 & 12 & 8 & 81 \\ -9 & -8 & 12 & 278 & 279\end{array}$

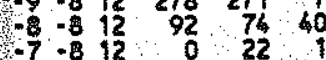

. *

- -12 16 10

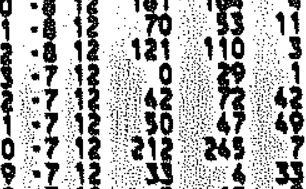

$7 \% 165132$

67165151 ?

$.73 \log 10$

$.7,7151615710$

$0,7,3516 \%$

.14 .61 \%

$-13.403028$

-11 - 16 107 107 1

$-10.612 \quad 160$ int?

$.01 \%$ का

- .612 21519

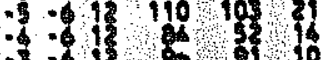

- .6 15 1510

- . - 1 1 151

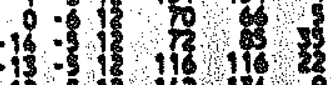

- -515 काष 10

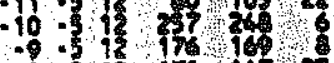

$-7.51701672$

- - 51209101

- -311110

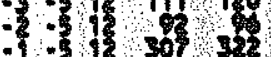

$13-5125074$

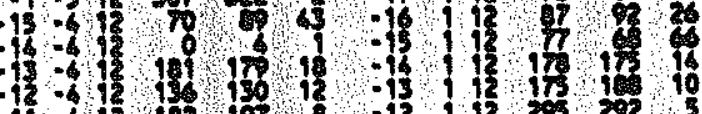

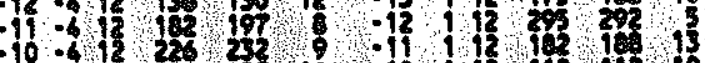

$0.612159+1319$

$-7.612730$

$-6.61217019$

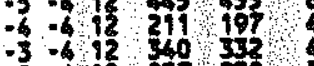

$\begin{array}{llll}-3 & -6 & 12 & 30 \\ -2 & -6 & 237 & 330\end{array}$

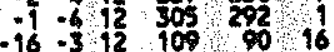

$-16-312109 \% 0010$

$-16,312,160$, 138,38

$12312,257254.6$

$-110.312,267.244$. 8

$\begin{array}{llllll}8 & 312 & 156 & 160 & 10\end{array}$

$-7-312,26222312$

$-6.312,1851296$

$-4-312$ (59 69 17

2 ? 12 126 121 .

$96-212, \quad 32,4931$

$-15-213,107$. 8719

$-13-21213 \quad 1022$

$\begin{array}{llll}-12 & -2 & 12 & 160 \\ 159 & 10\end{array}$

$-10-212$ 46 52 43

$\begin{array}{lll}-9 & -2 & 12 \\ -8 & -2 & 12 \\ - & 59 & 22\end{array}$

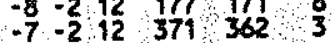

$.1 \%$ का

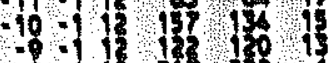

-111200

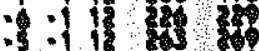

- -1 11212

$-1130$

010 )

- 11 II 105

112 ?

-15 0121416

-16 6 of

-18 8130151

- 01110110

- 12 kद

18121615

30126\% 2

- 12 123 128 10

- 17100

- 112168 160

$-1126574$

o $12 \quad 57 \quad 51$

\begin{tabular}{ccccccc}
6 & 12 & 04 & 86 & 18 \\
\hline & 12 & 56 & 50 & 55
\end{tabular}

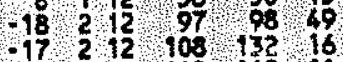

-16 2 12 146 148 11

-14212 225 227

$-13,212,161,183$ ? 12

$\begin{array}{llllll}-11 & 2 & 12 & 112 & 103 & 11\end{array}$

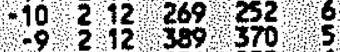

48212 254 259 . 6

$-6 \quad 212 \quad 377353$

$-5 \quad 212 \quad 293270$

$\begin{array}{llllll}-4 & 2 & 12 & 62 & 53 & 18\end{array}$

-2 2 12 149 $154 \quad 7$
- 1120376 h $\quad$ lopo 10\% 100

is 12 20

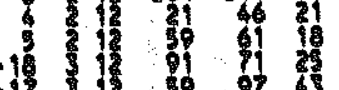

7 . If 19 is 30 or

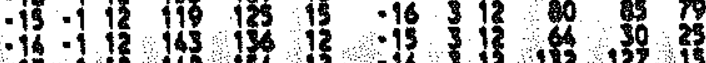

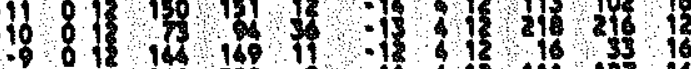

$-8122011212$

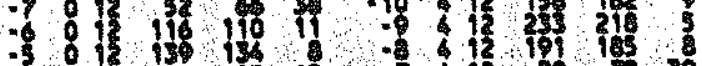

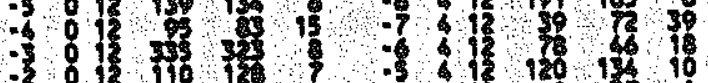

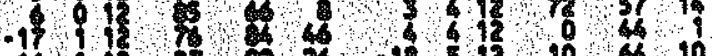

\begin{tabular}{llll}
3 & 12 & 102 & 190 \\
4 & 274 & 266 & 5 \\
\hline
\end{tabular}
561892

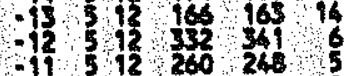

- 212515

112323

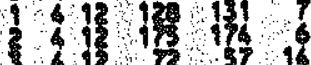

$185,10 \% 30$

-16 512 123 11511

-11512 260 155

-91210015
$-7512 \quad 166135$

$-7512209$

$\begin{array}{lll}-6 & 12 & 138 \\ -5 & 512 & 14\end{array}$

- $512 \quad 2018210$

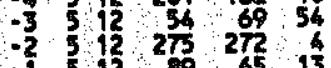

-1 $512 \quad 89 \quad 65 \quad 13$

$151266 \quad 59$ 18

2 512,76 . 1315

$176 \quad 612,163,161$ \%?

$-95612,77$ 87 24

-13612 314 305 ,

$-12612,43,6943$

-10.612 . 64 , 74,63

8612182189 ?

$-7612 \quad 223$ 220 13

-5 $612 \quad 59$ क 32

$\begin{array}{llllll}-4 & 6 & 12 & 242 & 234 & 7 \\ -3 & 6 & 12 & 40 & 73 & 39\end{array}$

$\begin{array}{lllll}-2 & 6 & 12 & 158 & 154\end{array}$

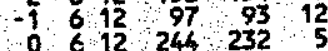

$-18612,145,129,12$ ho $110 \% 0$ 10Fe $10 \mathrm{~s}$ $-171 \%$ का -15 7130 \% -12 7213 14 -11 $712 \quad 76 \%$ - 717716016 6712141132 .77197018 .71 153 13 .7160 - 071532 1871 of 3739

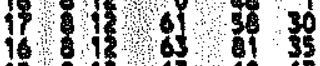
$-15816360$ $13012 \quad 301 \quad 300$ 11 12 al 90 री - 13100168 iा - 912030 - 3151621328 - 11243 of 69 - का 151 - 137 $176120 \%$ 15 1202 20 10 -13.13 20700 $11 \% 1215715512$ $10,13{ }^{6}, 16023$

-8 9110010 -7.9 102 10 17

- 912 क 69

$-912123 \quad 123 \quad 12$ -2912120 126 12 $-18101230$ $-171012,6963$ $-161012,263$ 255 6 -1610 13142 10 1216 - 11 10 24326210 $.91012 \quad 54 \quad 865$ $\begin{array}{lllll}-8 & 10 & 12 & 19 & 7 \\ -7 & & \end{array}$ $-71012 \times 61 t^{5} 28$ $\begin{array}{llll}-610 & 12 & 0 & 13\end{array}$ 41012 . $47,47,46$ $21012,109,114,92$ $-171112, \quad 71,10170$

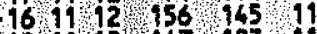

$-1511271471235$ $-131112 \quad 133$. $125 \quad 12$ $-121112 \quad 293 \quad 297537$ $-101112,144 \quad 13517$ -9 $11112,227,232,6$ - 71112161 164 ? $\begin{array}{llllll}-5 & 11 & 12 & 71 & 78 & 48 \\ -4 & 1 & 12 & 65 & 72 & 20\end{array}$ -411120023 व $\begin{array}{llllll}-17 & 22 & 2 & 68 & 68 & 42 \\ -16 & 12 & 12 & 52 & 38 & 52\end{array}$ 


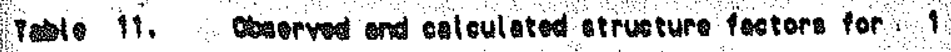

Paed 28

क 110901096105

ข1 $1211 \%$

t)

40 12 190

T. If is is

- 15 110 ing

1513

I. 1019

II 11 ig

$10,12 \% 67$

को

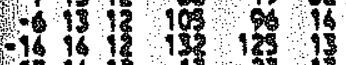

13 is 13 11 12 , 150 10 40.6135 का $1.913 \% 35$

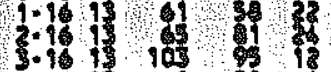

-6.15 I 00

+on 00150

-1.15 is

1.15 19 15

3.13

-15 3575

-1 13

- -15 I I 19

- -14 I 110 119 11

-1.13 o

1-1 15 o 15

3.1 I 110

5-1 1 100 क 10

-7.141 \% 35

- 6.13 I $110 \%$ \% 16

- 4 - 13181

$-3.1313150$

$\begin{array}{lll}-1.13 & 140 \quad 136 \quad 6\end{array}$

$\begin{array}{lll}0.13 & 20 & 15 \\ 1.95 & 519\end{array}$

2. $1312117 \quad 8$

6.1313162 134 13

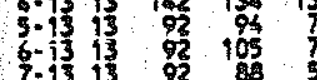

$\begin{array}{rlll}-8-12 & 13 & 3 & 5\end{array}$

-7.1213 136 136

$\begin{array}{lllll}-6-12 & 15 & 125 & 136 & 17 \\ -5-12 & 93 & 89 & 77\end{array}$

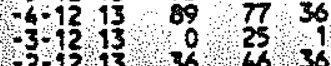

$-2,12,13,36,46,36$

$01121313114,101.8$

2. 3213 13 201 200 87

$\begin{array}{llll}4-12 & 13\end{array}$

$6.9213 \quad 56 \quad 59$ ?

$-91113,00036$

-7.193410 ? 10

\begin{tabular}{lllll}
$-5-11$ & 13 & 31 & 36 & 30 \\
\hline & 86 & 97 & 15
\end{tabular}

$-5.17 \frac{13}{6}, 56,97,15$

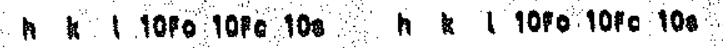

- I.1 1192

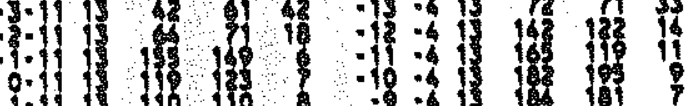

2.11 1196

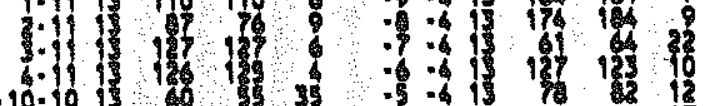

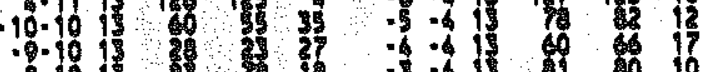

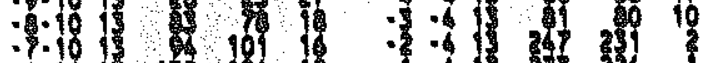

- 10 19 101

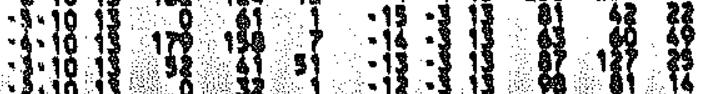

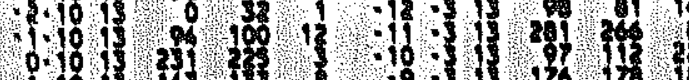

1.

$19.10 \% 207$

10.91506

- -91169 1 3

$.7 \% 1320320716$

-6. 615162137 .

$-6139010416$

- 61 1 1916

o 12135

.12 .1365

-10.1 83

- -13100

$-7-111315011$

- -131917

- -8130156

- - - 13 16/13 6

- -1320135

1 - 16121

$\begin{array}{ll}-13 & -7 \\ -11 & -7\end{array} 140$

$-90-7156510$

- $-7.13,169,16011$

$\begin{array}{lll}-6 & -713 \\ -3 & -7 & 3\end{array}$ 20 35

$-6-71311813610$

$-2-713105116$

$-1-713$ 212 210 2

$-16.63565$

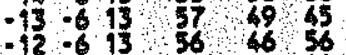

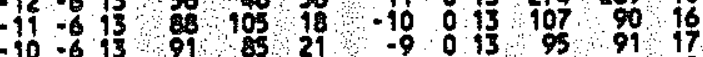

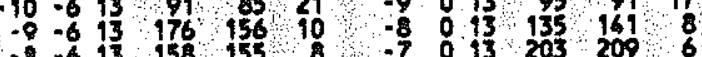

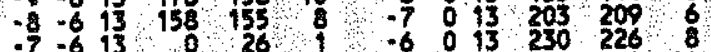

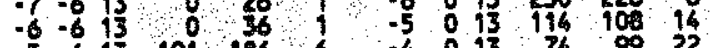

$\begin{array}{lllllllllllll}-5 & -6 & 5 & 191 & 186 & 6 & -4 & 0 & 13 & 74 & 99 & 22 \\ -4 & -6 & 3 & 84 & 71 & 13 & -3 & 0 & 13 & 106 & 124 & 90\end{array}$

$\begin{array}{llllll}-4 & -6 & 13 & 84 & 71 & 13 \\ -3 & -6 & 13 & 246 & 255 & 3\end{array}$

- 2 -6 is $156 \quad 165$

$-10613,219,204,6$

$13513166,174,13$

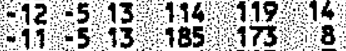

-10.5 $13,205,213,7$

$8.513,98,9214$

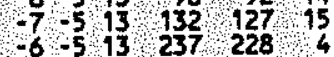

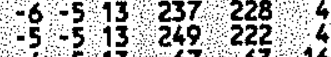

$171613,213,210,15$

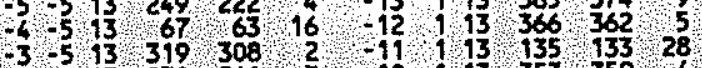

$\begin{array}{cccccc}-3 & 5 & 5 & 13 & 319 & 308 \\ 2 & -5 & 3 & 230 & 244\end{array}$

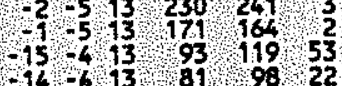

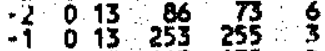

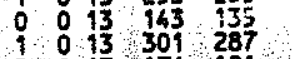

$\begin{array}{llllll}2 & 0 & 13 & 176 & 181 \\ 3 & 0 & 13 & 228 & 220\end{array}$

3 013 13 $102,115,6$

$\begin{array}{lllllll}5 & 0 & 13 & 27 & 36 & 26 \\ 17 & 13 & 16 & 23 & 15\end{array}$

$\begin{array}{llllll}-15 & 15 & 13 & 66 & 65 & 30 \\ -14 & 13 & 234 & 238 & 6\end{array}$

ค 1 10po loge 100

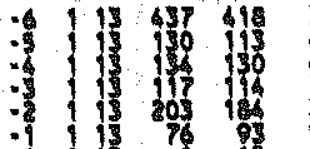

111975 17

6 of

-16 145 190 11

-15 15 15 19

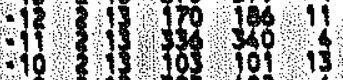

.013303402

- 2 1 1 10 10 11

- 51310 14 is

- 21316028

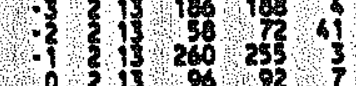

31317102

31509611

$-1131{ }^{13} 160$

-15 151100

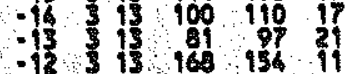

$-12313150350$

- 13215250

$-7313113013$

- 31305

- 313 20 sos

-113 1 52152

1313019727

2. 31516112,7

$\begin{array}{lll}-18 & 13 & 60\end{array}$

$.16 \% 1376931$

$\begin{array}{llllll}-15 & 13 & 116 & 104 & 14 \\ -16 & 13 & 91 & 54\end{array}$

- 13 \& 1316,2267

-11 413 28 283

-9 413133130

$\begin{array}{llll}-8 & 13 & 15 & 46\end{array}$

$\begin{array}{llllll}-7 & 13 & 183 & 185 & 7 \\ -6 & 13 & 295 & 292 & 3\end{array}$

-5 13 150 $131 \quad 13$

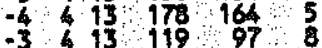

$\begin{array}{llllll}-2 & 4 & 13 & 236 & 235 & 4 \\ -1 & 4 & 122 & 107 & 26\end{array}$

$\begin{array}{ccccc}-1 & 45 & 122 & 107 & 26 \\ 0 & 13 & 262 & 269\end{array}$

9413 o 1619

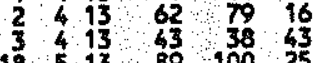

$\begin{array}{rrrr}-18 & 5 & 13 & 89 \\ -17 & 5 & 13 & 134\end{array}$

-16. 513210 197?

$-15513,15,121,14$

- $135^{13} \quad 242 \quad 242$. 12

$\begin{array}{ccccc}12 & 5 & 13 & 151 \\ -11 & 5 & 13 & 164 & 72\end{array}$

$-10 \quad 513 \quad 235214$. 7

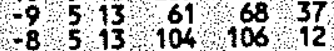

$-7 \% 13$ 92 72.13

$\begin{array}{llllll}-110 & 13 & 1353 & 359 & 28\end{array}$

$\begin{array}{lllll}-6 & 5 & 13 & 148 & 148 \\ -5 & 5 & 26 & 58 & 26\end{array}$

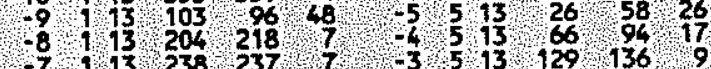

h 1 lofo lope los

- 13 स 130 12

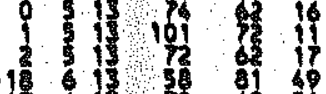

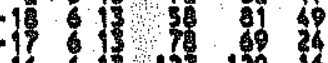

- 15 1 13? 120 is

-14.107 107

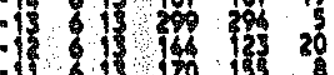

-1061216 225

- $81527 \%$ 21 7

.611353513

$-3613515021$

-2613 2002115

1613 o क क 12

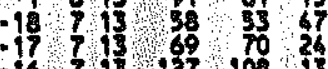

.167127 108 13

-167) 151 i37?

1571527275

$.1071,132,130$

- 71 la 1013

$-6752362$

-513230 256

-713 - 790 \% 3

$-27116317$

$.071 \% 503$

-1781300 क्ष

- 151391 क

-13 a 17161

$-118132925$

- 81315016019

$-8 \quad 8132662695$

6831651648

$\begin{array}{llll}-5 & 8 & 37 \\ -6 & 8 & 87 & 32\end{array}$

$\begin{array}{lllll}-3 & 813 & 186 & 168\end{array}$

- 813128128 ?

-18
-17

-16913185 188 11

- $15 \% 15 \quad 66$ 20 25

$-13913 \quad 82 \quad 6021$

- $911 \quad 9 \quad 25725615$

$10 \quad 93158 \quad 156 \quad 10$

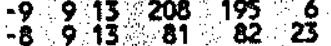

$-79131551616$

$\begin{array}{llll}-6 & 9 & 13 & 0 \\ -5 & 9 & 148 & 14\end{array}$

$\begin{array}{llll}-4 & 9 & 13 & 242 \\ -5 & 230 & 9\end{array}$

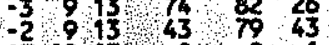

$\begin{array}{rlll}17 & 1013 \\ -16 & 0 & 0\end{array}$

$151013,66,79,25$

$\begin{array}{rllll}-13 & 1013 & 179 & 156 & 10 \\ 56 & 80 & 56\end{array}$

$-1210 \mathrm{13} 384,383,20$

1110132942835

$-91013 \quad 296728$

$810,13,92,88,16$

61013191202 12

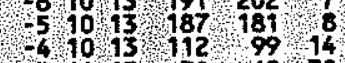

$\begin{array}{llllll}4 & 10 & 13 & 112 & 99 & 14 \\ 3 & 10 & 30 & 69 & 30\end{array}$ 


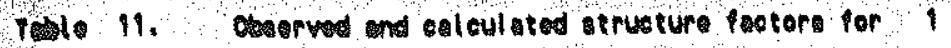

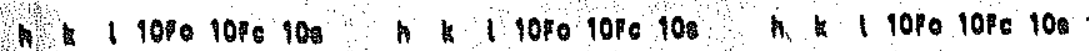

01711 is 1919

+1)

1) 1130 ?

101113 is is

$+\% 1113019$

$\rightarrow 19$ i

-1911 0

-151 1 5 क

$1311 \% 10 \%$

91612003

- 121101031

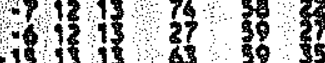

-14 15 13 135 19517

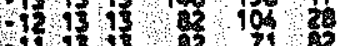

- 9131 , 123,31

$0.1516,554$

0.151313

8.15 is is it 3

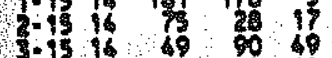

- 15 is 100119

$\begin{array}{ccc}-3.14 & 0 & 10 \\ -4.96 & 70 & 10\end{array}$

-3.14 16 क 1219

$+1416 \quad 0 \quad 376$

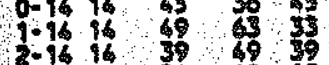

3-16 96496

-14 10309

-14 16 ? 137 ?

- -1414 19 113

$+1510010$

II is 14015 ?

1130 का

\&-13 19 190

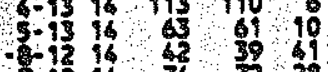

- $6-12$ 14 $64 \quad 72 \quad 28$

$-5-1216 \quad 0$ 156

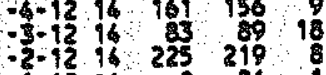

$-1.1214 \quad 0 \quad 26,15$

1-12 if 103108 .

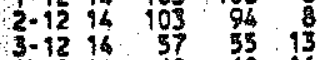

$61216 \quad 60 \quad 0016$

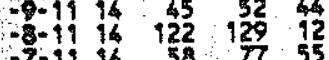

$61114 \quad 93 \quad 10343$

$-5-1116$ 16 177205

$-3.1149132,116$ 10

(2) 1.14 116,121 .

$0.1116,170 \quad 167$.

21) 16112 101 20

30 1101459 58

9.10:147 851

8.10 14 54 त59 54

$\begin{array}{rlll}-6-1014 & 190 & 83 & 11\end{array}$

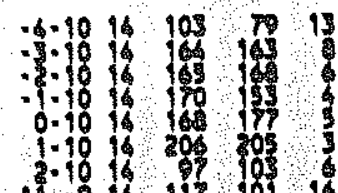

2.10 16 of 1170

: 10 औरूद

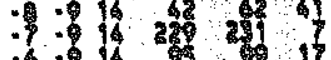

- 101

- - 14 12 109

- -91473725

9.9.6315 310

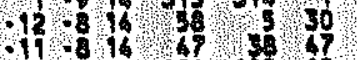

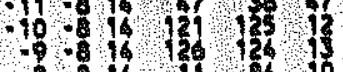

- 810 I1 110

- 6 . 15 क 15

. 8116402203

-3.814 15 127 10

161446,15

$-13-716$ 16 1093

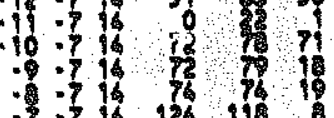

$-51610710 \% 1$ o 10 is of

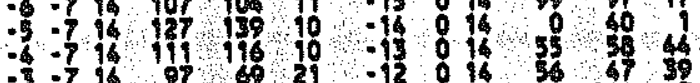

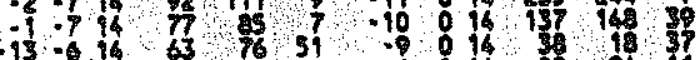

$-12.610010515$

$-11.6110109$

$-914101010$

.014101019

61200192

$-5=6149129$

- -61 का दो

- -1.16113193

- 16.51414514

- 10.516103 १ 97

-9 $-516 \quad 0 \quad 13$ का

-7.5 9 \% 13

-6.5 $14 \quad 267 \quad 26016$

- .5 1616016013

$\begin{array}{rrr}-3.5 & 16 \quad 173 & 196\end{array}$

$-1.514 \quad 264256 \quad 57$

$-15.414 \quad 58 \quad 7157$

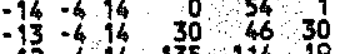

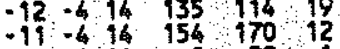

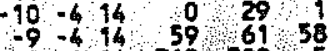

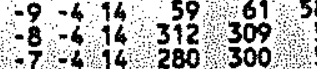

\begin{tabular}{llll}
6 & 414 & 280 & 300 \\
\hline
\end{tabular}

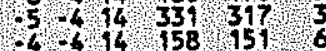

$3.619,134,1456$

$\begin{array}{rrrrrr}2 & 614 & 81 & 85 & 6 \\ -15 & -3 & 62 & 81 & 61\end{array}$

$-14314 \quad 196 \% 196,8$

$\begin{array}{rrrrr}13 & -3 & 14 & 142 & 162\end{array} 111$

41314 79 6197,78

$\begin{array}{lllll}10 & 3 & 14 & 82 \\ -9 & -3 & 14 & 281 & 70\end{array}, 15$

- $14 \quad 202 \quad 21522$

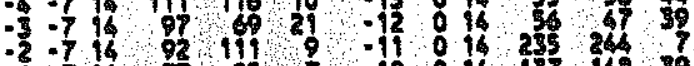

- 2 - 510103130,16

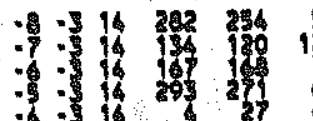

. : 16 14 14

浩 19090

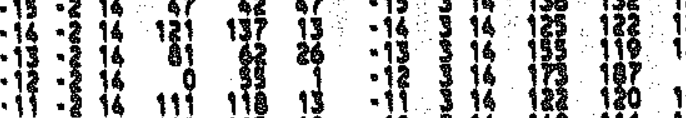

.

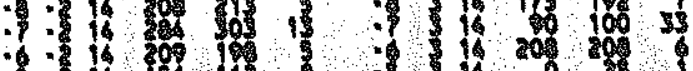

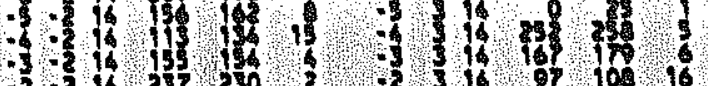

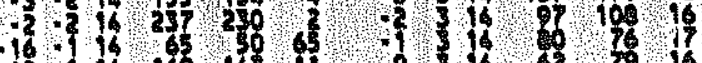

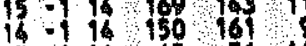

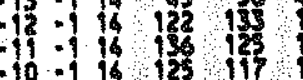

-16. 160 का 2

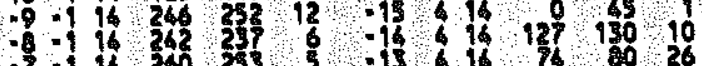

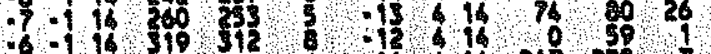

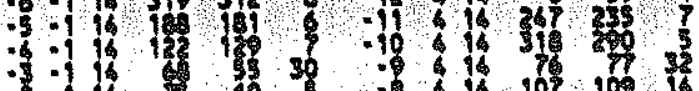

.

-7 is 1109

- 11 i1s 100

- $\left.3161^{6}\right)^{21}$

$-1142602710$

.016210216

- 60140700

. 0 1 1210

- 0161616

0016169

$-170^{14}{ }^{7}$ का

- 16 1 $128 \% 5$

- 15 If 111116

- 131216 las 16322

.11 14 165 176

$\rightarrow \quad 1 \quad 222 \quad 22$

$\begin{array}{llll}6 & 16 & 156 \\ 333 & 344\end{array}$

$\begin{array}{lll}-4 & 14 & 250 \\ -3 & 149\end{array}$

$\begin{array}{lllllll}-2 & 14 & 14 & 68 & 59 & 15 \\ -1 & 14 & 25 & 38 & 25\end{array}$

011507015

$\begin{array}{lllll}1 & 14 & 84 & 78 & 7 \\ 3 & 14 & 280 & 290\end{array}$

$3114114 \quad 112 \quad 5 \quad 0616 \quad 65$ 54

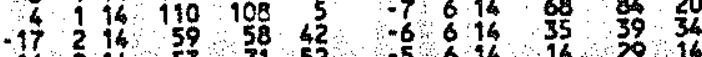

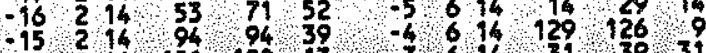

$-16214121,100,13$

$42,14325,334,5$

$2.616132,136,3$

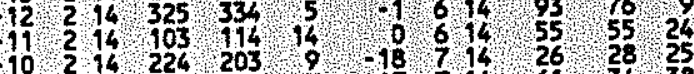

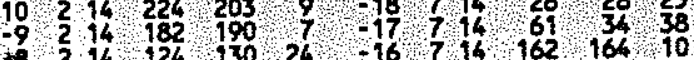

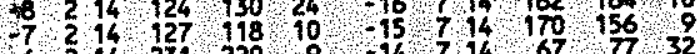

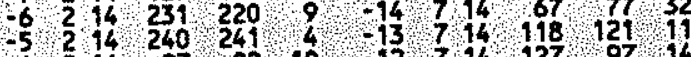

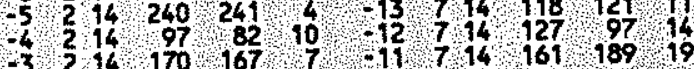

A l loFo 10F 10

$-1071400602$

- 71911610 is

- 7 16 101 \% 18

- 711171011

- 71656

-17 $16 \quad 0013$

- 15 15 145 का

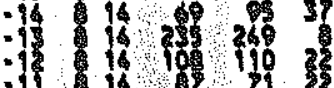

$.1116 \%$

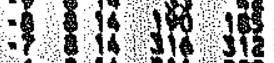

.61634129

- 614 10 120

$.17 \%$ is 32 s 52

.16 .9645 औ 53

14216123 14 13

1201620

- $10.140 \pi$ 107.

- 1635

- 1463

. 14 \%

- 316445

- 16101110120

- 15 10 113 is 1

- 1516 150 56

- 11101416919

-910 0 so

$-11016120109$

- 10 15 13

.5 $101 \%$ का

-111 190

$1311960 \%$

- 11 1 121 116 17

.119097420

$-7111640046$

\begin{tabular}{lllll}
-6 & 1 & 16 & 0 & 35 \\
\hline 5 & 2 & 16 & 23 & 65
\end{tabular}

14 1216 \$1 102 :3

- 1214 क 15 ।

$-11121415014611$

$-91216 \quad \frac{15}{0} 1 \frac{22}{25}$

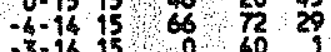

$-2-14 \quad 15112 \quad 110$

$-1+1415,130 \quad 120$

1.145112093

$3-1415 \quad 04 \frac{3}{5} 11$

$\begin{array}{llll}-6-13 & 15 & 7 & 36 \\ -5-13 & 6 & 6\end{array}$

$\begin{array}{r}5.13 \\ -13\end{array}, 612,73,61$

-313 15 168 159. 8

$\begin{array}{rlll}-2-13 & 15 & 121 & 117 \\ 132 & 121\end{array}$

$01 \frac{13}{15}, 0,17 \%$

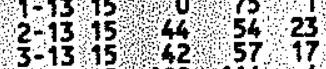

$4-1315,109,114,4$

$-7-1215.86,5822$ 


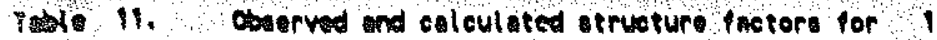

Peg 24

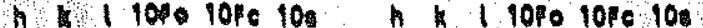

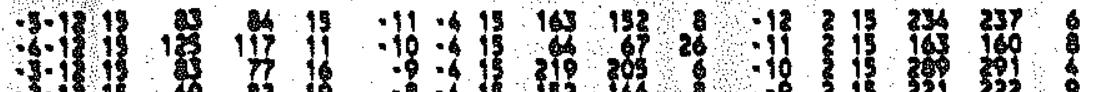

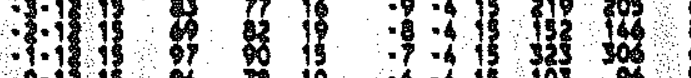

9.13 35

a.1 1610

- -1111010

$-5=11113$ is 105

- $=15$ of

-1.1) 1616

0.11515160

3.11130 . 17

81015 \% 12

- .1015 in 174

- -1955

- 2.101521923

0.1915 का 120

$.10 \% 45979$

8.91 103

- -9 213210

- -915 17 ln $^{3}$

6.9132 ls 6

$+813160157$

1 159

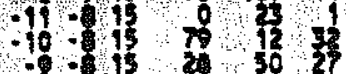

- $16152^{13}{ }^{130}$

- -6j 140

- -115130

- -11 का

o-6 का 19

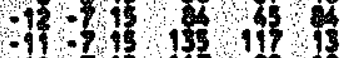

$-10-715$ 109 971

-715
-715 10718

- 715212

$-5-7151900$

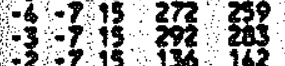

$-13-615072$

$+13-615$ 10 $171 \quad 16$

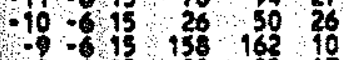

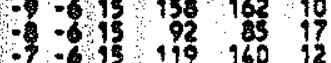

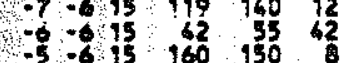

$\begin{array}{llll}-5-6 & 160 & 150 \\ -6 & -6 & 205 & 253\end{array}$

-3-615 259 253

$-16-5151119419$

$3-515$
$-12-515$

61105j 5549611621

$.6515,126,125$ 12

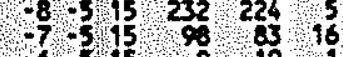

-6.515 1601479

-5.515 160 147 219

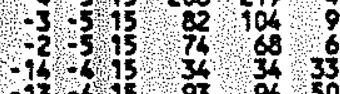

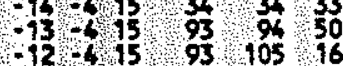

- 61 los 10

- 5131119

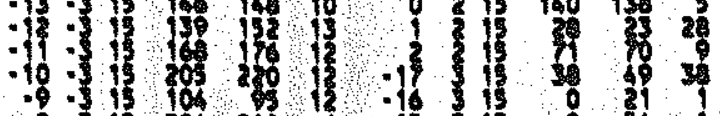

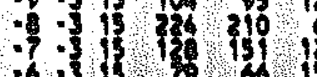

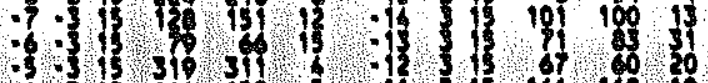

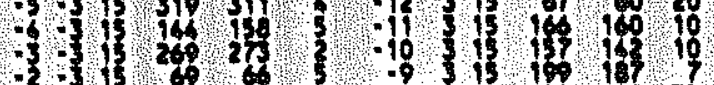

$-3,251063$

-16.2 1500 in 18

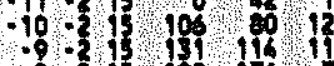

.6 .31510976

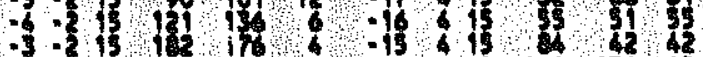

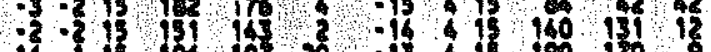

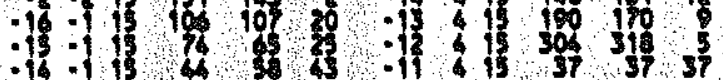

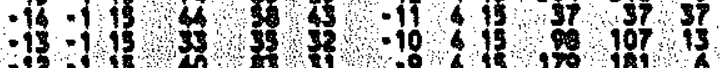

$.12-11320 \% 13$

$-10.115360$

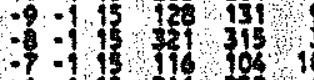

6.11360

$-11 \%$

1.9173073

$.16 \% 141319$

-16 013 का 13

.120151315

.1001364

- 015253

-7 \% 1316011

- 0 का $281260 \% 13$

$-601512419$

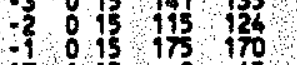

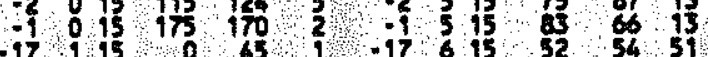

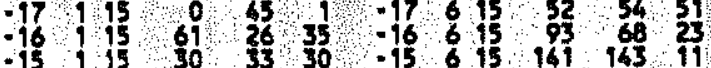

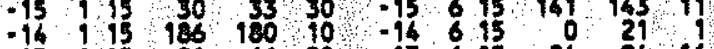

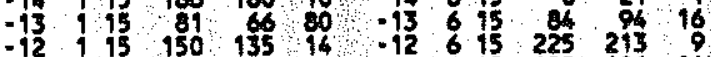

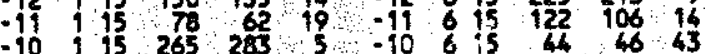

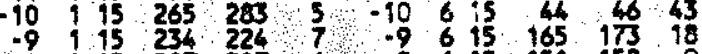

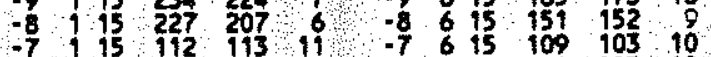

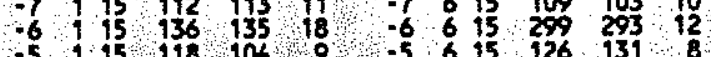

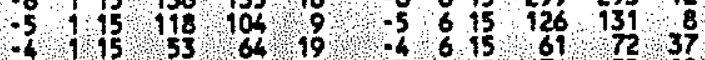

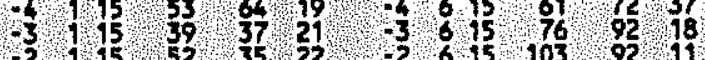

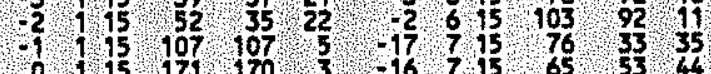

\begin{tabular}{lllllllllll}
0 & 15 & 171 & 170 & 3 & -16 & 7 & 15 & 65 & 53 & 44 \\
\hline & 27 & 43 & 27 & -15 & 7 & 153 & 129 & 18
\end{tabular}

$\begin{array}{cllllllllll}2 & 17 & 15 & 48 & 37 & 9 & 16 & 7 & 15 & 253 & 252\end{array}$

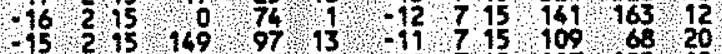

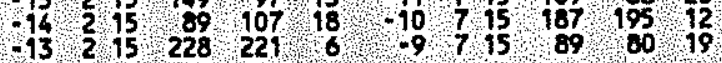

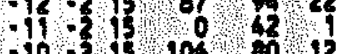

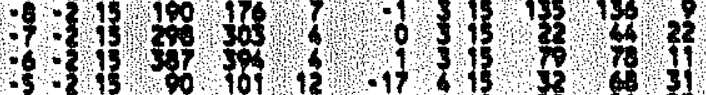

a 1 10po 10Ro 100

-7 713 319 302

- 715 \% 750

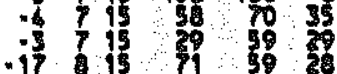

$.17 \% 161019$

11 15106140 is

-13 is 1122

-10 is 1940

- 15 is 131

- $6150 \%$

$.4 \% 1503$

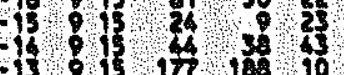

.2 .1510218710

-11915 \% 10019

-9
-89

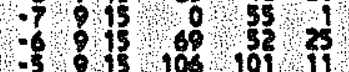

-161015 108 101 \% 11

-16105 का 1473

-12 15 I 126

-11 10 क 207 os 10

- 1012 I0 1

- 101517610

$-14151191$

$-11136203$

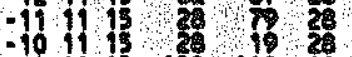

$-11131901911$

- -1316 113 $10 \%$

-1.1316123 , 137

1.16465027

${ }_{6}^{2-12} 16 \% 196$

-5. 1216 2 $25 \%$

$-4.1216 \quad 111 \quad 9716$

$\begin{array}{llll}-3-12 & 16 & 7 & 7\end{array}$

- -1216126 121 9

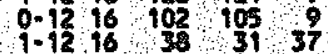

- $2-1216,17,36 \quad 17$

$.7-11$ 16 810819

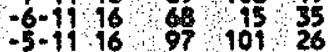

$-6-119695 \%$

$-2.1196136126$

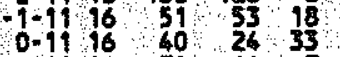

11167 o 7

$-8.1016 \quad 65$ os 21

$=7-1016 \quad 967723$

$\begin{array}{llll}-3-10 & 16 & 76 & 73 \\ -5-10 & 76 & 71 & 53\end{array}$

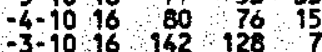

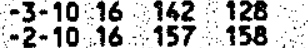

$-1-10616126125$

0.1016113109

9016 . 0 . 65 \%

79,16 , 62 24 62

$-5-916,58 \quad 8126$

$4-916,77$ 88 14

$-3916 \quad 175,173,5$

$\begin{array}{lllll}-2 & -9 & 16 & 65 & 70 \\ -1 & 9 & 16 & 156 & 159\end{array}$ h l lopo $10 \%$ o 100

0.9

$=110$ : $16 \quad 18$ \% 40 .8 .816 of 103 -7.816 ग 10 - -16172158 - - 16 167210

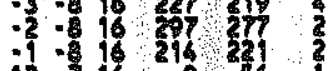

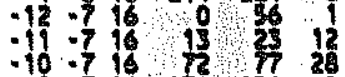
$-10.718 \quad 72,77$ - 716 10 110 .7 .716 las 178 $-5-716126131$ -3.716 18 1976 -13.616 o 55 $-11.616135 \%$ \% 12 $-10.616 \quad 21 \times 19720$ - -6161790219

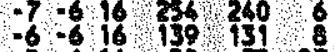
$.5-616$, 56 , 12,27

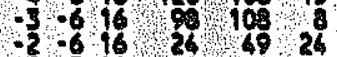
$-15.516 \% 0 \% 18$ - 11.516 का 16405 -9.6 II of is $-7.51616163$ $-6.5162121955$ $-3.516 \quad 55 \quad 67 \quad 55$ - 3161051056 $-2-316 \quad 219,229,37$

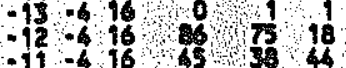
$-10-416$ 215 222 ? $-9-616135125,16$ -7.416227226 s $-6.6162051209,8$ $\begin{array}{lll}-5 & -4 & 16 \\ -563 & 369\end{array}$ $-3=16 \quad 17616810$ $\begin{array}{rrrl}-15 & -36 & 16 & 0 \\ -13 & -3 & 16 & 0\end{array}$ $\begin{array}{rrrr}-13 & -3 & 16 & 0 \\ -12 & -3 & 56 & 36\end{array}$ $\begin{array}{rrrr}-11.316 & 157 & 160 & 25 \\ -10.3 & 16 & 211 & 202\end{array}$ $-9-316 \quad 112$ 125 10 $\begin{array}{lll}-8 & -3 & 16 \\ -7 & -3 & 164\end{array}$ $\begin{array}{lllll}-6 & -3 & 16 & 105 & 101\end{array}$ $-4-316$. 169 \& 139

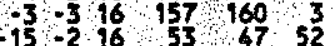
$-16-216,0$. 19

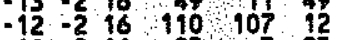

$\begin{array}{lllll}-11 & -2 & 16 & 25 \\ -10 & -2 & 16 & 162 & 3\end{array}$ $-9-216 \quad 173 \div 174 \quad 7$

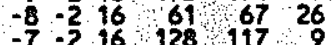
$-6=-216 \quad 236,233,4$ $\begin{array}{llll}-5 & -2 & 16 & 43 \\ -6 & -2 & 43\end{array}$ $\begin{array}{llllll}-6 & -2 & 16 & 171 & 166 & 4 \\ -3 & 2 & 16 & 28 & 36 & 28\end{array}$ -15416 78 39,18 $-14,16$, 50,4446 $-11-116,218,212$. 8

$-10-116 \quad 104,9220$ $-8-116 \quad 131$ 125 11 7416128,113 ? 
Pene 85

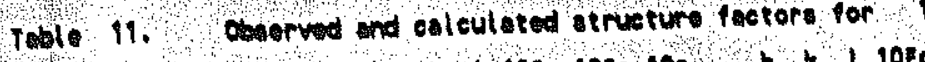

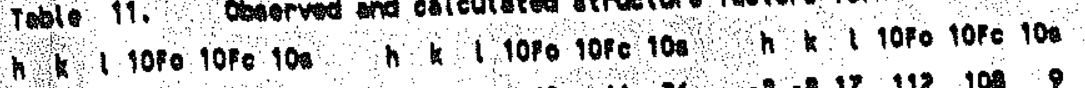

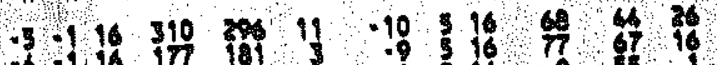
-31 los 150 - -7 .16816 o 16017

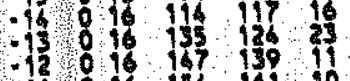
011016 is 15 .98162210 $.68 \%$ b3 31 601625 23 25 -3. 016162123 $-161516 \quad 67,32$

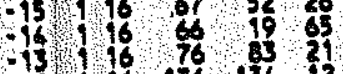
$+12196136 \quad 134$ 12

-10.16 112 114 $-711637339$

$-616167 \%$

-3) 16 का 120

$-21,16$ 162 150

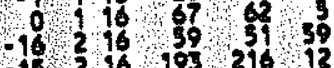

-1526 193 11612

$\begin{array}{rlll}13 & 26 & 76 & 45 \\ 2 & 26 & 26 \\ 2 & 16 & 13\end{array}$

$-10216 \quad 1531915$

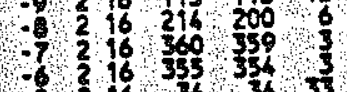

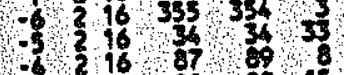

-62 216 80 92 का5

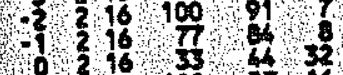
$416316,90057,16$ * 19316 as 1392

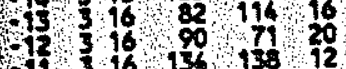

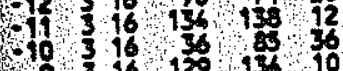
- 10,16 170 1310

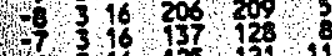
6316,12512111 $4616, \quad 57 \quad 62,33$ T2 316 18 116,7 $\begin{array}{lllll}0 & 3 & 16 & 61 & 63\end{array}$ $96 \quad 16 \quad 162 \quad 130 \quad 13$

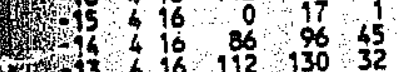

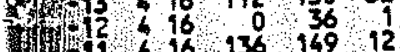
$40416 \quad 126,198 \quad 17$

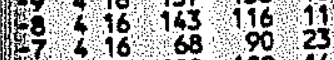
$4.796180,189,14$ (196) 46,827290 346,225230 ?

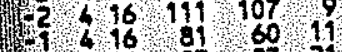
$47 \% 16,83,8321$ 17 5 16 15 45,14

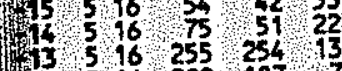

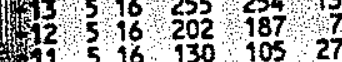

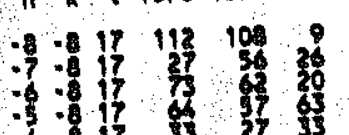

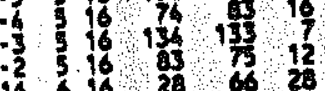
$1616 \%$ का - 16160136123

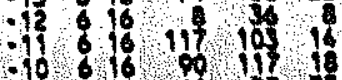
$10.16,961831$

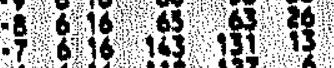
.6.616 $9137 \%$ $-56161904016$

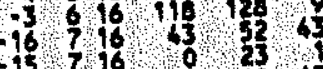
$-15776 \% 45 \% 9$ -13.716 710016318

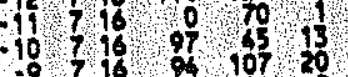
-916
-7716130 $-6716,14,45$

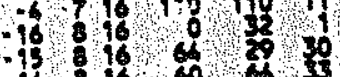

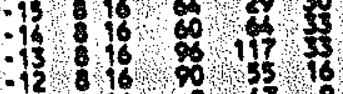
$-11816109469$ $-881612613210$ $-7816,58+1818$ -6816 \% 137311 -1496 \% 90425

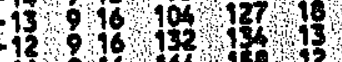

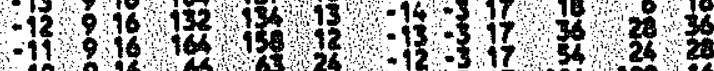

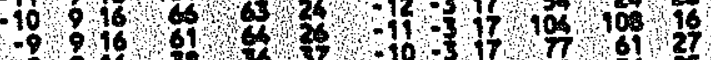
-896
-7.96 $-131016$

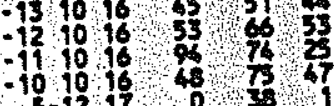
- -127711811110 $-3-1217,115,126,105$ $\begin{array}{ll}-2.12 \\ -1-12 & 17 \\ 0 & 65 \\ 6\end{array}$ $0.1217,64,6311$ $-6.1197226223 \% 6$ - -1117777739 -2.1117 120 12327 $0-111789896$

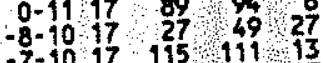
$\begin{array}{lll}-7-10 & 17 & 115 \\ -6-10 & 17 & 89\end{array}$ $\begin{array}{llll}5-10 & 17 & 63 & 34\end{array}$

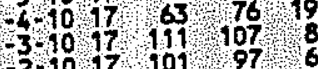
$\begin{array}{lllll}2 & 10 & 17 & 901 & 97 \\ -1 & 10 & 62 & 61\end{array}$ 0.1017 22 5521 $\begin{array}{llllll}9.9 & 17 & 70 & 47 & 27\end{array}$

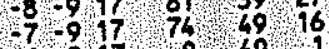
$\begin{array}{llllll}6 & 9 & 17 & 0 & 40 & 1\end{array}$ $4.9,77126.038$. $\begin{array}{lllllll}4 & 4 & 9 & 17 & 0 & 0 & 38 \\ -3 & -9 & 69 & 13\end{array}$

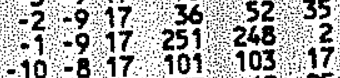
$\begin{array}{llllll}-4 & -2 & 17 & 138 & 124\end{array}$

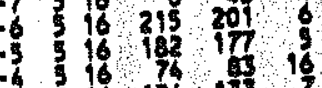

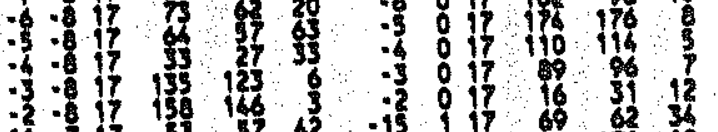

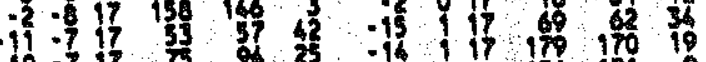
$=-71792$ os

$-7.717 \quad 216$ 20 11 $-6=777 \%$ $-71716106-7) 7$ b3

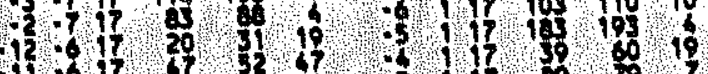

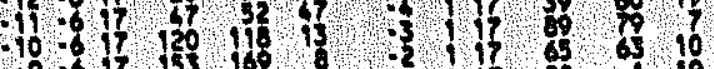

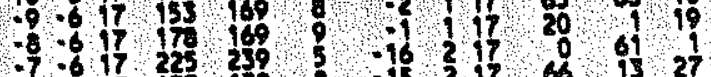

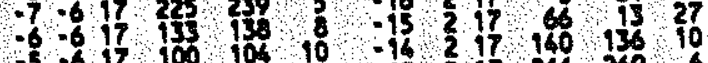

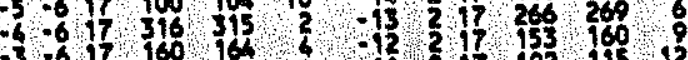

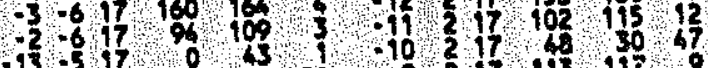

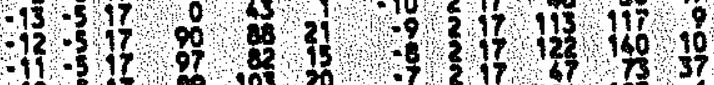

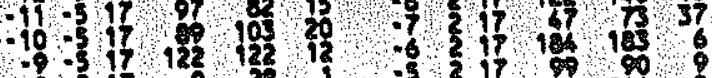
$\begin{array}{lll}-6 & -5\end{array}$

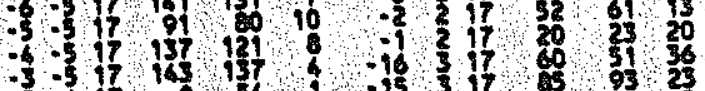

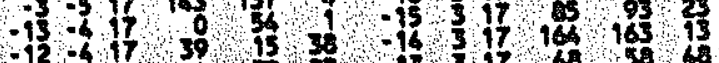

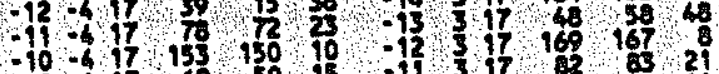

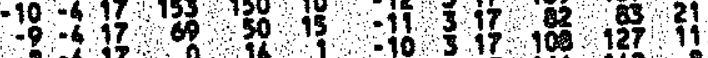

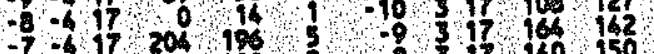
$-6171651095 \% 317,1601507$ -5.617 of $72,0-63171271319$

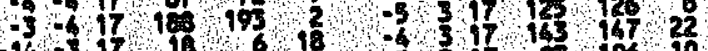
- 317 का 10610 -16417 क 6830 -15417 1301 122 28 $-1361716613512$ -11 $17,0 \quad 12710$ - 4710033 -847110 16 31 $-7.17,1237515$

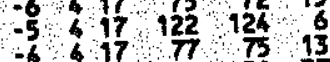
- $\quad 47$ \% $37 \quad 32 \quad 37$ $\begin{array}{rrrr}-16 & 5 & 7 & 133 \\ -1 & 5 & 106 & 12\end{array}$ $\begin{array}{llll}-15 & 517 & 78 & 64\end{array}$ $\begin{array}{lllll}-13 & 5 & 17 & 86 & 86 \\ -12 & 17 & 118 & 109\end{array}$ $\begin{array}{llll}-11 & 5 & 17 \quad 87 \quad 94 & 30\end{array}$

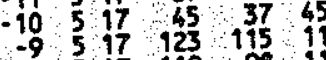

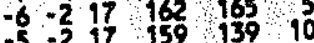
$\begin{array}{llll}-3 & -2 & 17 & 171 \\ 0 & 197 & 24\end{array}$ $-14-17 \quad 93$ \% 21

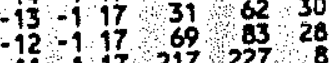
$-11,17217$ 227 32 $09,17,64,94,24$ $4-117165163$. 6 $0 .-117963,160,11$ $\begin{array}{ccc}-5 & 17 \\ -4 & 17 & 92 \\ 86\end{array}$ 31172382302 $-15,0,17,47,49,46$ $-13 \% 1781,87,24$

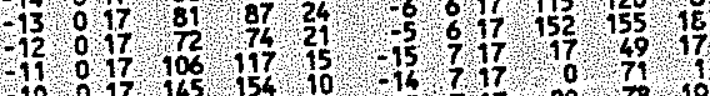

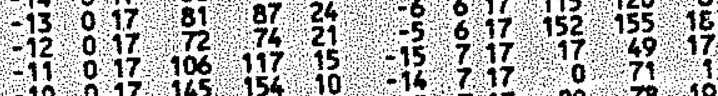
1571775744917 h. $118 \% 010 \% \mathrm{e} 100$ 12717 is 10016 .9717 10 11 .7717 of 35 .797969 -15 17 \% 648 -11 $817,00 \quad 100$ \% 17 123 12 - -11 125116 $.610160 \%$ .510 40 ? 40 30110110 -1n10 10 69 55 10 $-6.918776428$ -6.91837 49 $-5.91640432$ 3.91669714 -2.918 50 36 26 6.866969 $-6.81823 \quad 46$ -5 - 16 of -2.618 \& 3720 $-9-7836$ \% $.7-71815$ । 150 $-6-71813019716$ $-577130126$ $-3-78$ \% 1 $-11-618001210$ 9.6 106 7 $-8.61810130$ -6.618 \$0 6140 उ० 100116 $-3619768$ $-11 \% 1866$ \% 45 $-9-518104102,20$ $-8-518117$ । 130

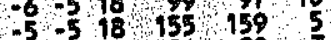

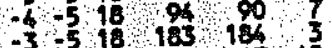
$\begin{array}{lllll}-3 & -5 & 18 & 183 & 18 \\ -12 & -4 & 46 & 45\end{array}$ $-114581905658$ $-9-41800 \quad 9512$ $-8-41840$ 35 39 $-7-618$ 9 79 is $-6 x^{-6} 88122126 \quad 5$ $-4-6181161215$ $-13-31854545$

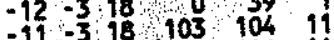
$-10-318111$ 101 14 $-8-318 \% 0 \quad 29$ $\begin{array}{lllll}-7 & 3 & 18 & 64 & 52\end{array}$ 5 - - 18 196 181

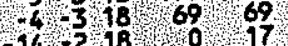
$43-218,0$ \%

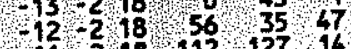
$\begin{array}{ccc}-11 & -2 & 18 \\ -10 & 112 & 127\end{array}$ $\begin{array}{rrrrr}-10-2 & -28 & 99 & 72 & 17 \\ -8 & -2 & 18 & 134 & 138\end{array}$ $\begin{array}{rlll}-8.218 & 134 & 138 & 8 \\ -75 & 18\end{array}$ -6.2 165174 \% \begin{tabular}{llllll}
\hline 5 & 2 & 18 & 128 & 132 & 6 \\
-4 & -2 & 18 & 96 & 90
\end{tabular}

$-9-817-73$ क 625 


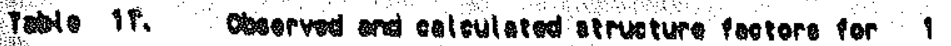

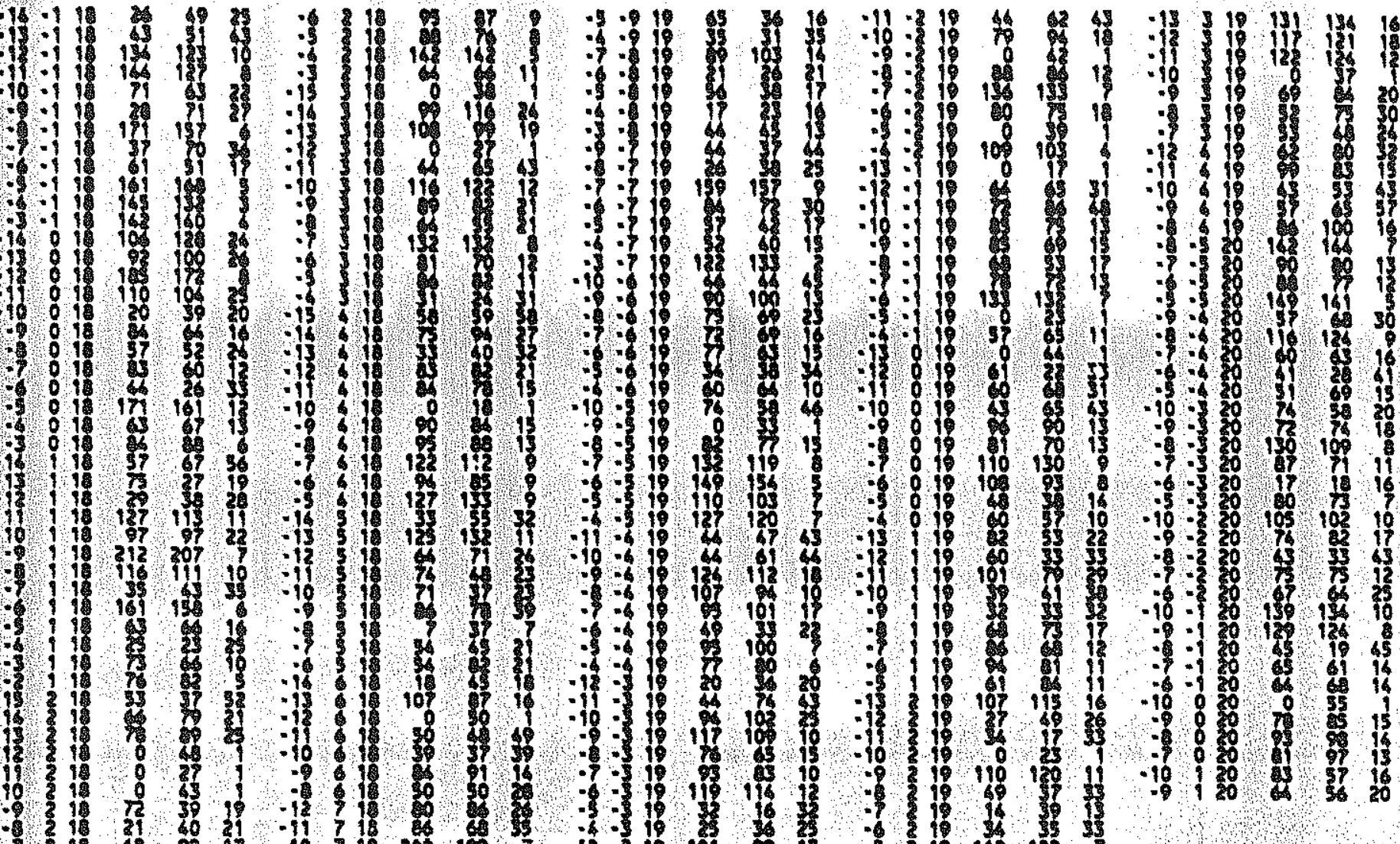

